Aus der Abteilung Kognitive Neurologie

(Prof. Dr. rer. nat. M. Wilke)

im Zentrum Neurologie

der Medizinischen Fakultät der Universität Göttingen

\title{
Assignment of thalamic nuclei using structural magnetization transfer MRI and orthogonal viewers
}

\author{
INAUGURAL - DISSERTATION \\ zur Erlangung des Doktorgrades \\ der Medizinischen Fakultät der \\ Georg-August-Universität zu Göttingen \\ vorgelegt von \\ Tabea Gringel, \\ geb. Witzkewitz \\ aus \\ Wissembourg/Frankreich
}

Göttingen 2012 
Dekan: Prof. Dr. med. M. P. Schön

I. Berichterstatter: PD Dr. rer. nat. G. Helms

II. Berichterstatterin: Prof. Dr. rer. nat. E. Irle

Tag der mündlichen Prüfung: 26. September 2012 


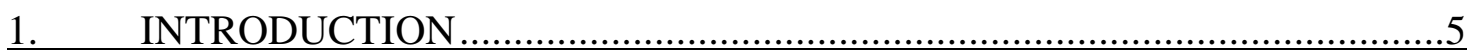

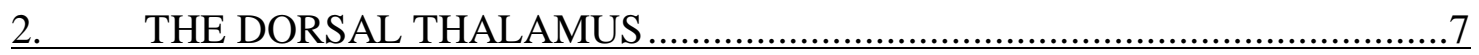

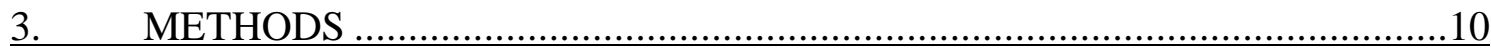

3.1. Structural MRI Contrast .................................................................. 10

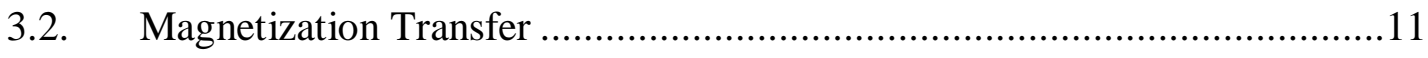

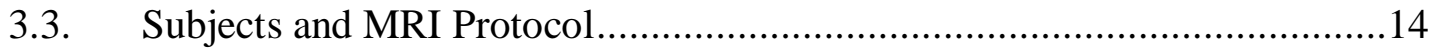

3.3.1. Post Processing of Images ......................................................... 15

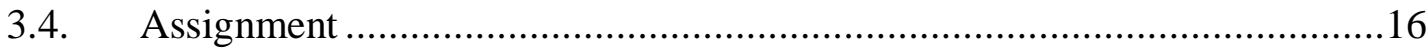

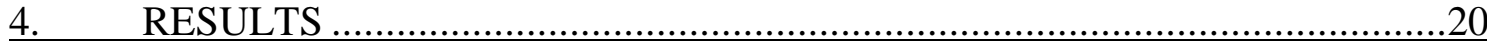

4.1. Thalamic Borders and Adjacent Structures .........................................21

4.2. Internal Medullary Lamina and Mamillo-thalamic Tract...........................22

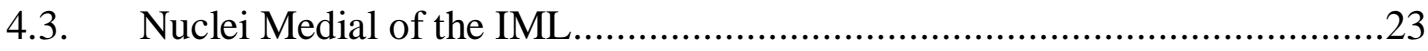

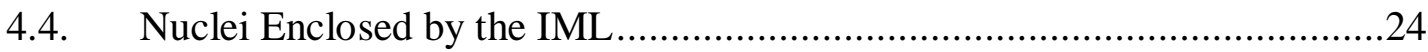

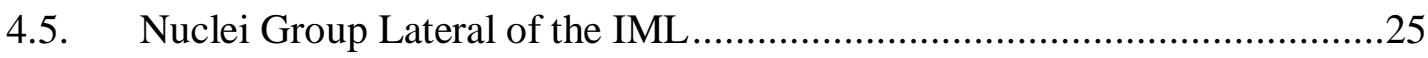

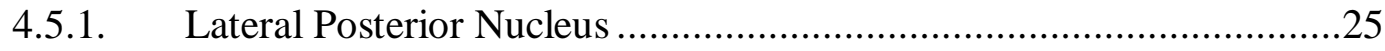

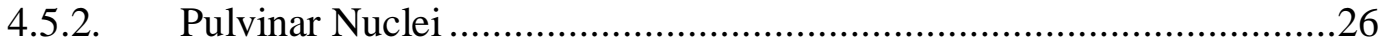

4.5.3. Geniculate Nuclei and Posterior Complex …..................................27

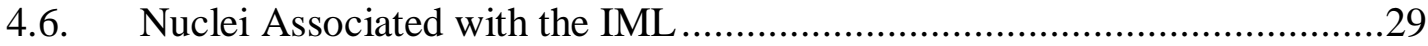

4.6.1. Anterior Group of Intralaminar Nuclei ..........................................29

4.6.2. Posterior Group of Intralaminar Nuclei ..........................................30

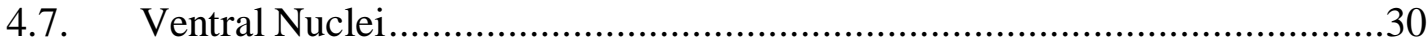

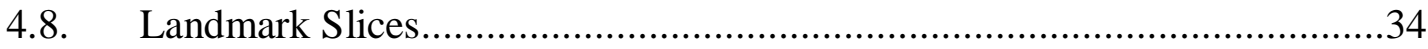




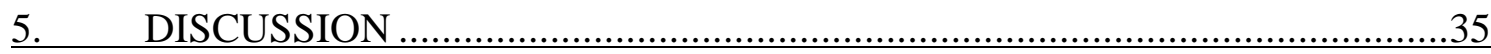

5.1. Intercommissural Reference System and Synoptic Orthogonal Viewing .....35

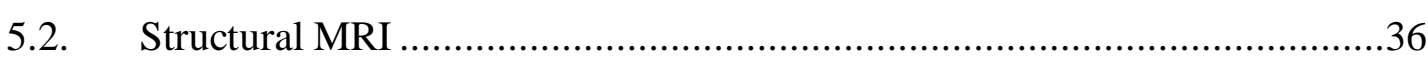

5.3. MT Maps, Classical and Population Atlases ................................................37

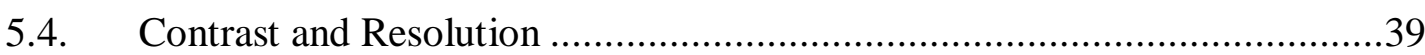

5.5. Potential Benefits for Stereotaxic Applications ............................................40

5.6. Challenges and Limitations ..................................................................42

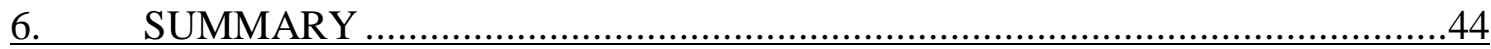

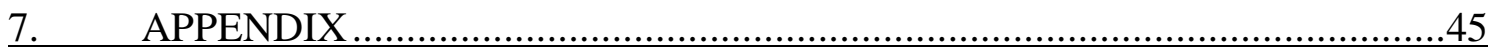

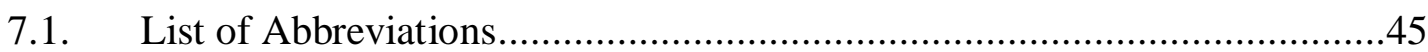

7.2. The Dorsal Thalamus Nuclei on MT Maps ………………………...........4

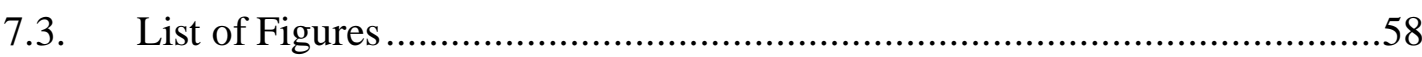

7.4. Publications related to this Dissertation ....................................................5

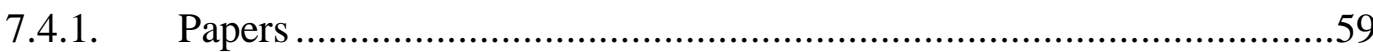

7.4.2. Presentations / Peer Reviewed Abstracts ...............................................59

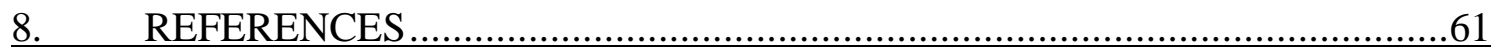




\section{INTRODUCTION}

"Therefore, it seemed desirable to work out the human thalamus in detail so that knowledge of the thalamus may be more complete."

Toncray and Krieg (1946, p. 423)

The Latin "thalamus" originates from the Greek thalamos ( $\theta \alpha \dot{\lambda} \alpha \mu \mathrm{o}$, Pschyrembel 2012, p. 2070) and refers to an inner room of a house as well as a bridal chamber. The use of "thalamus" in anatomical descriptions can be dated back to the second century AD to the physician Galen of Pergamon. However it is not clear if he referred to the diencephalic structure we know it to be today (Jones 2007). For sake of simplicity, "thalamus" will refer to the "dorsal thalamus", which is the subject of this thesis.

The nuclei of the thalamus were first described from post mortem dissections by macroscopic and later by microscopic observations. The development of magnetic resonance imaging (MRI) for neurominaging allowed individual human brain anatomy be depicted non-invasively. T1w(eighted) and T2-w structural MRI provides high contrast between cortical grey matter (GM) and white matter (WM). The thalamus, however, is notorious for its poor intrinsic contrast. This is due to the high axonal density in parts of the thalamus and the limited resolution of MRI in relation to the anatomical structures. Recent progress in high-resolution quantitative MRI has become instrumental for displaying (Deoni et al. 2005) and segmenting nuclei (Deoni et al. 2007) by means of the local relaxation times. MRI is also used for the construction of threedimensional (3D) digital atlases from two-dimensional (2D) histological sections (Niemann et al. 2000; Yelnik et al. 2007; Krauth et al. 2010). Diffusion tensor imaging (DTI) specifically targets the main axonal direction and coherence (Wiegell et al. 2003) but is more limited in resolution. Tracking the axonal connections with different cortical areas establishes a functional segmentation (Behrens et al. 2003; Jbabdi et al. 2009; Klein et al. 2010).

The excellent intrathalamic contrast obtained with a novel quantitative MRI technique for magnetization transfer (MT) contrast in the human brain at 3 Tesla (Helms et al. 2008c) encouraged the research that led to this thesis. The primary objective was to optimize a MRI protocol for multi-parametric mapping for application to the human thalamus at 3 Tesla (T). Acquisition and averaging of multiple gradient echoes showed considerable improvement of spatial resolution and signal-to-noise ratio (SNR) to resolve even smaller nuclei and to discern 
even smaller intensity differences. With this optimized protocol a cohort of healthy volunteers was examined on a conventional clinical MRI system. The possibility to depict the ironcontaining nuclei of the extrapyramidal motor system (Spatz 1922) turned up as an unexpected benefit of the technique (Elolf et al. 2007), although it is only lightly touched upon in this thesis. As a result, the assignment of thalamic substructures on the 3D "MT maps" became the scope of this thesis and was achieved by visual comparison to published stereotaxic atlases. This thesis therefore deals with problems pertaining to display, assignment, nomenclature and the choice of a reference coordinate system. Special attention was given to established targets for deep brain stimulation (DBS) like Hasslers's V.im (nucleus ventro-intermedius) and the subthalamic nucleus (STN).

In the first section a general outline of the function, anatomy and nomenclature of the dorsal thalamus will be presented. Basics of structural MRI contrast are explained as they are necessary for further understanding and is followed by a description of the study protocol (for details see Gringel et al. 2009).

The core part of this thesis is devoted to the assignment of thalamic nuclei using a synopsis of orthogonal views (Figure 11- Figure 39, p. 47ff.). A step-by-step approach is presented to locate the nuclei of the human dorsal thalamus. The main figures, which show the thalamic nuclei on the MT maps in different orientations, are compiled in an appendix for easier and faster access while reading the results.

Parts of this thesis have been presented in preliminary form at national and international workshops and conferences (listed in 7.4.2, p. 59f.). 


\section{The Dorsal Thalamus}

"However, we suggest that cerebral cortex without thalamus is rather like a great church organ without an organist: fascinating, but useless."

Sherman and Guillery (2001, p. 272)

The thalamus is an aggregation of neurons in the centre of the brain (Bähr et al. 2003) and one of the most complex structures in the central nervous system. The most prominent reference textbook devotes two detailed volumes exclusively focusing on all aspects of the thalamus and has recently been published in a second edition ("The Thalamus" by Jones 2007). Nearly all information of the periphery nervous system, including the environmental information and the intrinsic body receptors, is relayed in the thalamus. Thus, the thalamus takes the role of a mediator between the subcortical structures and the cerebral cortex and is involved in multitudes of pathways with feedforward, feedback, linkage, integration functions, attention and cognitive processes (Kandel et al. 2000; Sherman and Guillery 2001; Bähr et al. 2003; Guillery 2005; Sherman 2005; Jones 2007; Sherman 2007; Briggs and Usrey 2008; Wilke et al. 2010). "The dorsal thalamus, thus, gains entrée to virtually the whole cerebral hemisphere." (Jones 2007, p. 94). The olfactory system, however, is an exception as it bypasses the thalamus and directly moves on to cortical levels (Martin 1996; Kandel et al. 2000; Sherman 2005).

The dorsal thalamus has to be distinguished from the ventral thalamus (reticular nucleus, zona incerta, nucleus of the $(\mathrm{H})$ field of Forel; Jones 2007). The later is not to be confound with the ventral nuclei of the thalamus.

Contrary to the cortical organization in cytoarchitecturally and partially functional specific areas (Brodmann's areas), the thalamus forms an ensemble of nuclei. These are characterized functionally by their afferent and efferent connectivities projecting onto certain cerebral cortex areas (Jones 2007). In histology, a thalamus nucleus is identified by its cytoarchitecture, which is a cluster of neurons showing similar shape, size and density. Although recent immunohistochemistry insight did not unequivocally support the notion of nuclear entities, the use of this concept persists at different organizational and functional levels (Jones 2007). Both concepts have been adopted for neuroimaging studies.

Different nomenclatures of the thalamus in their specific labelling of the nuclei have been established by histological studies and each reflects the technology and knowledge of a certain 
era. Burdach (1819) for example examined slices of alcohol-hardened human brains with a magnifying lens and was the first to identify nuclear subdivision in the thalamus (Jones 2007). Among the scientists that devoted their research to the anatomy and function of the thalamus are Luys (1865), Nissl (1913), Le Gros Clark (1932), Walker (1938), Olszewski (1952) and Hassler (1959, pp. 230-290). Each one added details or even found reasons for a specific anatomical nomenclature (van Buren and Borke 1972; Jones 2007). Based on the history of the nomenclature by their former colleagues, Hirai and Jones delineated "a new parcellation of the human thalamus" (Hirai and Jones 1989, p. 1). Their aim was to unify the different nomenclatures existing for primates and non-primates. Furthermore, they emphasized the necessity for a common nomenclature in translation of findings on humans, other primates and non-primates.

Synonymous terms are tabled in the "Atlas for stereotaxy of the human brain" (Schaltenbrand and Wahren 1977) for the nomenclatures of Hassler (1959, pp. 230-290; Schaltenbrand and Wahren 1977), van Buren and Borke (1972), C. and O. Vogt (1941) and Walker (1938). Another synopsis of the nomenclatures of Hassler for humans (1959, pp. 230-290), as well as of Jones (1985), and Olszewski (1952) for non-human primates is given in the work of Hirai and Jones (1989).

In this thesis the nomenclature with the spelling of Hirai and Jones (1989) is used for the nuclei of the thalamus including the mamillo-thalamic tract and the internal medullary lamina. Tracts of WM are named according to the Schaltenbrand and Wahren atlas (1977) and the non thalamic structures are named according to North American terminology. 


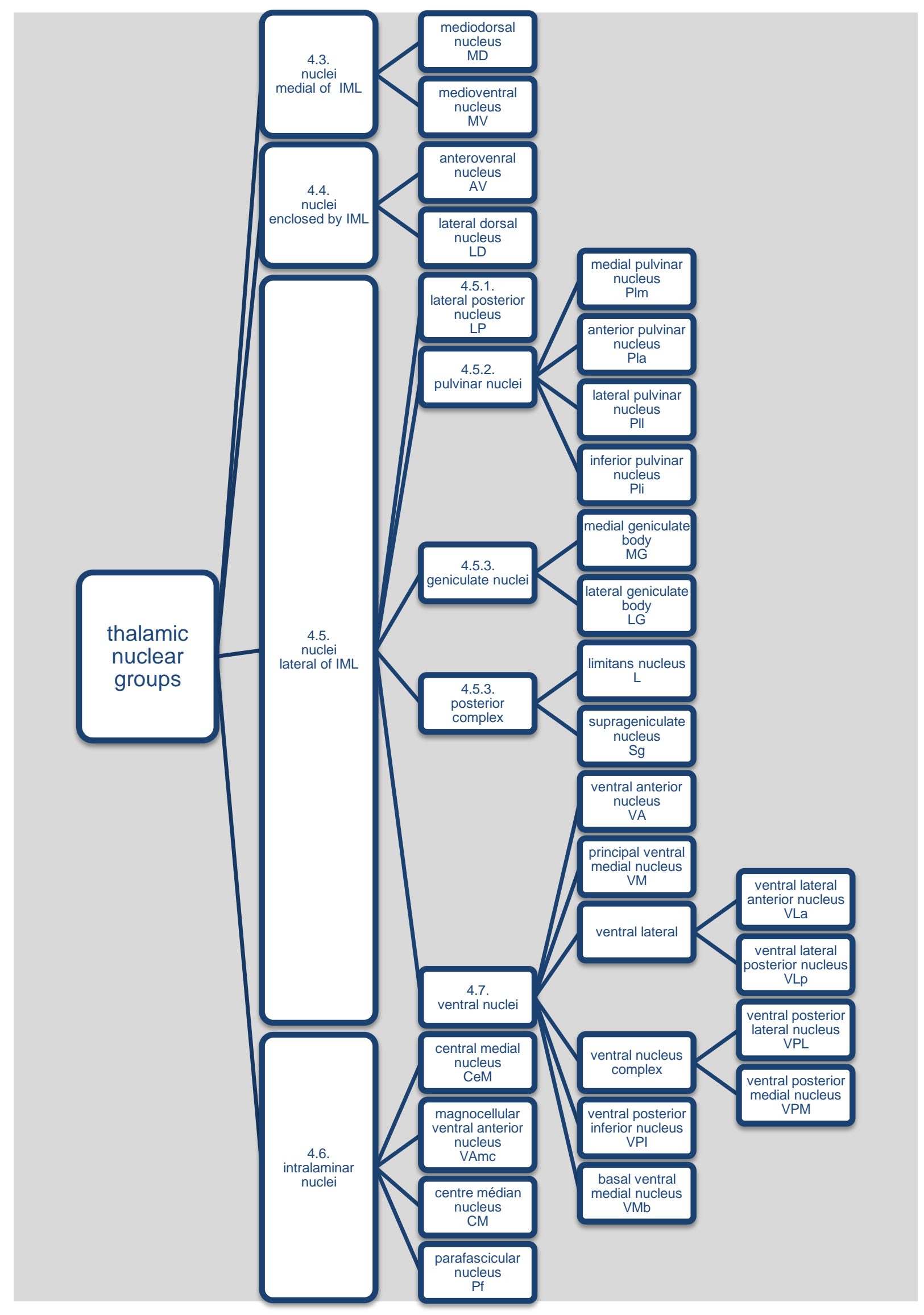

Figure 1: Chart with the classification by Hirai and Jones (1989)

25 nuclei listed that were identified on the MT parameter maps.

Numbers indicate the chapter in this thesis. 


\section{METHODS}

"May MT in MRI have an equally exciting second decade!"

Henkelman et al. (2001, p. 63)

\subsection{Structural MRI CONTRAST}

The most abundant element in organisms is hydrogen $(1 \mathrm{H})$ as part of water and organic molecules. MRI utilizes the large magnetic moment of the hydrogen nucleus in water (often too simply referred to as spin or proton). The contrast is influenced by the relaxation properties of the magnetization that is formed by the magnetic moments in a strong static magnetic field, usually 1.5 or $3 \mathrm{~T}$. In a general sense, "structural” MRI renders anatomical information, whereas “functional” MRI records “dynamic physiological information” (Symms et al. 2004, p. 1235).

Structural MRI contrast is created by relaxation properties of protons bound in large immobile molecules which are different from those of "free" water (Edzes and Samulski 1977). Only the mobile protons have a sufficiently slow transverse (or T2) relaxation to contribute to the MRI signal, whereas the so called bound protons in macromolecules relax within microseconds. Since this signal decay is too rapid to contribute to the MRI signal (Henkelman et al. 2001) macromolecules are MRI "invisible" and do not contribute to the observed proton density (PD) of the tissue. In the context of MRI, proteins, DNA (Deoxyribonucleic acid) and phospholipid membranes are collectively referred to as "structural material". However, they contribute to the observed relaxation of the mobile water because hydrogen atoms can be exchanged between the two pools (exchange) or two close spins can simultaneously change their orientation (crossrelaxation). In equilibrium, the same amount of magnetization is transferred from mobile water to macromolecules and vice versa. Thus, the net transfer is zero and does not change the size of the free water magnetization, even though it is much faster than the relaxation. Roughly, the higher the content of structural material, the more the relaxation times (T1, T2 and T2*) of the mobile water protons are shortened. The myelin coating of axons, the main part of white matter, is particularly rich in macromolecules and thus the dominant source of structural MRI contrast in brain. The sheaths of myelin provide a spatially tight arrangement of macromolecules and "myelin water". The second source of MRI contrast is paramagnetic ions, especially iron. They reduce T2 more strongly than T1 (and T2* even more so, as used in Elolf et al. 2007). Therefore, 
T1-w MRI is commonly referred to structural MRI, to a degree that these terms can be regarded as synonymous.

\subsection{MAgnetization Transfer}

Magnetization transfer contrast, or short MT, was first described in 1989 by Wolff and Balaban (1989). It selectively exploits the exchange of free water and macromolecules. This special MR contrast is evoked by irradiation in form of periodic radio frequency (RF)-pulses to selectively excite the bound protons and saturate (that is, reduce) their magnetization. Then, a net transfer of magnetization from the mobile to the invisible bound protons does occur (Symms et al. 2004). The resulting decrease in longitudinal magnetization is then detected as a reduced MRI signal. MT can be quantified by a complex physical model that includes the macromolecular fraction as most relevant parameter (Henkelman et al. 2001).

In clinical applications, the MT is expressed by the MT ratio (MTR), the percentage decrease of the steady-state-signal (Dousset et al. 1992). The reference is a second MR image with identical parameters but without MT-pulse. The MTR is corrected for the local signal strength (in arbitrary units) that is due to local proton density and the spatial profile of the receive coil. Thus, the values can be compared between two identical examinations. Such images of physical properties which are derived from generic MR images are called "maps". Published MTR values from human brain are between $20 \%$ to $60 \%$. They depend on

- the MT-pulse;

- the MRT sequence;

- the T1 relaxation;

- the local flip angle (Ropele et al. 2005; Helms et al. 2010).

Hence, the MTR is called a "semiquantitative" measure of MT.

A high fraction of bound spins, $F_{\mathrm{B}}$, induces an accelerated relaxation and is linearly correlated to the PD (Kamman et al. 1988). Thus, the larger the reduction of the steady state by MT is, the more it is counteracted by (faster) T1. This reduces the dynamic range of the MTR maps and the contrast between different tissue types.

An improved physical concept for MT takes into account that MT and T1 relaxation occur simultaneously in tissues under the conditions of fast exchange (Helms and Piringer 2005; Helms 2006). The free and bound spins no longer relax with their individual rate constants (the inverse of the relaxation times) but at a common average rate. After an imbalance of exchange between the two pools has been created by the MT-pulse, this imbalance is leveled by MT, while both 
pools relax back to equilibrium. The reduction of free water magnetization can be corrected for ongoing T1 relaxation. It evolves with a time constant of about $25-30 \mathrm{~ms}$ in brain tissue, which is much faster than the T1 relaxation (reflecting fast exchange; Helms and Piringer 2005). This percentage reduction following a single MT-pulse was subsequently termed "MT saturation" (Helms et al. 2008c). The MT saturation $\left(\delta_{\mathrm{MT}}\right)$ is a novel parameter that has been introduced to describe the MT contrast in MR pulse sequences. It is defined as the reduction of the free longitudinal magnetization corrected for common T1 relaxation. Under idealized conditions (no direct effect of the irradiation on free protons, complete saturation of bound spins, an equilibrium state) the theoretical maximum of the MT saturation is presented by the fraction of the bound spins.

If direct saturation (> $2 \mathrm{kHz}$ off-resonance) is avoided, the MT saturation is proportional to $F_{\mathrm{B}}$ but depends still on the strength and frequency of the MT-pulses (Helms and Piringer 2005). Like the MTR, the MT saturation is a semiquantitative parameter, but no longer influenced by T1. The characteristic values of the MT saturation are in the range of a few percent (unlike the much larger MTR values) but exhibits an improved contrast between GM and WM compared to MTR. A low $F_{\mathrm{B}}$ induces a small MT saturation and therefore an enhancement of the steady-statesignal but as well it induces an increase of the T1 and therefore to a lower steady-state-signal. To some extent these effects compensate each other, showing a poor contrast between white and grey matter. The histograms reflect this effect (Figure 2).

To calculate the MT saturation from spoiled gradient echo MRI (or FLASH, fast low angle shot) two additional acquisitions are necessary to determine the PD signal amplitude and the T1 relaxation. A simple calculation of the MT saturation is possible for 3D FLASH (Helms et al. 2008c) with non-selective excitation pulses. The use of 3D FLASH has some advantages: 1) FLASH has a simple signal equation that can be further approximated for a small flip angle and a short TR (Helms et al. 2008b); 2) a high isotropic resolution can be achieved; 3) the calculation of MT saturation out of three FLASH images will also cancel the spatial differences of the flip angle and not only that of the local coil sensitivity. Thus, spatially homogeneous maps of MT saturation are obtained without the need of additional RF mapping. Finally, the MT saturation represents "pure" structural contrast because it is inherently corrected for underlying variations in PD, T1, T2*, especially those induced by iron, which reduce the T1-contrast between deep brain nuclei and white matter (Helms et al. 2009a). 
In summary, the contrast of MT saturation maps resembles the contrast of T1-weighted images, with considerable advantages compared to conventional MRI:

- reproducible semiquantitative values independent of the local flip angle and the local coil sensitivity;

- high contrast between grey and white matter brain structures;

- high intrinsic contrast in deep brain grey matter structures such as in the thalamus (Gringel et al. 2009) but also in the basal ganglia (Helms et al. 2009a);

- $\quad$ independent of T1 and T2* and thus of iron in some deep brain nuclei;

- largely independent of the magnetic field strength (preliminary observations);

- independent of temperature (e.g. post mortem MRI).

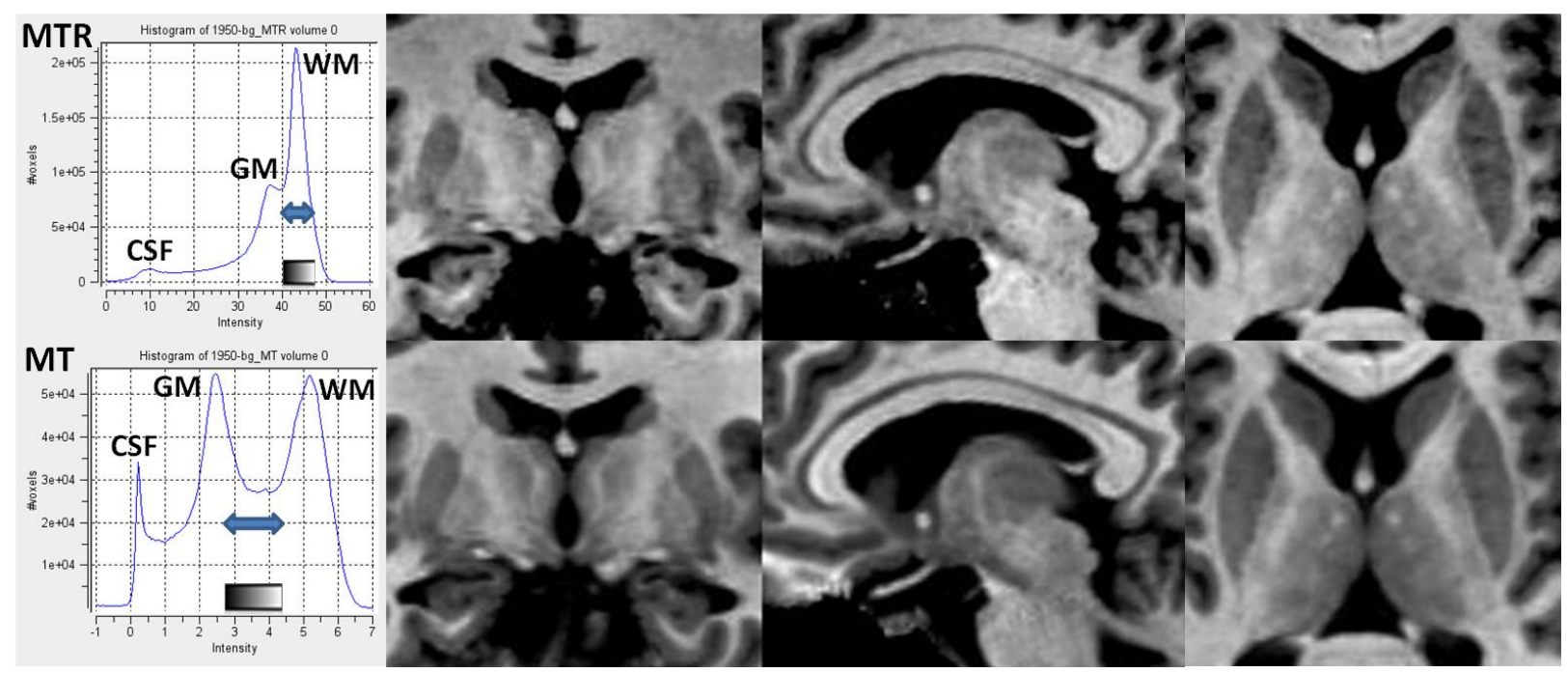

Figure 2: Histograms of MTR and MT

Top row: MTR values of the thalamus at $40-47 \%$. The intensity differences between the thalamic hemispheres are due to B1 inhomogeneity.

Bottom row: MT values of the thalamus between the peaks of grey and white matter at $2.75-4.25 \%$. Arrows in the histograms indicate the range of values of the thalamus.

Orientation of the panels from left to right: frontal, sagittal, horizontal. 


\section{3. $\quad$ SubJeCtS AND MRI PROTOCOL}

Twenty healthy adult subjects (aged 23 to 31 years mean 26.35 years, 10 males) participated in the study after giving informed written consent as supervised by the local ethical committee. MRI was performed on a $3 \mathrm{~T}$ whole-body MR system (Magnetom TRIO, Siemens Medical Solutions, Erlangen, Germany) using the body coil for transmission and the eight-channel phased-array head coil (Invivo, Gainesville, Florida USA) for signal reception. Details of the protocol optimization are given in Gringel et al. (2009).

Three sets of gradient echo images were acquired at $0.95 \mathrm{~mm}$ isotropic resolution $(0.856$ microlitre voxel size). A sagittal 3D-slab of $243 \mathrm{~mm}$ field-of-view was angulated along the midline of the brain (non-selective excitation of 176 sagittal partitions, 6/8 partial Fourier sampling and partial parallel acquisition using GRAPPA (generalized autocalibration procedure for partial acquisition, factor 2, 24 reference lines). The SNR was increased by averaging 8 multi-echo acquisitions at echo times $\mathrm{TE}=2.3,5.3, \ldots 23.3 \mathrm{~ms}$ with a bandwidth of $370 \mathrm{~Hz}$-perpixel (Helms and Dechent 2009).

By choice of flip angle (FA) and repetition time (TR) each set provided a different contrast of i) predominantly MT- weighting ( $\mathrm{TR}=48 \mathrm{~ms}, \mathrm{FA}=10$ degree, gaussian-shaped MT-prepulse of $9960 \mathrm{~ms}$ duration, 500 degree nominal flip angle and $1200 \mathrm{kHz}$ resonance offset); ii) T1weighting ( $\mathrm{TR}=30 \mathrm{~ms}, \mathrm{FA}=20$ degree$)$, and iii) proton density $(\mathrm{PD})$ weighting $(\mathrm{TR}=30 \mathrm{~ms}$, $\mathrm{FA}=7$ degree). Total measuring time was 25 minutes.

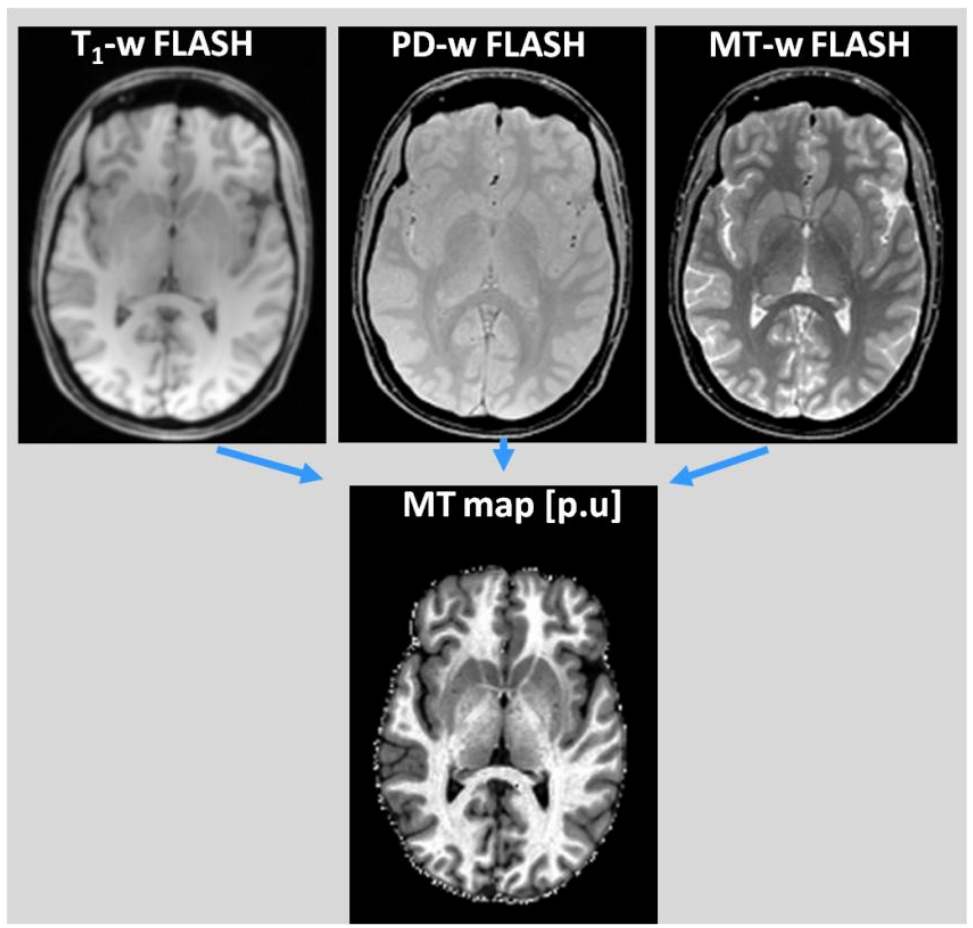

Figure 3: Calculation of MT saturation maps from MT-w, PD-w and T1-w FLASH images

Modified from Helms et al. (2007, Poster \# 2057) 


\subsubsection{Post Processing of Images}

Parameter maps of T1, signal amplitude, and MT saturation (i.e. the percentage of longitudinal magnetization transferred after a single MT-pulse/during one TR) were calculated using the routines of the FMRIB software library (FSL 3.2, Functional Magnetic Resonance Centre of the Brain, University of Oxford, www.fmrib.ox.ac.uk/fsl) as described in Helms et al. (2008c). The assignment of the thalamus nuclei was based on the MT saturation because these provided the highest contrast within the thalamus and to the surrounding structures (Gringel et al. 2009).

The reference coordinate system chosen in this thesis is based on the line joining the centres of the anterior and posterior commissures. The anterior commissure (AC) is shown in Figure 11 sagittal view, Figure 13 axial panel P, Figure 14 sagittal panel R; and the posterior commissure (PC) is shown in Figure 12 coronal panel H, Figure 13 axial panel P, Figure 14 sagittal panel R. Each MT map was aligned onto a modified T1-weighted template of the deep brain region including the basal ganglia and the corpus callosum to achieve a reproducible angulation along the intercommissural (AC-PC) line. The template was constructed from the MNI152_T1_0.5mm brain template provided with FSL 3.2 (FMRIB's Linear Image Registration Tool (FLIRT); http://www.fmrib.ox.ac.uk/fsl/; correlation ratio, 6 Degrees of Freedom, angulated by 7 degree, see Gringel et al. 2009). The selection of only the thalamus from the whole brain was less reliable, probably because the inferior and lateral thalamic borders do not provide highly contrasted contours. The thalamus was therefore cropped at the corpus callosum and the midbrain. The radiological right-left convention was maintained in the volume data.

The angulated and upsampled volume data was displayed using the MRIcro viewer (www.sph.sc.edu/comd/rorden/mricro.html).

In the following, the term "position" will be reserved for the coordinate along the intercommissural line, which is most important for the spatial assignment. "Level" will refer to the horizontal planes.

During development the brain axis bends towards anterior, so that the (initially) ventral aspect of the thalamus faces towards inferior and the dorsal aspect towards superior.

The terms "horizontal" and "axial", "frontal" and "coronal", "superior" and "dorsal", "ventral" and "inferior", "rostral" and "anterior" are used synonymously. "Hyper"- and "hypointense" refer to local contrast. 


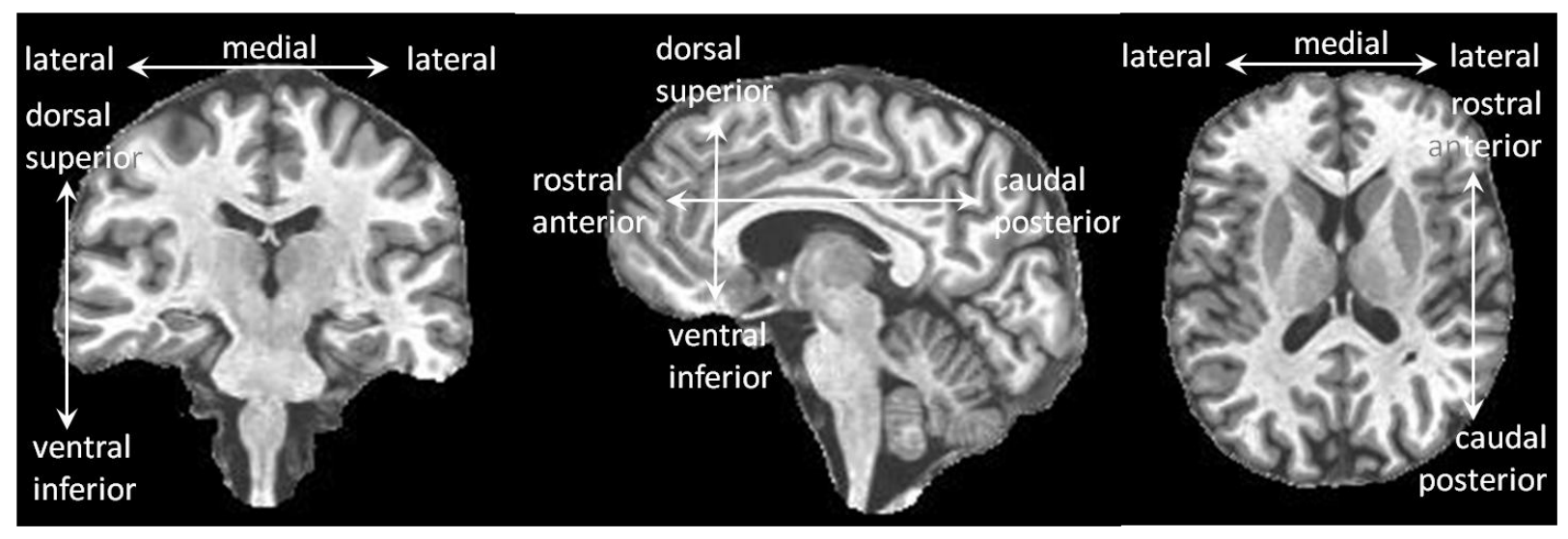

Figure 4: Anatomic labelling

From left to right: coronal, sagittal and horizontal view (labeling modified from Kandel et al. 2000, p. 321). Whole brain MT map from subject hum_1950.

\subsection{ASSIGNMENT}

The assignment of the nuclei was initially achieved by browsing through the 3D MT maps of a single subject and comparing it with the literature (Figure 5; Schaltenbrand and Wahren 1977; Hirai and Jones 1989; Morel et al. 1997; Jones 2007; Gallay et al. 2008; Mai et al. 2008). In this process, major landmarks were identified that facilitate the orientation on the MRI images. The window settings were empirically optimized (centre=4, width=6.5) and consistently applied in all subjects. The assignment was then validated by placing opaque contour plots constructed from the camera lucida drawings in Jones (2007) onto the corresponding slices (Figure 6 and Figure 7) and finally cross-checked on the whole cohort. Frequencies are given when a structure could not be consistently discerned in all subjects.

The myelin staining featured in the atlases of Schaltenbrand and Wahren (1977), Mai et al. (2008) and Gallay et al, (2008) is closely related to the mechanism of MT contrast. Nevertheless, the acetylcholinesterase staining of Hirai and Jones (1989) corresponded well to the structural contrast (Figure 5), as well as the parvalbumin staining of Morel et al. (1997; not shown). The thionin staining in Hirai and Jones (1989) and calbindin D-28K and calretin staining in Morel et al. (1997) showed somewhat less correspondence to the data. 


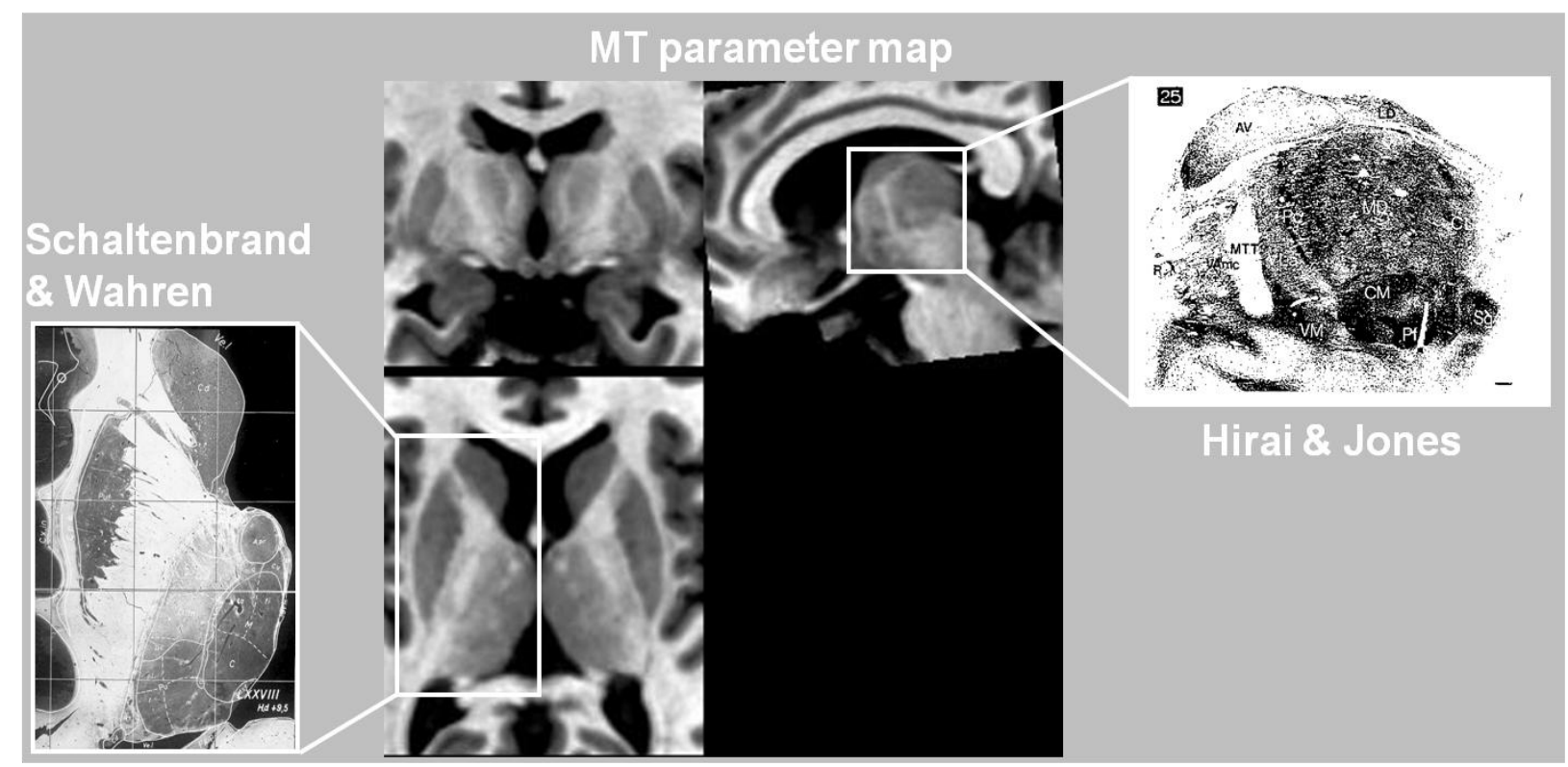

Figure 5: Comparing MT maps to selected atlases

Schaltenbrand and Wahren (1977, plate 51, LXXVIII H.d +9.5): myelin staining -inverted Hirai and Jones (1989, p. 20, Figure 25): acetylcholinesterase staining

MT parameter map (hum_1950-65-89-88): horizontal view was tilted $7^{\circ}$ towards anterior to match the Schaltenbrand and Wahren atlas.

Three intuitive criteria were employed for the assignment of thalamic substructures: contrast differences to adjacent structures, delimiting laminae of WM and relative positions. For the latter, a synopsis of three orthogonal views is particularly helpful. After a nucleus has been located on one view, landmarks can be identified (or cross-checked) on the other views. The following description of each nucleus will thus proceed from the most to the least conspicuous views, though exceptions are made for the sake of brevity and readability.

The more easily identifiable structures served as landmarks for tracing the minor nuclei. It was helpful to start with the medial nuclei, and then precede via the anterior group along the lateral ventricle to the pulvinar nuclei. The inferior structures and the IML are also relatively easy to identify and served as landmarks to assess the ventral nuclei. These proved most challenging and thus treated last. The extent of the red nucleus (RN) and the subthalamic nucleus (STN) can serve as additional landmarks. Like on T1-w MRI, the RN and STN are poorly distinguished from surrounding WM and thus cannot be delineated reliably on the MT maps. These nuclei were identified by their high effective transverse relaxation (Elolf et al. 2007) on an opaque colour overlay of the R2* maps (Helms et al. 2008a) as seen on Figure 6 and Figure 7. 


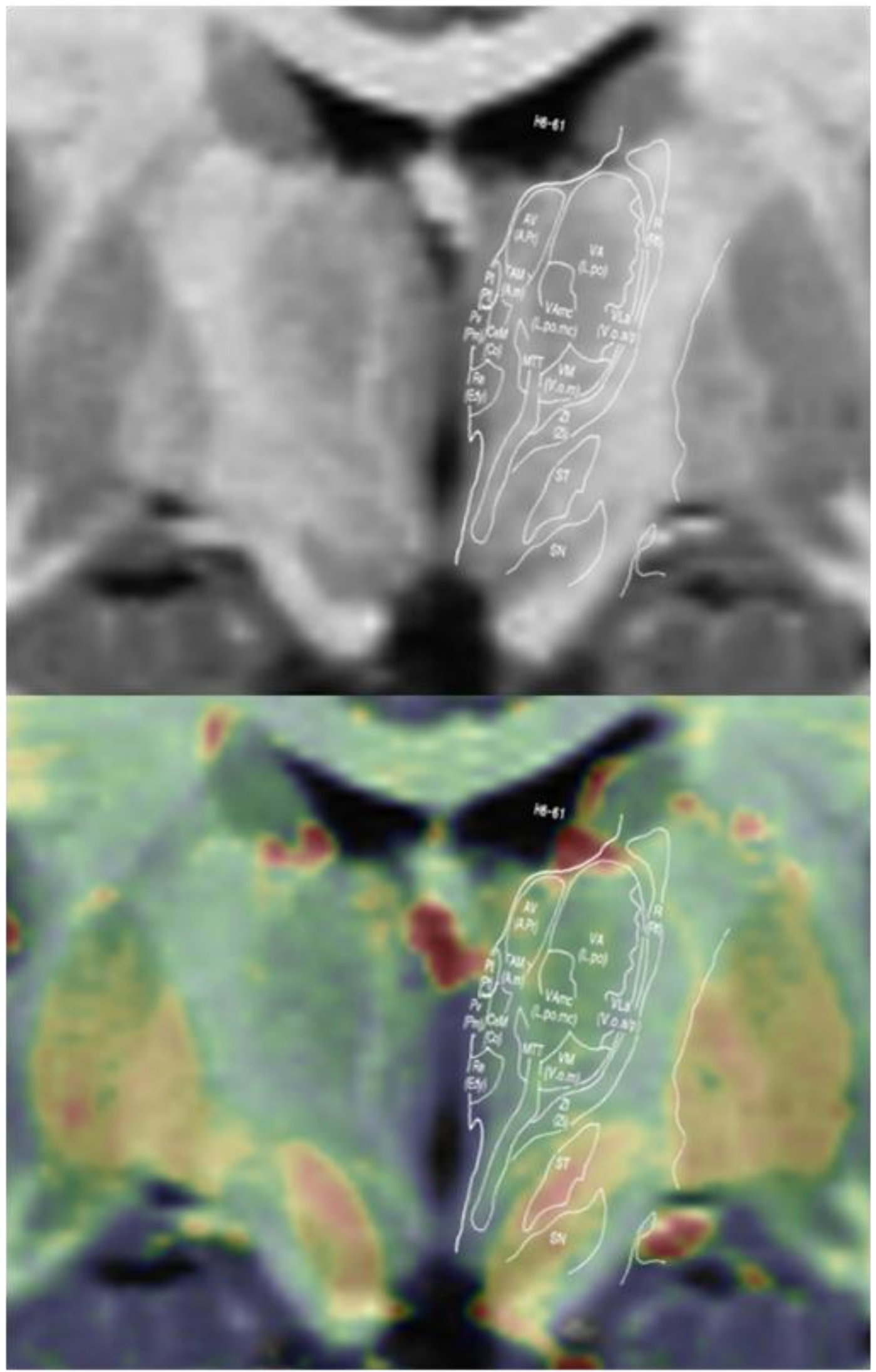

Figure 6: Overlay of camera lucida drawings on MT and R2* maps - coronal

For details see legend of Figure 7 


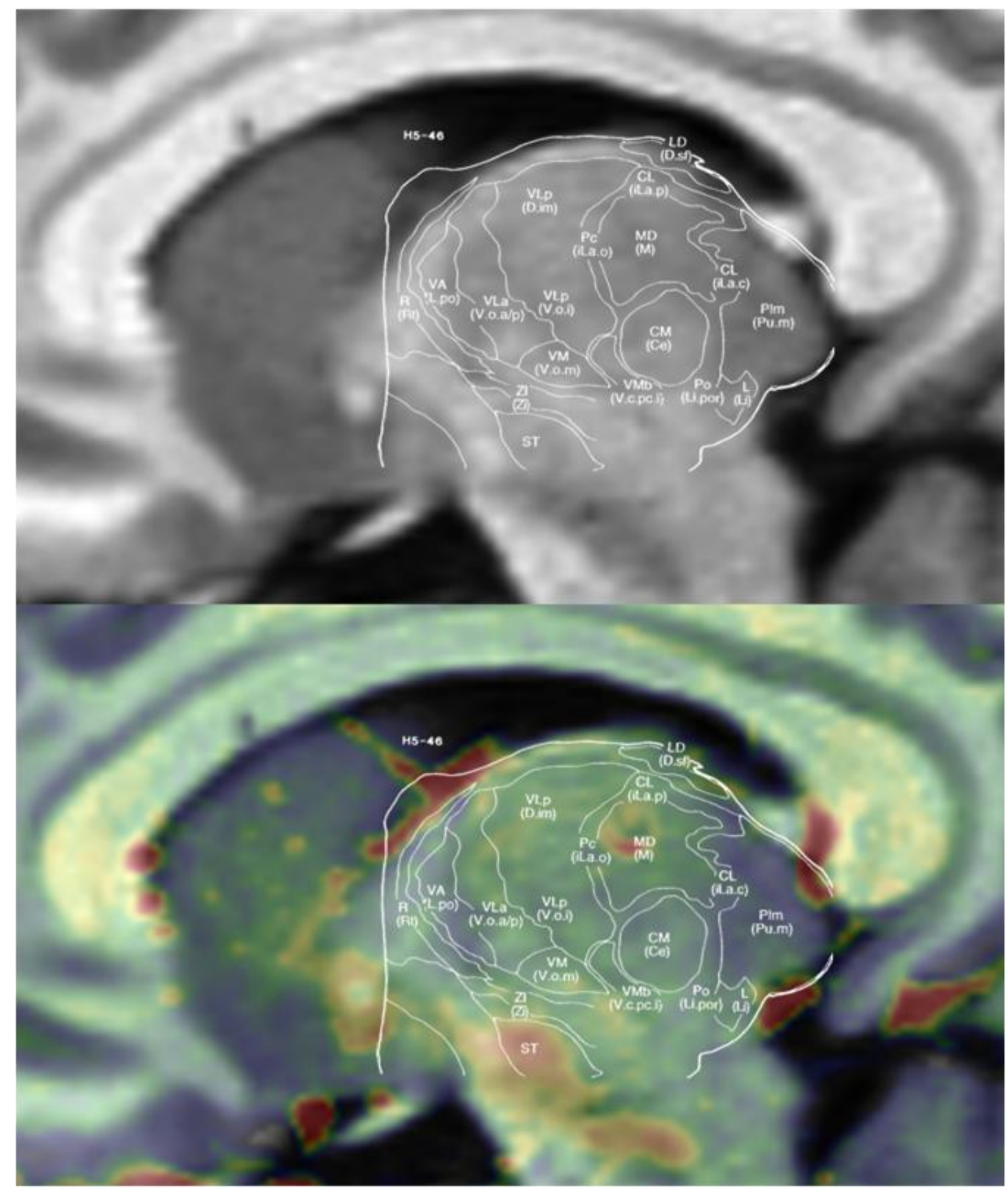

Figure 7: Overlay of camera lucida drawings on MT and R2* maps - sagittal

Overlay of camera lucida drawings from Jones (2007): coronal H6-61, sagittal H5-46 (Jones 2007, coronal p. 1431, sagittal p. 1434) and MT maps of hum_2386 (x92, y88).

Bottom row: MT map with additional colour overlay of R2*. The iron-rich substantia nigra is seen as a red band below the subthalamic nucleus (ST). 


\section{RESULTS}

"The thalamus is like the Flying Dutchman: many have heard of it, some believe in it, but few have actually seen it."

Rose cited 1940 (quoted in Jones 2007, p. 43)

The MT saturation maps of the deep brain region resemble T1-weighted MR images, but with a strongly enhanced contrast and without a spatial intensity bias (Gringel et al. 2009). The typical higher intensity towards the internal capsule (IC) is seen in coronal and axial views (Figure 12, Figure 13). The axonal bundles that run into the IC and that can be seen with myelin staining (Mai et al. 2008), were not resolved at $0.95 \mathrm{~mm}$ resolution. The medial group of the thalamus and the pulvinar nuclei had the lowest intensity within the thalamus but were slightly brighter than cortical GM. On the other hand, the ventral nuclei appeared hyperintense but were darker than white matter. The intermediate MT values found in the thalamus likely represent varying fractions of perikaryontic cell bodies and myelinated axons. This may also explain why a number of larger nuclei (e.g. the mediodorsal nucleus (MD), the ventral lateral posterior nucleus (VLp), the lateral pulvinar nucleus (Pll) all in Figure 13 horizontal panel L) did not show a homogeneous intensity. These spatial variations in intensity were consistent between subjects. Often there were no clear borders, so the relative positions of the nuclei are described rather than their extension or a ROI analysis of mean MT values.

In the appendices, Figure 12 to Figure 14 display a selection of continuous slices either in coronal (Figure 12), horizontal (Figure 13) or sagittal (Figure 14) views. In the following, these figures will be referred to as "panels" for the sake of brevity. Each identified nuclei is as well shown individually in an orthogonal view in Figure 15 to Figure 39 (see 7.2). Where no clear delineation in the MT maps was possible, the confluent nuclei were marked by a slash in the panels (e.g. as $\mathrm{VLa} / \mathrm{p}$ in sagittal panel T). 


\subsection{Thalamic Borders AND AdJACENT Structures}

This description is intended to provide landmarks for assignment, as well as to aid the manual delineation of the thalamic boundaries.

The lateral borders to the posterior limb of the IC are seen in axial slices. However, the reticular nucleus of the ventral thalamus and the external medullary lamina were not resolved by the 0.95 $\mathrm{mm}$ voxels. This leaves a residual uncertainty of the lateral thalamic boundary of about $1 \mathrm{~mm}$. This problem is exacerbated by the axonal tracts passing from the thalamus into the IC. The pyramidal tract (PT) is characterized by reduced MT values (marked in Figure 11; not marked in axial panels L-Q; sagittal panel V; marked in sagittal panel W) as seen also in standard T1-W and T2-w MRI (Yagishita et al. 1994). The reduced contrast towards the PT may hamper delineation in the corresponding coronal panels $\mathrm{E}, \mathrm{F}$.

The lateral and inferior borders of the thalamus are best seen in coronal views (coronal panels AI). The inferior border is more complex than the lateral border because it is not formed by a single WM tract. It is difficult to distinguish at positions anterior to the red nucleus (coronal panels A-D). Nevertheless, the inferior border can be followed by browsing through continuous coronal views (coronal panels A-I) and referring to adjacent structures. Note that, the angle formed by the lateral and inferior borders is not constant, but decreases from anterior (135 degree, coronal panel B) to posterior (125 degree, coronal panel F).

Most anteriorly, at positions between the columns of the fornix and the mamillo-thalamic tract (MTT, see 4.2), the highly vascularized tissue of the hypothalamus (HT) can be distinguished by its typical hypointensity (Figure 11; coronal panel A, B; sagittal panel S).

In the coronal slice that displays the whole extent of the MTT (Figure 11; coronal panel C), wing-like oblique bands can be seen inferior of the thalamus: The most ventral of these is the hyperintense aspect of the cerebral peduncle followed dorsally by two hypointense bands, first by the substantia nigra ( $\mathrm{SN}$ ) and then by the hypothalamus. Superior to the RN, the thalamic border runs almost horizontally as seen clearly in the coronal views (coronal panels E, F; sagittal panel S). Between this position and the hypothalamus, the thalamic border has to be interpolated (coronal panel D). Here the zona incerta (of the ventral thalamus) cannot be seen in the MT maps. As in the case with the reticular nucleus this resulted in an uncertainty about the thalamic border.

Posterior of the RN, the thalamus does not exhibit a straight border which can be associated with a single WM tract (coronal panels G, H, I). In inferior axial views, well delineated nuclei (VPL, MG, LG, Pli see 4.5.2) protrude like "lobes" into the subthalamic region (axial panel Q). 
At the superior and anterior borders, the contrast to the lateral ventricle is enhanced by a tenuous hyperintense band of WM. It represents part of the stria medullaris (marked in sagittal panel S).

\subsection{InTERnAl MEdUllary LAMina AND MAMILlo-Thalamic Tract}

The internal medullary lamina (IML) and the mamillo-thalamic tract (MTT) are the discernable WM structures inside the thalamus. They are seen as continuous hyperintense structures in the MT maps and also in T1-weighted images. Hirai and Jones (1989) adopted the classification of the thalamic nuclei according to their spatial relation to the IML into groups lateral and medial from the IML and the anterior group being enclosed by the anterior branches of the IML. The lateral nuclei are further divided into the ventral nuclei, the pulvinar nuclei, the lateral posterior nucleus and the geniculate nuclei. The nuclei scattered within the IML are classified as the intralaminar group (Figure 1).

The starting point for orientation in the thalamus is usually the MTT. It can be easily identified in each view (Figure 11). In the AC-PC angulation, the whole extent of the MTT can be displayed in a single coronal slice (coronal panel C). In more than half $(11 / 20)$ of the subjects, however, the two MTT's were not exactly opposite to each other. Below the commissural level, the MTT appears as a hyperintense dot posterior of the hypothalamus (axial panels O-Q). From here it can be traced upwards through contiguous slices. The diameter of the MTT appears to increase as it cannot be differentiated from adjacent parts of the IML.

The anterior branches of the IML start at the superior end of the MTT (axial panels K, L; coronal panels B, C, D). The posterior branch of the IML is less distinct because it appears interrupted by the intralaminar nuclei (see 4.6). It is best seen in axial views (axial panels L-N). The intralaminar nuclei also explain why the IML does not consistently appear in different coronal views (coronal panels D-H). Nevertheless, the course of the IML can usually be interpolated across the gaps in axial and coronal views (as indicated by the dotted lines in Figure 11). "The dorsolateral part of the Lamina (IML) [...] is broken up into clustered aggregates that invade the medial nucleus of the pulvinar behind the mediodorsal nucleus" (Jones 2007, p. 1399). These "aggregates" could not be seen in coronal views, but are likely responsible for the inhomogeneous appearance of the medial pulvinar (see 4.5.2).

About $5 \mathrm{~mm}$ from the sagittal midline, the adjacent structures of MTT and IML can be seen as an oblique spur protruding from the midbrain anterior of the red nucleus (sagittal panel S and Fig. $6 \mathrm{~b}$ of Gringel et al. (2009)). This slice also features a hyperintense rim around the medial nuclei formed by the superior part of the IML, the MTT and the fasciculus retroflexus Meynertii (arrow 
in sagittal panel S). The MTT/IML constitutes the anterior border of the medial group. The short anterior branches of the IML are not seen in this slice.

\subsection{NUClei MEdial OF THE IML}

The large mediodorsal nucleus (MD, Figure 15) can always be identified (coronal panels D-H; axial panels L-N; sagittal panel S). At its widest cross-section, the MD takes up to two thirds of the anterior posterior extent and up to half of the medial to lateral extent of the thalamus (coronal panel F; sagittal panel S). The medioventral nucleus (MV, Figure 16) is found medial to the MTT at the interthalamic adhesion and expands ventrally along the border of the third ventricle (coronal panel C). In about $30 \%$ of humans, the MV nuclei are connected to form the nucleus reuniens (Jones 2007). This nucleus could not be verified in our healthy cohort, because the nucleus reuniens is only seen in the presence of atrophy against the surrounding cerebrospinal fluid (Figure 8). The parataenial nucleus is too small to be identified.

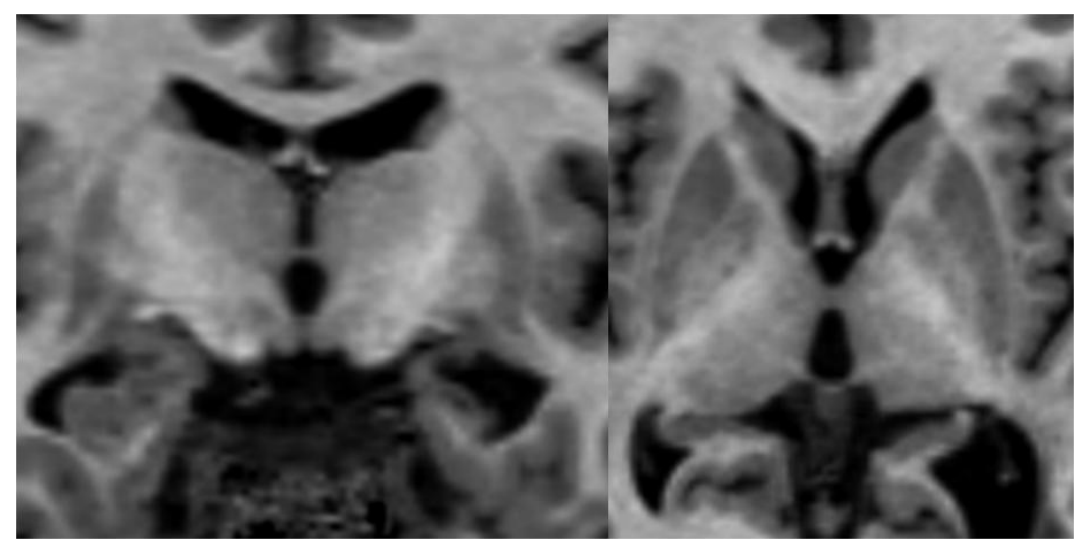

Figure 8: Nucleus reuniens

MT map of a patient (female, 52 years): nucleus reuniens clearly visible in the presence of atrophy at interthalamic adhesion.

left: coronal view; right: horizontal view.

The large MD is detected in each orientation situated medial of the IML and posterior to the MTT (axial panels L, M; sagittal panel S). In axial and coronal views, the nucleus displays an olive shape with the third ventricle forming its medial border (axial panels L-N; coronal panels D-H). In the sagittal view at the position of the MTT, the central round shape marks the crosssection of the MD (sagittal panel S). The MD is surrounded by the hyperintense IML laterally and dorsally (coronal panel D and sagittal panel S). At positions where the IML is poorly visible, the MD can still be discerned from the adjacent nuclei by its lower intensity (coronal panel F). 
Although most of the boundaries of the MD can be clearly delineated, its anterior and posterior poles are difficult to determine. The posterior pole lies slightly posterior to the habenular nucleus and blends into the medial pulvinar (axial panel $\mathrm{N}$ ). At the anteroinferior border, several small nuclei are found, which require a more detailed description (axial panels $\mathrm{M}, \mathrm{N}$; see MV/CeM below).

The MT maps show a slight intensity gradient from medial to lateral across the MD. This may be mainly explained by axonal density. Although histochemical staining provide evidence of subregions (named after their cytoarchitecture in magnocellular, parvocellular, multiform; Jones 2007), these could not be clearly assigned in the MT maps though the magnocellular part coincides with the most hypointense medial region. Addition image inhomogeneity is caused by the superior thalamic vein that passes the MD (Giudicelli and Salamon 1970). It appears slightly hypointense in the MT map, but can be more clearly identified by superposition of the R2* map (see sagittal view in Figure 7: vein as a red dot in the MD).

The smaller medial nuclei can only be identified by their position with the aid of the crosshair: Most conspicuous is - again - the coronal view containing the MTT (coronal panel C). The region medial of the MTT is filled by the MV and by the anterior portion of the central medial nucleus (CeM, of the intralaminar group see 4.6.1, Figure 28 and Figure 29). These two nuclei can only be located by their relative positions: the CeM lies slightly above the MV. In axial views the MV and CeM lie anterior of the MD (axial panels M, N). Localization in sagittal slices requires the use of the crosshair due to absence of clear landmarks and similar densities to the surrounding nuclei (sagittal panel R).

\subsection{NUCLEI ENCLOSED BY THE IML}

The three anterior nuclei and the lateral dorsal nucleus (LD, Figure 18) are embraced by the branches of the IML and border on the lateral ventricle.

The largest and most conspicuous of these, the anteroventral nucleus (AV, Figure 17) forms the anterior-superior pole of the thalamus (coronal panel A). This nucleus extends towards superior "despite its name" (Jones 2007, p. 1210; sagittal panel S). It is the only nucleus together with the ventral anterior nucleus (VA) seen in the most anterior section depending on the angulation and the individual anatomy (coronal panel A), though Figure 5 gives a different expression. The AV is well delineated by the anterior IML branches and the lateral ventricle. Thus, it can be identified in each view (coronal panels A-D; axial panels J-M; sagittal panel S). The AV is best seen in coronal and sagittal views showing the MTT (coronal panel C; sagittal panel S): Its cross-section is an oblique oval in the coronal view and scimitar-like in the sagittal 
view. The horizontal cross-section of the AV is obliquely oval at superior levels and diminishes in size towards the interthalamic adhesion (axial panels J-L).

The small anteromedial nucleus and anterodorsal nucleus escape identification: The anteromedial nucleus merges into the AV with probably the same intensity. Even in histological preparations these two nuclei "lack [...] a clear distinction" (Jones 2007, p. 1398) and the anterodorsal nucleus is probably disguised by partial volume averaging with the ventricle.

The lateral dorsal nucleus (LD, Figure 18) forms a thin tail on top of the most superior portion of the MD (coronal panel E; axial panel J; sagittal panel S). In sagittal and horizontal views it appears as a continuation of the AV. The LD borders medially on the ventricle and laterally on the IML branch (axial panel J). Thus, the position of LD appears to be more medial than lateral despite its name. Because there is no definite change in contrast, the two nuclei seem to undergo a continuous transition (sagittal panel S). If not affected by atrophy, differentiation between AV and $\mathrm{LD}$ is possible by the shape of the coronal cross-section: the LD is rather flat along the surface of the lateral ventricle (coronal panel E) whereas the AV is rather round (towards the position of the MTT; coronal panels B-D). The contrast between LD and IML decreases towards posterior (coronal panel E; sagittal panel S). The posterior end of the LD is usually disguised by partial volume effects. Its tip extended to positions posterior of the PC in 4/20 subjects.

\subsection{NUCLEI GROUP LATERAL OF THE IML}

The lateral group is further divided into the lateral posterior nucleus, the pulvinar nuclei, the geniculate nuclei and the ventral nuclei (for later see 4.7, p. 30ff.).

\subsubsection{Lateral Posterior Nucleus}

Together with the pulvinar nuclei, the lateral posterior nucleus (LP, Figure 19) forms the posterior portion of the thalamus (sagittal panel $U$ ). The anterior extension of the pulvinar and the LP can be reliably identified in axial and sagittal views by their lower MT values (axial panels K-P; sagittal panels T, U). This is in agreement with the low number of axonal fibres seen in myelin staining (Mai et al. 2008). In the coronal slices anterior of the PC (coronal panels G, $\mathrm{H})$, however, the LP shows poor contrast to the neighbouring nuclei and has to be assigned by its relative position between the MD and the ventral lateral posterior nucleus (VLp, see 4.7). In the coronal slice posterior to the PC the LP "comes to the dorsal surface of the thalamus and then ends by merging with the medial and lateral pulvinar nuclei” (Jones 2007, p. 1010; coronal panel 
I). In this panel, the LP extends towards the lateral ventricle and the IC and is delimited against the pulvinar by a diffuse hyperintense band (seen in 17/20 right thalamus, 16/20 left thalamus). In horizontal views, the LP is found at levels superior to the interthalamic adhesion (axial panels $\mathrm{K}-\mathrm{M}$ ), where the posterior part of the thalamus appears hypointense. This portion comprises the LP and merges antero-lateral into the VLp, posterior into the lateral pulvinar nucleus (PIl, see 4.5.2), and medial into the apex of the MD (axial panels L, M). In sagittal slices lateral to the MTT, the vertical extent of the whole posterior thalamus is seen as a hypointense area (sagittal panel T). The upper part of this hypointensity consists of the LP blending into the pulvinar without a clear line of demarcation. In the more lateral sagittal panel V the LP is more clearly outlined.

\subsubsection{Pulvinar NuClei}

The labelling of the four pulvinar nuclei (medial (Plm, Figure 21), anterior (Pla, Figure 20), lateral (Pll, Figure 19), and inferior (Pli, Figure 23)) is consistent with their anatomical location. This facilitates the assignment although the hypointense pulvinar nuclei are of similar intensity and lack laminar borders. The axial view is best suited to identify the pulvinar nuclei, because it is roughly orthogonal to the intra-pulvinar borders, which easily escape identification in coronal views. About levels halfway between the commissures and the interthalamic adhesion, the hypointense posterior portion of the thalamus can be unambiguously assigned to the pulvinar nuclei (axial panels $\mathrm{N}, \mathrm{O})$. It is useful to reduce the windowing width $(\mathrm{c}=4, \mathrm{w}=3.5)$ to enhance small intensity differences. The hypointensity is degraded in the anterior portion of the Plm, where IML and intralaminar nuclei extend into the pulvinar (see 4.2).

The Pla - as it name implies - bulges to the anterior between the hyperintense ventral nuclei (lateral, see 4.7) and the centre médian nucleus (CM, medial, see 4.6.2, p. 30, axial panel O). It is located anterior to the Plm and medial of the Pll. The Plm is found in the medial-posterior region next to the cisterna laminae tecti; the Pll in the latero-posterior region adjacent to the IC. This arrangement of nuclei could be followed towards lower levels (in 8/20 subjects); occasionally even down to the AC-PC level like in axial panel P. The axial cross-section of the Plm gets bigger towards the inferior. Simultaneously the Plm takes up a more posterior position and the hypointense limitans nucleus (L, Figure 26) appears medially, next to the habenular nucleus. The L can be, therefore, easily interpreted as part of the pulvinar, although it is part of the posterior complex (see 4.5.3). At this level (axial panel P), the Pll can be identified by being less hypointense than the other pulvinar nuclei and by its rather round cross-section, even though 
it is poorly delineated from the Plm. The inferior border of the pulvinar group has to be identified in coronal and sagittal views.

Sagittal slices provide an overview on the vertical extension of the pulvinar (sagittal panels TW). Just lateral of the MD (sagittal panel T), the hypointense pulvinar stands out clearly from the more anterior ventral nuclei. The medio-posterior apex of the thalamus is taken up by the Plm (as described above) that merges into the Pla. The inferior and superior borders of the Pla are poorly defined, as it appears to continue into the suprageniculate nucleus ( $\mathrm{Sg}$, posterior complex see 4.5.3) and into the LP, respectively. The Pll is seen only in the more lateral sagittal slices (sagittal panels $\mathrm{V}, \mathrm{W}$ ) that also show the striae between caudate and putamen. The tenuous hypointensity ventral of the Pll is assigned to the Pli and the LP is located dorsally of the Pll in sagittal panel V.

The Pli can be found in the horizontal slice that runs through the brachium of superior colliculus and slightly inferior of the intercommissural level (0-3.5 mm depending on the subject; axial panel Q). This landmark slice contains the most inferior parts of the thalamus as well as most superior structures of the midbrain. Here, the hypointense Pli can be located next to the cisterna laminae tecti and opposite the hippocampus. The shape of the Pli shows some individual variation. In addition, the Pli seems to merge into the lateral geniculate body (LG, see 4.5.3). It is therefore necessary to check the Pli in the corresponding orthogonal views to avoid confusion with the LG. Towards anterior, the medial geniculate body (MG) is consistently separated from the Pli by a hyperintense lamina. In coronal slices, the Pli merges dorsally and laterally into the Pll (coronal panel I) and thus appears as an integral part of the thalamus. Note that the Pli is cut at an angle in the axial slices and thus appears wider than in coronal slices.

\subsubsection{Geniculate NuClei ANd Posterior Complex}

The common terms of medial geniculate body (MG, Figure 25) and lateral geniculate body (LG, Figure 24) are used in this study. The Jones' classification differentiates between the dorsal and ventral nuclei of the medial geniculate complex (MGd and MGv), but these could not be distinguished at the chosen resolution. The pregeniculate nucleus (belonging to the ventral thalamus) cannot be resolved due to its small size and its merging with the LG in MT maps.

The hypointense LG can easiest be located in coronal slices at the position of the PC (coronal panel H). It is conspicuously more lateral than the mass of the lateral nuclei and separated by "being enveloped in the optic tract" (Jones 2007, p. 931). Its basis lies adjacent to the cisterna lamina tecti and as Jones describes fittingly has the "shape of an inverted U" (2007, p. 931). The 
laminar structure of the LG cannot be resolved and its periventricular lateral apex is disguised by partial volume.

In the corresponding horizontal slice (slightly inferior of the AC-PC level; axial panel Q) the LG merges medio-posterior with the Pli (see 4.5.2). The border between these two nuclei can occasionally be perceived as a narrow laminar structure, but is not resolved consistently (seen in 10/20 right, 3/20 left thalamus). The sagittal aspect of the LG is found next to the horizontal cisternal boundary. The sagittal panel W cuts the nucleus where it is separated from the Pll by a faint constriction.

Like the LG, the MG can be easiest seen in coronal slices at positions of the PC (20/20 subjects; coronal panel $\mathrm{H}$ ) between the LG and the brachium of superior colliculus. The hypointense $\mathrm{Sg}$ covers the MG like a cap, but there is no discernible border to the MG. Since these two nuclei show little contrast difference, they have to be assigned by their relative position. In the MT maps the $\mathrm{Sg}$ and the $\mathrm{CM}$ are seen in one coronal slice (coronal panel $\mathrm{H}$ ) even though in histological preparations the CM disappears when the Sg appears (Schulz-Schaeffer et al. 2008, private communication). It seems that this particular coronal panel $\mathrm{H}$ cuts the margin of CM.

The inferior portion of the MG can be clearly seen in the same horizontal slice used for locating the Pli (axial panel Q). This landmark slice contains the three distinct oval structures, which are embedded in hyperintense surroundings (seen in all subjects). The most posterior of these comprises the Pli and LG (see above). The most anterior is assigned to the ventral nucleus complex (see 4.7). The structure in between comprises the MG (laterally) and the Sg (medially). The anterior part of the white matter surrounding the MG-Sg hypointensity belongs to the lemniscus medialis; the posterior part to the brachium of superior colliculus. These WM tracts enclosing the MG can also be retrieved from the corresponding sagittal slice (sagittal panel U). Here, the MG is located next to the cisterna laminae tecti as a part of a hypointense round structure, the superior part of which belongs to the Sg. The hyperintensity anterior of the MG is the lemniscus medialis and the inferior part the brachium of inferior colliculus.

The $\mathrm{L}$ and $\mathrm{Sg}$ are the only nuclei of the posterior complex that can be identified. According to its name, the $\mathrm{L}$ is found at the border to the brachium of superior colliculus (coronal panel I; axial panel L). 


\subsection{NUCLEI ASSOCIATED WITH THE IML}

Within the IML, aggregations of neurons can be detected by histochemical staining (Hirai and Jones 1989; Morel et al. 1997). These nuclei are of different size and are dispersed along the whole course of the IML. The contrast of the IML is reduced in its posterior branch by unresolved intralaminar nuclei - the central lateral nucleus and the paracentral nucleus. The nuclei that can be detected in the MT maps appeared either hypointense or hyperintense. They will be identified from anterior to posterior following the extension of the IML as this is consistent with the classification into anterior and posterior intralaminar nuclei.

\subsubsection{ANTERIOR GROUP OF INTRALAMINAR NUCLEI}

The central medial nucleus (CeM, Figure 28 ventral aspect; Figure 29 anterior aspect) is found medial to the MTT. It encompasses the anterior apex of the MD. Therefore, it appears in several slices in different positions. An anterior and a ventral aspect can be identified in most subjects (sagittal panel R; 17/20 anterior, 20/20 ventral). The anterior CeM has been discussed in the context of the MV (see 4.3).

The most ventral aspect of the CeM can be found in coronal views at a position just posterior of the MTT (coronal panel D). Here, the ventral CeM extends from the third ventricle to the ventral nuclei like a narrow hypointense wing running along the ventral border of the MD. At the commissural level and just above - in some subjects as well below (axial panels $\mathrm{O}, \mathrm{P}$ ) it is seen as a hypointense area posterior of the MTT. Direct assignment of the anterior and ventral portion of the CeM in the sagittal panel $\mathrm{R}$ and $\mathrm{S}$ was not possible, but it can be localized indirectly by the orthogonal views. The ventral CeM appears more hypointense in the MT maps than other thalamic structures.

The magnocellular ventral anterior nucleus (VAmc, Figure 30) could not be consistently resolved. It is described embracing the MTT at the level just below the interthalamic adhesion (coronal panel C; axial panel N; sagittal panel S; Hirai and Jones 1989). In these views a slight hypointensity is seen that enhances the contrast to the MTT. 


\subsubsection{Posterior Group of InTRalaminar NuClei}

The centre médian nucleus (CM, Figure 31) is relatively large. It stands out in each orientation as a diffuse hyperintense area in the centre of the thalamic mass. In the axial views, it is at the posterior end of the IML and surrounded by the MD, the Pf, the Pla and the ventral nuclei (axial panels $\mathrm{N}, \mathrm{O}$ ). In the coronal slice it is best seen at the position of the habenular nucleus (coronal panels E-H), whereas it stands out less clearly in sagittal view (sagittal panel T).

The parafascicular nucleus (Pf, Figure 32 ) is easily assigned to the hypointensity posterior to the ventral CeM. This is located at the commissural level in transversal and sagittal views (axial panels $\mathrm{O}, \mathrm{P}$; sagittal panels $\mathrm{R}, \mathrm{S}$ ). At the border to the third ventricle, the Pf often appears to merge into the CeM that is directly anterior to the Pf (13/20 subjects). In the coronal view, it is seen at the inferior border of the thalamus between the red nucleus and the MD (coronal panel F). Although the contrast of the Pf is similar to the MD and no clear border is seen, the oblique protrusion of the Pf from the ventricle to the hyperintense mass of the $\mathrm{CM}$ reinforces the impression of two different structures.

\subsection{VENTRAL NUCLEI}

Despite their name, the ventral nuclei are located lateral of the IML and extend to the dorsal surface of the thalamus. Although part of the lateral group (4.5), they are covered in a separate chapter because most of their descriptions refer to the already mentioned nuclei. The ventral group consists of large nuclei, which appear generally less hypointense than the nuclei of the medial and pulvinar groups. The ventral nuclei exibit relatively poor intrinsic contrast (especially in sagittal views) and only few have laminar boundaries. Therefore, the extent of a nucleus can often not be exactly determined by structural MR. However, the relative position of all ventral nuclei can be assigned using other thalamic structures as landmarks and the orthogonal viewing. The most anterior nucleus of the lateral group is the ventral anterior nucleus (VA, Figure 33). The VA can be located in either orientation (coronal panels B-D; axial panels K-O; sagittal panels S-U). A hypointense portion of the VA can be found in coronal views lateral of the AV (coronal panels B-D). In these slices, the VA takes up the superior portion of the lateral mass next to the lateral branch of the IML. This nucleus shows a clear contrast gradient with the medio-ventral portion being more hypointense and the latero-dorsal portion less hypointense (coronal panels B, C; seen in 15/20 subjects). This inhomogenous impression is probably due to the density of axons running into the IC as Hirai and Jones mention (1989, p. 6f.): "The anterior pole of the region lateral to the internal medullary lamina $[\ldots]$ is perforated by numerous bundles 
of unstained axons." This region, therefore, does not represent different nuclear territories. The VA seems to merge ventrally with the hyopothalamus, the CeM (coronal panel B; sagittal panel S) and with the VM (coronal panel C; sagittal panel T). At positions where the VA merges with the ventral lateral anterior and posterior nucleus (VLa, VLp; coronal panel D; sagittal panel $\mathrm{U})$, the transition of the AV into the LD provides some orientation as AV and VA always appear together in the same coronal slices (Schulz-Schaeffer et al. 2008, private communication). In the horizontal view, AV is located adjacent to the lateral branch of the IML and forms the anterior pole of the thalamus (axial panels K-M). Anterior to the MTT in horizontal slices, the extent of the VA can be followed down to the hypothalamus slightly above the AC-PC level (axial panel O). The sagittal view shows a featureless hypointensity anterior to the MTT (sagittal panel S). This vertical expanse includes the VA without presenting clear cut borders towards superior (AV) and inferior (CeM and hypothalamus). In more lateral views (sagittal panels $\mathrm{T}, \mathrm{U}$ ) the VA blurs into the adjacent structures of VLa/p.

The principal ventral medial nucleus (VM, Figure 34) extends from lateral of the MTT to posterior. The anterior portion of the VM is found in the coronal slice showing the MTT (coronal panel C). It gets narrower towards its inferior apex, which is formed by the MTT and an oblique hyperintense band forming the inferior border of the thalamus towards the STN (Figure 6). The VM lies thus superior of the STN. As shown in Figure 12 coronal panel C, a slightly hyperintense oblique band can often be made out giving the impression of a border between VA and VM (seen in right thalamus 15/20, left thalamus 16/20). However, this band should be assigned to the VM, because from histology is known that the VM merges into the VA and the ventral lateral anterior nucleus (VLa) at more superior levels.

In axial and sagittal views the VM is not directly evident due to lack of contrast and has to be located using the crosshair. In transversal views, the VM can be traced at the AC-PC level by starting at the markedly hypointense hypothalamus running obliquely along the dot of the MTT (axial panel P). Adjacent to the MTT, the VM appears more hypointense where it merges with the much smaller VAmc (see 4.6.1). The posterior end of the VM is approximately located at the position of the interthalamic adhesion (seen in 18/20, 2 subjects without interthalamic adhesion). In sagittal slices somewhat lateral of the MTT, the VM can be found below the more hypointense VA (sagittal panel T).

The ventral lateral anterior nucleus (VLa, Figure 35) and the ventral lateral posterior nucleus (VLp, Figure 36) are located between MD and IC approximately within the middle 1/3 of the anterior to posterior extension of the thalamus. The two nuclei cannot be discerned and only roughly localized by prior knowledge from histology: The VLa is found more anterior and lateral, the VLp more posterior and medial. When progressing from anterior to posterior, the 
lateral extent of VLa gets narrower as the VLp gets wider. In the transition zone the nuclei are labeled as $\mathrm{VLa} / \mathrm{p}$.

The inferior part of the VLa can be found in the coronal view just posterior of the MTT (coronal panel D). It shows as a diffuse hypointensity next to the IC with strongly reduced contrast towards inferior. This change in contrast does not indicate different nuclear territories. The reduced hypointensity indicates a large number of passing axonal fibres, which corresponds to the description of the VLa in Jones (2007, p. 813): “ [...] VLa is characterized by being traversed by many thick myelinated bundles [...]." The corresponding axial slice (axial panels $\mathrm{N}, \mathrm{O}$ ) also shows an intensity gradient within the VLa/p that reduces the contrast to the IC. The lateral border to the IC can only be discerned at more superior levels (above level of the interthalamic adhesion), where to the VLa/p appears more hypointense (seen in 20/20 subjects; axial panel M). In coronal panel D, the VLa is not delineated against the neighbouring VA and VLp. At more posterior positions (coronal panel E), the inferior VLa is replaced by the VPL (see below).

The most difficult regions of assignment in the thalamus are the transitions between VLa and VLp, VLa and VPL, as well as VA and VLp.

Approximately $5 \mathrm{~mm}$ posterior of the intercommissural midpoint, the VLp takes up a major portion of the superior cross section of the lateral part of the thalamus. It extends laterally from the IML to the IC and from the dorsal surface of the thalamus towards the lateral inferior border (coronal panel E). Only the latero-inferior apex is filled out by the ventral nucleus complex (see below). In this and the neighbouring coronal slices, the inferior and lateral borders of the thalamus display a conspicuous angle of about 125 degree.

In the more inferior horizontal views (axial panel O) at the position of the Pf, a diffuse area of slight hypointensity is seen in the lateral thalamus. This area coincides with the coronal 125 degree apex assigned to the ventral nucleus complex.

The ventral posterior lateral nucleus (VPL, its anterior and posterior parts are combined in this study) and the ventral posterior medial nucleus (VPM) constitute the rather large ventral nucleus complex (Figure 37). The complex is best located in the horizontal and sagittal views in the slightly hyperintense area directly anterior of the hypointense pulvinar (axial panels N-Q; sagittal panels U, V; coronal G, H). The VPL and the VPM can only be discerned from each other by their relative position, which corresponds to their anatomical labelling: the VPL is located adjacent to the IC, the VPM adjacent to the CM.

The VPL shows a strong intensity gradient from ventral to dorsal. Its inferior apex is located slightly beneath the commissural level (axial panel Q) where it forms an oblique, strongly hypointense structure anterior to the MG. It often seems to extend laterally into the IC (axial panel Q), where the hypointense spot reflects the pyramidal tract. The inferior VPL is easy to 
identify also in sagittal view (sagittal panel $U$ ) where its hypointense mass is found anterior to the MG and Sg separated by myelinated structures. Thus, one can trace its location even in coronal views, where it is less conspicuous. Here, the hypointense inferior part of the VPL may be mistaken as a separate structure or a lateral extension of the substantia nigra (coronal panel $\mathrm{G)}$.

Towards dorsal the hypointensity of the VPL/VPM decreases strongly. Thus, further delineation of the ventral nucleus complex becomes increasingly ambiguous until it abuts onto the more hypointense VLp and LP (coronal panel G; sagittal panel U).

The ventral posterior inferior nucleus (VPI, Figure 38) can best be localized in horizontal views at the AC-PC level (axial panel P). It appears adjacent to the IC, intercalated between the basal ventral medial nucleus (VMb, Figure 39) and the VPL. In the coronal view, the VPI is located at the lateral-inferior border, which appear slightly diffuse at this position (coronal panel F). The VPI is covered dorsally by the VPL. It borders medially on the CM and on the VMb below the CM. The lateral border of the VPI is hardly to distinguish from the pyramidal tract in the IC. In sagittal views, the VPI can be located indirectly by the inferior part of the VPL serving as a landmark. Here the hyperintense VPI is situated superior and slightly anterior of the hypointense VPL (sagittal panel U). Once again the hyperintense area in MT maps indicates the high amount of axonal fibres running through the VPI. It is transversed by the medial lemniscus as it enters the thalamus (Jones 2007).

The basal ventral medial nucleus (VMb) can be found at the commissural level lateral of the Pf and between the CM and VPI (axial panel P). It runs obliquely between VPL and the Pf. In coronal view it is seen as hypointensity between the CM and the inferior border of the thalamus and located between the more hypointense Pf and the less hypointense VPI (coronal panel F). In sagittal views (sagittal panel T) the VMb must not be confounded with the inferior apex of the VPL which is found more lateral (sagittal panel U). Both nuclei are at the inferior apex of the broad hypointensity.

Of special note: a pattern of broad stripes of alternating contrast can be seen in sagittal views (sagittal panel T, U). Here, the indistinct hypointense wedge is mostly assigned to the VLp. It is pointing down towards the VMb (sagittal panel T) or VPL (sagittal panel U). Towards anterior the VLp raises above parts of the VA and towards posterior it bends over the LP and the ventral nucleus complex. 


\subsection{LANDMARK SLICES}

In retrospect, a number of slices can be identified by anatomical landmarks, which contain a wealth of information about the thalamic substructures and are thus pivotal for assignment. These landmark slices are the:

I) coronal view through the MTT (panel C);

II) coronal view with the 125 degree angle of the latero-inferior thalamus border (panel E);

III) coronal view through MG and LG (panel H);

IV) axial view with the level of the interthalamic adhesion (panel M);

V) axial view with the intercommissural level (panel P);

VI) sagittal view with the extent of the MTT (panel S);

VII) sagittal view through the MG (panel U).

With the help of these landmark slices, the majority of thalamic nuclei can be identified. 


\section{DISCUSSION}

„So ist denn auch der Hirnbau in seinen feinsten Einzelnheiten ein an sich wichtiger Gegenstand naturwissenschaftlicher Untersuchung, unabhängig von aller Anwendbarkeit, die darum nicht ausbleibt, sollte sie auch nicht sogleich hervortreten. Und mit solcher Ueberzeugung, dafs wir es hier nicht mit einer unfruchtbaren Grübeley zu thun haben, mögen wir an das Studium der Hirnlehre gehen."

Burdach, (1822, p. 1)

\subsection{INTERCOMMISSURAL REFERENCE SYSTEM AND SYNOPTIC ORTHOGONAL VIEWING}

In this thesis, the intercommissural line was chosen as horizontal reference for the $3 \mathrm{D}$ data because it is commonly used in stereotaxis, and in the recent digital atlases (Niemann et al. 2000; Krauth et al. 2010). The coronal slices that were used for assignment (Schaltenbrand and Wahren 1977; Hirai and Jones 1989; Morel et al. 1997; Jones 2007; Mai et al. 2008) abide closely to the AC-PC orientation. Complications arise in that the horizontal preparations of these atlases are inconsistently angulated. The lower AC to upper PC alignment first defined by Talairach (1957) and adopted in the Schaltenbrand and Wahren atlas (1977) deviates by about 7 degree (Niemann et al. 1994) to the intercommissural plane that goes through the centres of AC and PC. This angle would correspond to a horizontal deviation of $3.5 \mathrm{~mm}$ (or seven $0.5 \mathrm{~mm}$ slices) along the anterior-posterior extension of the thalamus. The angulations of the horizontal planes in Hirai and Jones (1989) are roughly orthogonal to the brain stem axis. In principle, digital processing would allow to skew the 3D data volume by an affine transformation in order to accommodate the frontal planes with slanted non-orthogonal horizontal planes.

Synoptic orthogonal viewing has two major advantages when identifying single thalamic nuclei: A nucleus can be identified in the plane where it is most conspicuous and then landmarks can be defined in planes where the nucleus cannot be directly identified. The ventral nuclei, e.g., were identified mainly by landmarks or by their relative position. In the same manner, such landmarks can be used to check if a nucleus appears in another plane other than the one indicated in the 
histological atlases (see the MG in Figure 12 - Figure 14 and Figure 25) or to distinguish the transition between two nuclei since as the AV to LD is only clear in one orientation (see 4.4; Figure 17; Figure 18). Furthermore, often all three orientations are necessary to see the extent of a nucleus which further facilitates the identification. For example, when only viewing the horizontal panel Q the identification of the three conspicuous hypointense areas is difficult.

Of the three orientations, the coronal views provided the most valuable information, not at least because the position along the intercommissural line can be roughly inferred from the crosssection of the thalamus and surrounding landmarks. The MRI data is of much poorer resolution, where the slices are seen to run exactly parallel or orthogonal compared to histological preparation. This is owed to the high bandwidth of the multi-echo acquisition and because the subject are positioned with their heads at the isocentre of the magnet.

\subsection{STRUCTURAL MRI}

The appearance of the thalamus in the MT maps can be transferred to other "structural" MRI data to the degree that depends on the resolution and the contrast-to-noise ratio. Structural MRI is usually based on T1-w contrast since the T1 relaxation times of tissue are dominated by the amount of macromolecules. Though the contrast between GM and WM is mainly due to the presence of myelinated axons the iron content of tissue shortens T1 (Gelman et al. 2001). This reduces the contrast of iron containing deep brain nuclei to adjacent WM, notably the STN and the globus pallidus (Helms et al. 2009b). The thalamic nuclei may differ in iron content, which is especially high in the Pll, as seen by a high R2* or in Abosch et al. (2010). Magnetization transfer contrast is solely based on the interaction of water with macromolecules. The semiquantitative maps of MT saturation are proportional to the macromolecular fraction (Helms et al. 2008c). They are corrected for the underlying $\mathrm{T} 1$ and effective $\mathrm{T} 2$ relaxation and are, thus, independent of local iron content and water mobility. The measurement protocol strives to overcome the poor contrast-to-noise ratio within grey matter structures at the limited resolution achievable on clinical MR systems within acceptable measuring time (Gringel et al. 2009). In contrast to standard MRI images, the MT maps are reproducible within errors, since they are corrected for spatial variation of the receive coils and the flip angle. This allowed viewing the MT maps at a consistent grey scale. In principle, a pseudo-colour display could be used to enhance the small contrast differences. Nevertheless, the grey scale images presented in this thesis maintained the "natural" T1-like appearance to facilitate the comparison with other structural MRI techniques. Only the standard hardware of a clinical $3 \mathrm{~T}$ MR system and the 
manufacturer's spoiled multi gradient echo sequence were used, the novel reconstruction was scripted offline using freeware tools.

To boost the intra-thalamic contrast via the T1 relaxation, optimized inversion recovery MRI was initially proposed (Magnotta et al. 2000) and recently reinvestigated (Bender et al. 2011). Specific nuclei were consequently segmented by quantitative T1 and T2 mapping (Deoni et al. 2007). All these structurally based approaches should correspond well to the atlases that show myelin staining (Schaltenbrand and Wahren 1977; Mai et al. 2008). By far most segmentation approaches are based on local diffusion properties (Wiegell et al. 2003; Ziyan et al. 2006; Unrath et al. 2008; Ziyan and Westin 2008; Mang et al. 2011). The diffusion tensor imaging (DTI) describes the main direction and coherence of axons, but usually at lower resolution than structural MRI. The MT saturation may serve as a high resolution complement to enhance the specificity of DTI in the thalamus.

The MT maps are highly sensitive to lesions in the thalamus, as can be seen in multiple sclerosis or after stroke (not shown).

\subsection{MT Maps, Classical and Population Atlases}

The traditional atlases (e.g. Schaltenbrand and Wahren 1977; e.g. Mai et al. 2008) are based on histological preparations and the orthogonally series come from different brains. Therefore, the atlases have their limits when it comes to assigning individual anatomy in vivo.

Aspects that have to be considered when comparing or even digitizing the microscopic slices are:

- horizontal, coronal and sagittal 2D slices do not correspond in 3D space (Niemann and van Nieuwenhofen 1999);

- deviating definitions of the AC-PC reference plane (Talairach 1957; Percheron et al. 1986). Differences between the atlases were found mainly with regard to the transversal planes (see 5.1);

- $\quad$ singular nuclei may be inconsistently labeled (Niemann et al. 1994; Bogen 2006);

- technical inaccuracy during cutting of the preparations (Hirai and Jones 1989; Niemann et al. 1994);

- individual variance of "normal" morphology and size of brain (Raz et al. 2005) as well as asymmetries of cerebral hemispheres (van Buren and Borke 1972; Morel et al. 1997).

Until recently, histological atlases have been in book form, the classical medium for disseminating state-of-the-art neuroanatomical knowledge. The rapid progress in digital neuroimaging techniques has lead to a change in paradigm, featuring digital 3D atlases which 
may even represent variances in populations (Toga et al. 2006). This thesis uses 3D data from individuals.

The advantage of MR imaging is that it is non-destructive. It can be applied in vivo, post mortem and on a fixated specimen prior to cutting. The challenge for the live subject is to remain still during scanning in order to avoid motion artefacts. As a result, a short examination time is important.

Morel et al. (1997) use the same terminology as Hirai and Jones (1989), but eventually simplified the nuclear groups (Krauth et al. 2010). 1) Only the ventral nuclei are assigned to the lateral group. 2) A posterior group comprises the pulvinar nuclei, the geniculate nuclei and the posterior complex. 3) The nuclei of the intralaminar group as proposed by Hirai and Jones are split up into the four groups (medial, posterior, lateral, anterior group). In this thesis, 25 of the 31 nuclei of Morel' classification were identified.

The overlay of MRI data with the camera lucida drawings from Jones 2007 illustrates the limitations in spatial congruence of 2D preparations with 3D MRI (Figure 6 and Figure 7). Rotation, translation, scaling and skew (6 parameters) were to be adjusted manually but, a match as good as Figure 6 and Figure 7 was not possible in each of the 20 subjects. This was mainly due to the fact that the reticular nucleus cannot be detected in the MT maps and therefore the lateral thalamic border of the overlay could not be exactly delineated. The horizontal camera lucida drawings are not shown because they did not match the AC-PC orientation but were tilted by about 17 degrees towards antero-ventral. At this angle, they were roughly orthogonal to the brain stem axis of Meynert, and not matching the forebrain axis of Forel. Arbitrary reference to these axes has been identified as a source of confusion in the midbrain (Coenen et al. 2008). Even the frontal slices showed some spatial mismatch: The two camera lucida drawings shown in Figure 6 and Figure 7 are those that conformed closest to slices orthogonal to the AC-PC line. It was also observed that parts of STN and RN did not appear in the same coronal slice in the AC-PC reference system, in contrast to the camera lucida drawing H6-47 (Jones 2007, p. 1431, Figure 18.6). Also in the atlas of Mai et al. (2008, p. 164, Figure 36), STN and RN are shown in one slice. In Figure 6 and Figure 7, R2* provided additional information over the MT maps, which was used to match the STN of the overlay.

Therefore, a high-resolution MRI scan of a fixated brain specimen prior to histological preparation is recommended to verify the assignment of the thalamic nuclei and to resolve arbitrary spatial relationships mentioned above. MRI data has been instrumental for the spatial registration of individual histological data compiled into a common digital atlas (Krauth et al. 2010). 


\subsection{CONTRAST AND RESOlution}

In this thesis a report on individual thalamic anatomy is conducted. Reference to contrast is made with respect to the most conspicuous features and does not necessarily apply to the whole extent of the nucleus. So far, MRI data does not provide information on the extent or borders of singular nuclei, which corresponds to everyday experience in clinical MRI. Therefore, no nucleus specific statistics of size and location can be given. This limitation is ameliorated only in part at $7 \mathrm{~T}$ and very high resolution. For instance, susceptibility weighting shows a distinct medial to lateral contrast gradient through the pulvinar nuclei (Abosch et al. 2010).

The image resolution of $0.95 \mathrm{~mm}$ provided the basic scale of resolution. Compact nuclei of low axonal density (like the MG) appeared hypointense compared to the surrounding laminae. Minor nuclei were disguised by partial volume effects, especially when neighbouring to tracts of white matter as "seen" in the intralaminar group. Similarly, the minor bundles of axonal fibres in the lateral thalamus (and the putamen) could not be resolved. Such regions show a finely graded intensity but likely represent both smaller tracts and dispersed axonal connections. Major axonal tracts and laminae, like the IML, could be discerned if their dimension was the order of resolution. Still, their MT values were lower than those of compact WM. This likely reflects variable partial volume effects with adjacent nuclei/GM structures (for example anteromedial and anterodorsal nucleus, parataenial nucleus). In general, such tenuous WM structures showed a rather large variation in intensity. The magnocellular nuclei appeard slightly hypointense, but since MT contrast is dominated by the axonal content, no clear correlation with neuronal size could be established (e.g. MD). Several of the ventral nuclei (especially VA, VLa, VPI see 4.7) appeared rather hyperintense which is due to the high content of the passing axonal bundles.

High vascularity (like in the HT) and major vessels, especially those with widened perivascular spaces, reduce the MT saturation as shown in Figure 7 of the work in Gringel (2009). Misassignment to a hypointense nucleus can usually be ruled out by geometrical argument. The detection of minor periventricular structures was rendered impossible by varying contributions of CSF.

Not only did the protocol of magnetization transfer (MT) ameliorate the contrast within the thalamus but also the contrast of the surrounding structures was improved. Parts of the midbrain are shown in panels (e.g. coronal panels C-I; axial panel Q; sagittal panels R-U), a detailed description of which is beyond the scope of this study. 


\subsection{Potential Benefits for Stereotaxic Applications}

Deep Brain Stimulation (DBS) has become an established symptomatic treatment of refractory diseases in neurology and psychiatry. Depending on the symptoms, DBS targets specific nuclei of the basal ganglia and thalamus: epilepsy (Ncl. anterior; Fisher et al. 2010), Parkinson disease (STN, globus pallidus internus, ventralis intermedius nucleus, CM-Pf, pedunculopontine nucleus; Krause et al. 2001; Benabid et al. 2002; Zrinzo et al. 2008), essential tremor (ventralis intermedius nucleus; Benabid et al. 1996), dystonia (globus pallidus internus; Pinsker et al. 2009), Gilles de la Tourette syndrome (ventralis oralis internus complex, CM-Pf, globus pallidus internus; Vandewalle et al. 1999; Welter et al. 2008), and peripheral deafferentation pain (nucleus ventralis caudalis; Yamamoto et al. 2006). The stereotaxic placement of DBS electrodes is likely to benefit from a better delineation of individual anatomy.

Functional segmentation of the thalamus plays an increasingly important role in basic neuroscience research. It has become clear that the cooperation between different fields of expertise: image analyses, MRI techniques such as DTI and fMRI, neuroanatomy, neurobiology, and neurosurgery, provide important contributions for a reliable radiological visualization of the thalamus.

In planning a stereotaxic surgery, particularly DBS, a standardized digital atlas is registered to the patient's MRI. Thus, the target is localized and coordinates are determined in a stereotactic frame of reference (Otsuki et al. 1994). However, as mentioned above, a degree of inaccuracy remains due to individual anatomic variability. This has to be corrected during surgery through clinical observation of the semi-awake patient and electrophysiological feed-back along the trajectory (Niemann and van Nieuwenhofen 1999; Abosch et al. 2010).

The distortion in the MT maps due to the gradient field of the MRI scanner, have to be corrected by calibration measurements or by non-linear morphing to a CT scan. The benefits for the patient then would be an optimized treatment with less adverse effects e.g. reduction in surgery time, reduction of complications such as symptomatic intracranial hemorrhage (Ben-Haim et al. 2009; Abosch et al. 2010), less misplaced electrodes and less unnecessary white matter damage.

Hassler established the V.im (Nuclei ventrointermedii) as target to ameliorate certain symptoms of Parkinson's disease (Hassler and Riechert 1954). Today, it is a target for DBS but there is no direct correspondence to the classification of Hirai and Jones. They stated in 1989 that the "ventral [...] part of the VLp is equivalent to the V.im of Hassler" (p. 21). However in 2007 Jones corrected his statement saying that V.im "be more correctly identified as VPLa instead of the posterior part of VLp." (p. 1439). In the MT maps, the ventral part of the VLp could not be precisely identified. However, its anterior neighbour, the VPL, could be identified in the MT- 
maps by two different features: 1) the latero-inferior angle in coronal views (coronal panel E), corresponding to the VPL in sagittal panel U intercalated between VPI and VLa/p and 2) the axial and sagittal views as a hypointensity anterior of the MG (axial panel Q; sagittal panel U). The horizontal plate 53 of the Schaltenbrand and Wahren atlas shows that the V.im. is located anterior to the V.c.e and V.c.i (Nucleus ventrocaudalis externus and internus). Thus, the V.c. would correspond to the VPL in axial panel $\mathrm{Q}$ and in sagittal panel $\mathrm{U}$ anterior of the MG; the V.im to the part of VPL that is intercalated between VPI and VLa/p in the sagittal panel U. Could the targeted V.im. correspond to the VPL delineated in the MT maps (Figure 9)? The identification of the V.im by reference to the literature remains hypothetical and the issue will need practical validation by the use of MT maps in clinical DBS applications. Furthermore, Jones raised the question whether "perhaps no single thalamic nucleus can be regarded as the effective tremor elimination target?" (2007, p. 1439).

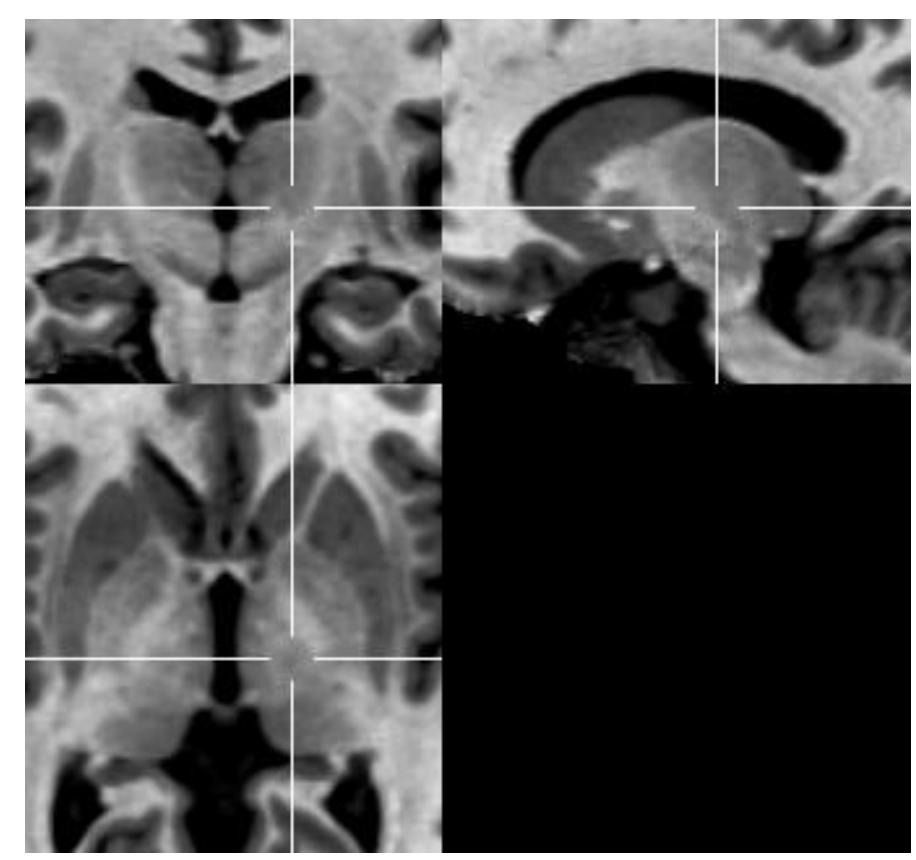

Figure 9: Hypothetical position of the V.im

Coordinates hum_1950 103-75-68: $18.5 \mathrm{~mm}$ posterior of AC, $12.5 \mathrm{~mm}$ lateral of origin, $1.5 \mathrm{~mm}$ dorsal of AC-PC level.

Aiming at a robust localization of the V.im or the STN, the MT maps did not provide a clear benefit over conventional T1-w MRI. This is not entirely surprising in view of the closely related contrast mechanisms. Contrary to the V.im, the STN and the RN can be located by their rapid effective $\mathrm{T} 2$ relaxation. These structures can be used as landmarks to locate the ventral thalamic nuclei in all three orientations. For instance, the ventral part of the VLp can be located at the same position as the posterior RN and posterior and slightly above of the lateral pole of the STN. 


\subsection{ChALLENGES AND Limitations}

The descriptions of the different nuclei in histological preparations fittingly describe the anatomy of the thalamus: Viewing the MT images could be accompanied by a neuroanatomy text book, e.g., by "The Thalamus" of Jones (2007). Moreover, the MT maps resemble certain histological preparations, especially those staining for myelin as described above and illustrated in Figure 10. As much as the MT maps and myelin stained histological preparations resemble each other, the differences are evident as well. Most obvious is the difference in resolution because motion artefacts from the subjects/patients reduce the sharpness of the MRI image and the limited number of MR contrast mechanisms (macromolecules, iron, axonal coherence) does not match the variety and specificity of immunohistochemical staining methods.

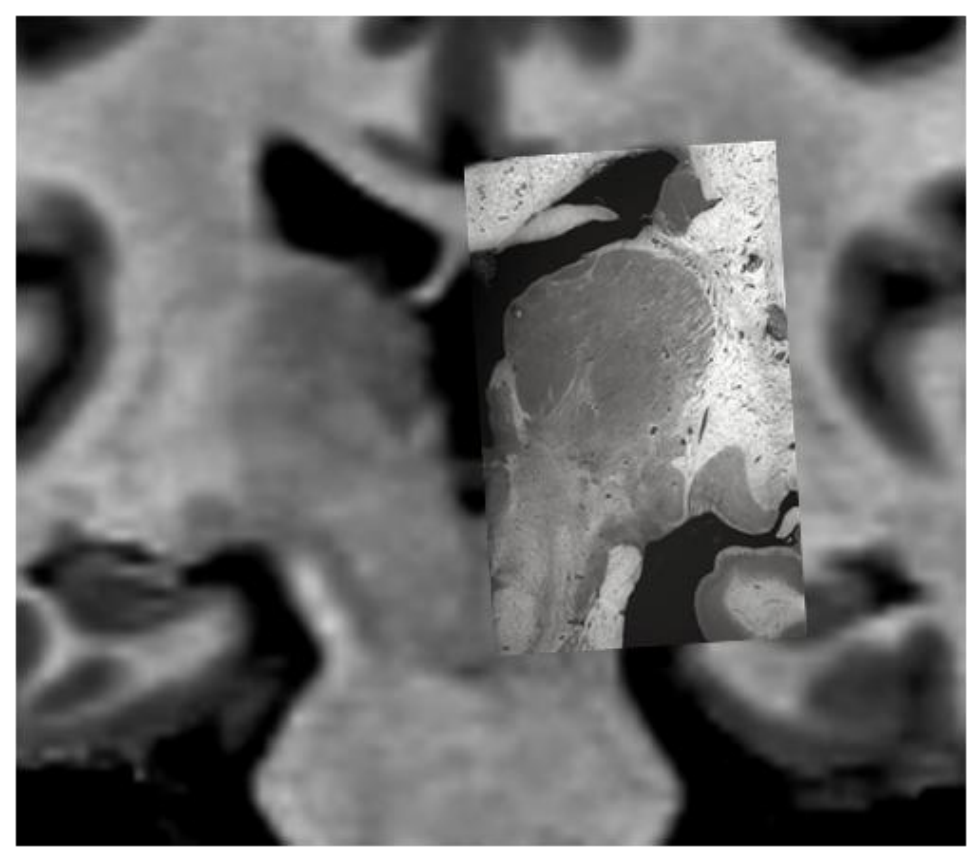

Figure 10: MT map resembling a histological section

Backroud: coronal view of MT map at $26.5 \mathrm{~mm}$ from AC (hum_1950, Y 59)

In the front: coronal histological section at $27.8 \mathrm{~mm}$ from AC (Mai et al. 2008, p. 178, Figure 43 inverted) For the delineation of nuclei see coronal panel $\mathrm{H}$ and p. 179 of the atlas of Mai.

Note: views resemble each other, however coronal view of MT map anterior of the pulvinar nuclei. 
Numerous variations exist concerning the nomenclature of the dorsal thalamus - especially for the ventral group of nuclei. This is challenging for the novice and a source of dispute between advanced neuroscientists: "More disagreement has probably been generated over the parcellation of the ventral nuclei than any other nuclei in the primate thalamus" (Jones 2007, p. 1423). This thesis does not discuss the benefits of different systems, but uses the North American terminology exclusively.

Because symmetry criteria play a major role in distinguishing small structures from artefacts asymmetries between the hemispheres may pose a problem. This rather common problem depends on the individual's anatomy and cannot be overcome by linear registration techniques. Non-linear deformation models may also face problems due to the marginal contrast differences observed in the thalamus. This may pose a general problem to the automatic delineation of nuclei based on MRI, since most of the thalamic nuclei were found lacking a homogeneous intensity and/or clear-cut borders. Future research should be directed to whether non-affine registration and construction of population averaged data benefits from the use of quantitative parameter maps.

The data in this thesis does not provide a population based atlas due to lack of available free ware tools. Inspired by four subjects presented by Deoni et al. (2007), one of the goals of this thesis was a multi-parametric characterization of the nuclei. Contrary to their work, the MRI data of this thesis did not establish clear borders or characteristic MT values and relaxation times of homogeneous nuclear territories (see 5.4). The nuclear boundaries of the work in Deoni et al. (2007) likely represent prior knowledge derived from the histological atlas of Niemann et al. (2000) and less specific MRI features. Only with the availability of population-based digital atlas nuclear boundaries (Krauth et al. 2010) and tools for non-linear registration ("fnirt" of later FSL versions), it may be possible to address the signal characteristics of singular nuclei. 


\section{SUMMARY}

"Most of the existing databases are in atlas format, which tends to impose a fixed view of brain organization and structure. This has its merits but it also has its limitations. Looking at the brain through the eyes of an atlas maker is like reading Aeschylus or Sophocles in translation. You can certainly gain an appreciation of the beauty of the language but if you want to appreciate its full grandeur, you had better go and learn it for yourself."

Jones (2007, p. 1465)

A technique for high-resolution multi-parametric MRI was optimized for application to the human thalamus in vivo at $3 \mathrm{~T}$ with a scanning time of approximately 25 minutes. The MT maps showed the highest contrast and were thus used to identify structures in 20 healthy adults.

25 of 34 thalamic nuclei according to Hirai and Jones' nomenclature could be detected at 3T and $0.95 \mathrm{~mm}$ isotropic resolution in the AC-PC reference system.

The results show that it is possible to image the individual anatomy of the thalamus by in vivo MRI and even its smaller substructures.

Some specific findings of general importance may be noted:

I) The IML serves as a landmark for the assignment of nuclear groups such as in stained histological sections.

II) The location of CM could be consistently identified.

III) The extent of the geniculate bodies is easily overestimated, since the MG tends to merge with the Sg and the LG with the Pli.

IV) Since the MT values correlates mainly with the myelin content, a correlation between cell size and MT could not be established beyond doubt.

V) At a resolution of $0.95 \mathrm{~mm}$ the lateral thalamus border is unclear due to high MT values in the ventral nuclei and the unresolved reticular nucleus.

This study may thus serve as a guide to the human thalamus and may it serve as a contribution to "gain an appreciation of the beauty" (Jones 2007, p. 1465) of the dorsal thalamus as the thalamus can no longer be regarded as inaccessible by MRI. 


\section{APPENDIX}

"I am reminded of the old story about the blind men and the elephant: each touched a different part of the elephant and had a different description of the whole animal. In a similar way, each of those who touch the thalamus will have a slightly different story to record."

Krieg (Dewulf 1971, p. 122)

\subsection{LIST OF ABbREVIATIONS}

$\begin{array}{llll}\text { AC } & \text { anterior commissure } & \text { GP } & \text { globus pallidus } \\ \text { AV } & \text { anteroventral nucleus } & \text { Gyu } & \text { gyrus uncinatus } \\ \text { CA } & \text { caudate nucleus } & \text { H } & \text { habenular nucleus } \\ \text { CC } & \text { corpus callosum } & \text { HF } & \text { high frequency } \\ \text { CeM } & \text { central medial nucleus } & \text { Hy } & \text { hypothalamus } \\ \text { Ci } & \text { brachium of inferior } & \text { IC } & \text { internal capsule } \\ & \text { colliculus } & \text { IML } & \text { internal medullary lamina } \\ \text { Cis } & \text { cisterna laminae tecti } & \text { L } & \text { limitans nucleus } \\ \text { CM } & \text { centre médian nucleus } & \text { LD } & \text { lateral dorsal nucleus } \\ \text { CP } & \text { cerebral peduncle } & \text { LG } & \text { lateral geniculate body } \\ \text { Cs } & \text { brachium of superior } & \text { LP } & \text { lateral posterior nucleus } \\ & \text { colliculus } & \text { LV } & \text { lateral ventricle } \\ \text { CSF } & \text { cerebrospinal fluid } & \text { MB } & \text { mamillary body } \\ \text { 3D } & \text { three-dimensional } & \text { MD } & \text { mediodorsal nucleus } \\ \text { DBS } & \text { deep brain stimulation } & \text { MG } & \text { medial geniculate body } \\ \text { DTI } & \text { diffusion tensor imaging } & \text { MRI } & \text { magnetic resonance imaging } \\ \text { FA } & \text { flip angle } & \text { MT } & \text { magnetization transfer } \\ \text { F } & \text { bound proton fraction } & \text { MTR } & \text { MT-Ratio } \\ \text { Fx } & \text { fornix } & \text { MTT } & \text { mamillo-thalamic tract } \\ \text { GM } & \text { grey matter } & \text { medioventral nucleus } \\ & & \end{array}$


$\mathrm{Mz} \quad$ longitudinal magnetization

OCX optic chiasm

OT optic tract

PC posterior commissure

Pcs superior cerebellar peduncle

PD proton density

Pf parafascicular nucleus

$\mathrm{Pg} \quad$ pregeniculate nucleus

$\mathrm{Pla} \quad$ anterior pulvinar nucleus

Pli inferior pulvinar nucleus

Pll lateral pulvinar nucleus

Plm medial pulvinar nucleus

PT pyramidal tract

$\mathrm{Pu} \quad$ putamen

$\mathrm{RN} \quad$ red nucleus

Sg suprageniculate nucleus

SN substantia nigra

SNR signal-to-noise ratio

STN subthalamic nucleus

$\mathrm{T}$ tesla

TE echo time
TR repetition time

$3 \mathrm{~V}$ third ventricle

V.im nucleus ventro-intermedius

VA ventral anterior nucleus

VAmc magnocellular ventral

anterior nucleus

VLa ventral lateral anterior

nucleus

VLp ventral lateral posterior

nucleus

VM principal ventral medial

nucleus

$\mathrm{VMb}$ basal ventral medial nucleus

VPI ventral posterior inferior

nucleus

VPL ventral posterior lateral

nucleus

VPM ventral posterior medial

nucleus

w weighted

WM white matter 


\subsection{The Dorsal Thalamus NuClei ON MT MaPS}

The MT maps of the thalamus shown in the Figures are all taken from the subject hum_1950; except Figure 6, 7 and 8.

The coordinates given in the orthogonal views are labelled as provided by MRIcro by the coordinates of the intersection of sagittal-coronal-horizontal which equivalents to X-Y-Z. These coordinates can be related to the origin of the stereotactic coordinate system, chosen at the midpoint of the intercommissural line (midpoint in hum_1950 in the coronal view Y85, $13.5 \mathrm{~mm}$ posterior from AC). The AC-PC distance was $27 \mathrm{~mm}$ in subject hum_1950 (the distances of the other subjects were between $25 \mathrm{~mm}$ and $29 \mathrm{~mm})$. The voxel size is $(0.5 \mathrm{~mm})^{3}$ and division by two yields the distance in $\mathrm{mm}$.

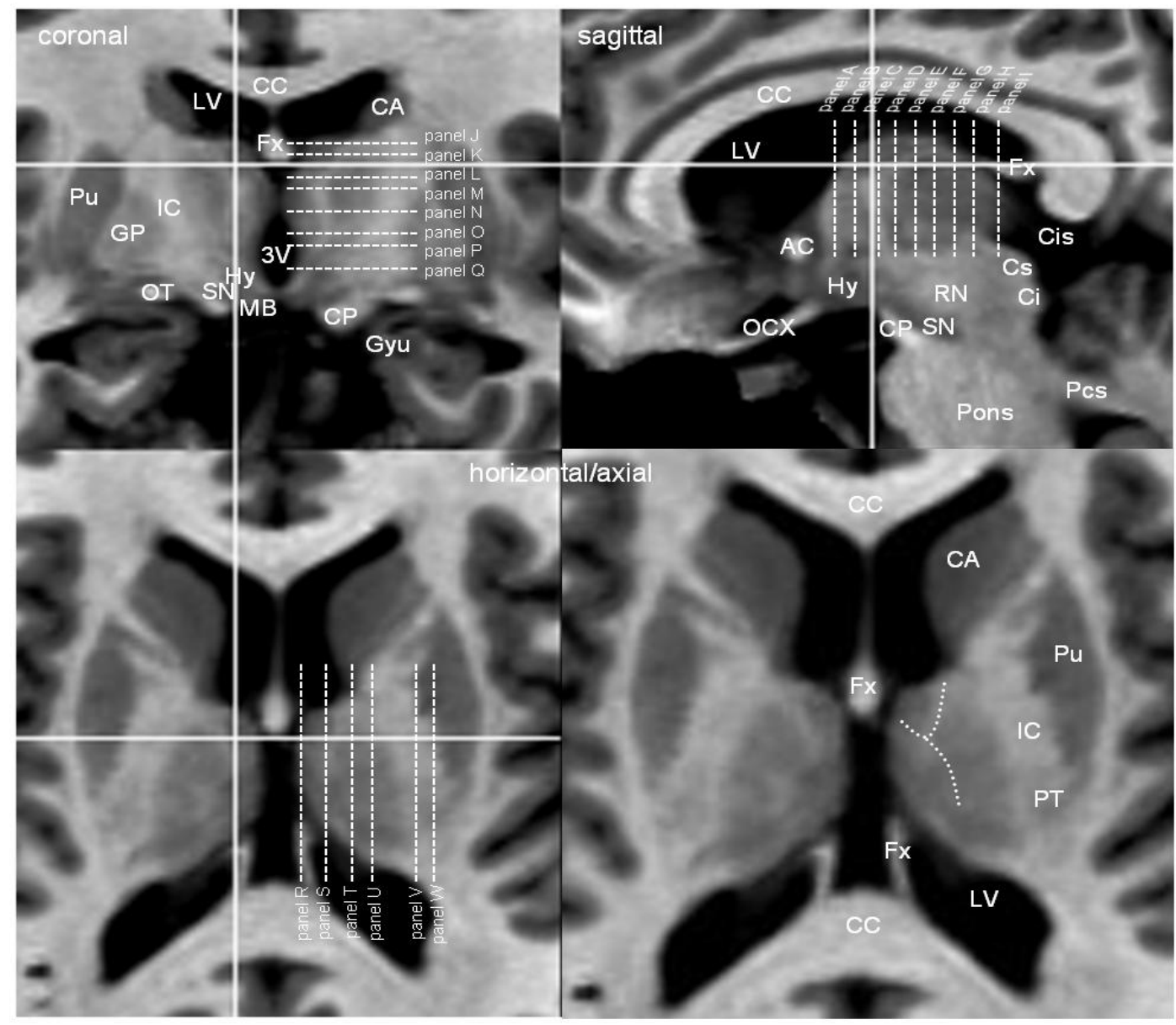

Figure 11: Orthogonal display of hum_1950 at coordinates 65-89-91

Localizer of the panels A-W and non thalamic structures.

The crosshair showing the equivalent positions in the planes.

Enlarged horizontal plane: Internal Medullary Lamina (IML, dotted line) 


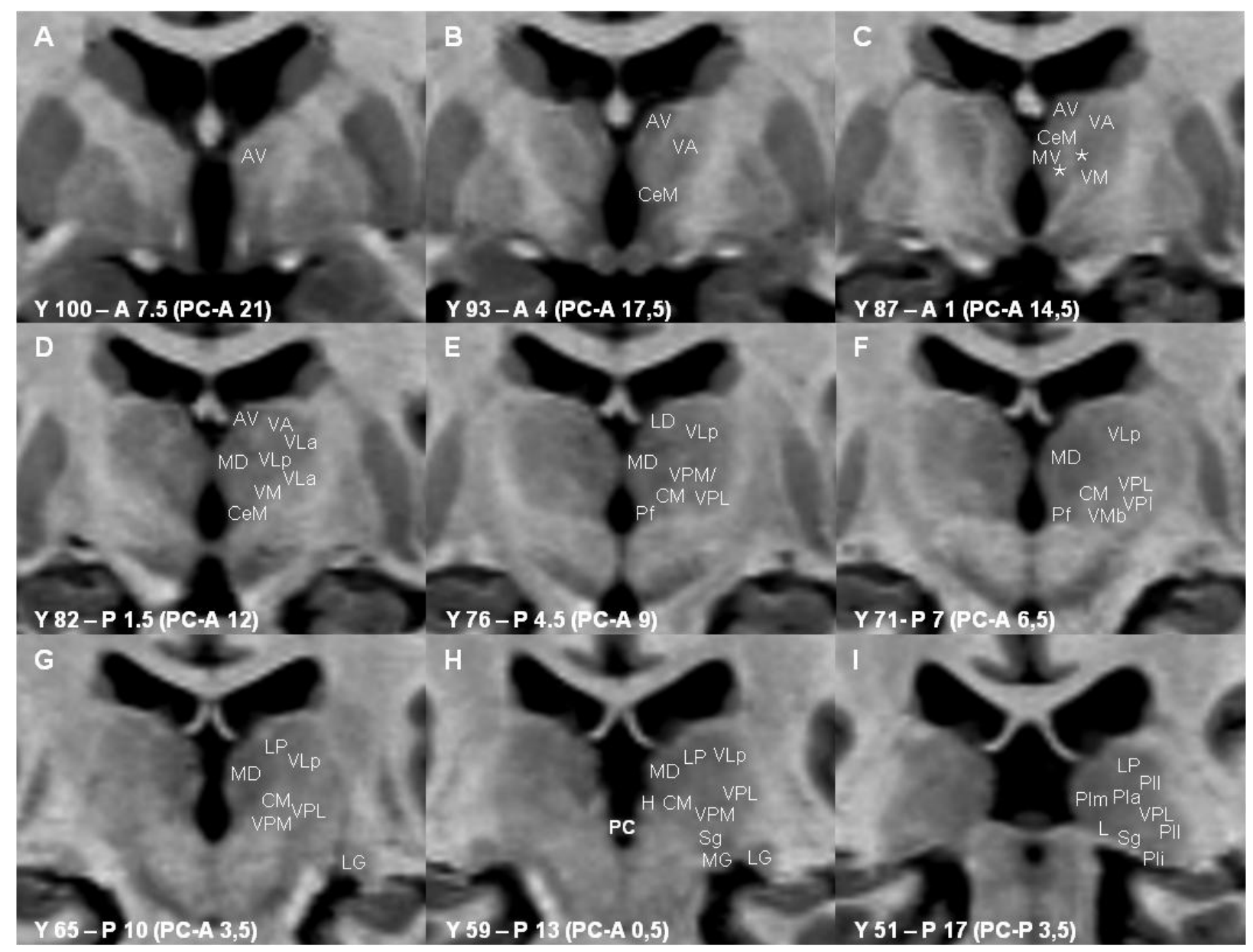

Figure 12: Coronal panels

Continuous slices of thalamus MT maps in anterior to posterior order.

Orientated perpendicular to AC-PC level.

Y-coordinates derived from MRIcro.

Coordinates indicated in millimetres anterior $(\mathrm{A})$ or posterior $(\mathrm{P})$ of origin at $\mathrm{Y} 85$.

In parenthesize coordinates $\mathrm{A}$ or $\mathrm{P}$ of the posterior commissure (PC) in $\mathrm{mm}$.

Compare left with unmarked right hemisphere.

Position of interthalamic adhesion in panel C, D, E. Position of PC in panel H.

*: VAmc. 


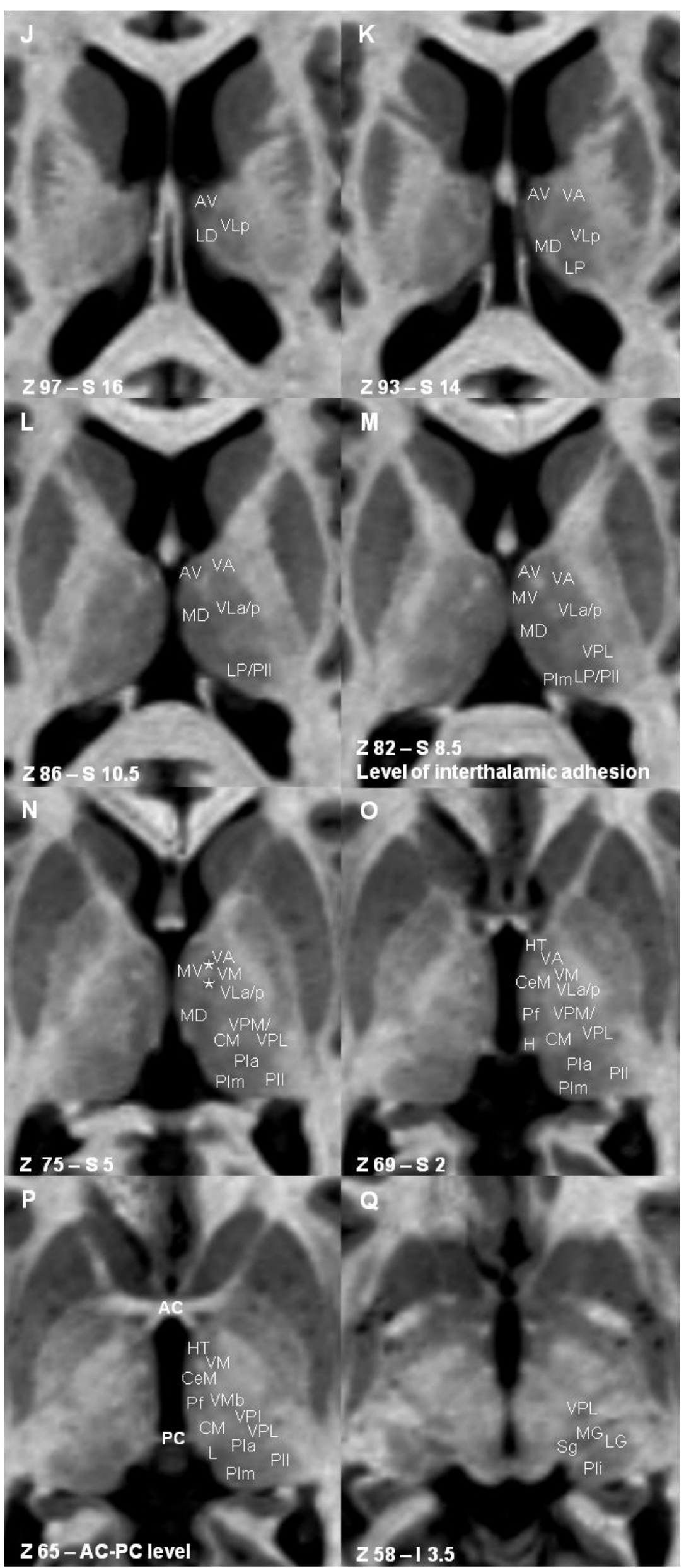

Figure 13: Horizontal panels

Continuous slices in superior to inferior order.

Orientated parallel to

intercommissural plane (AC-PC level).

Z-coordinates derived from

MRIcro.

Coordinates indicated in

millimetres superior $(\mathrm{S})$ or

inferior (I) of AC-PC level at Z

65.

*: VAmc.

Cave: horizontal views have not the same angulation as Hirai and Jones (1989). 


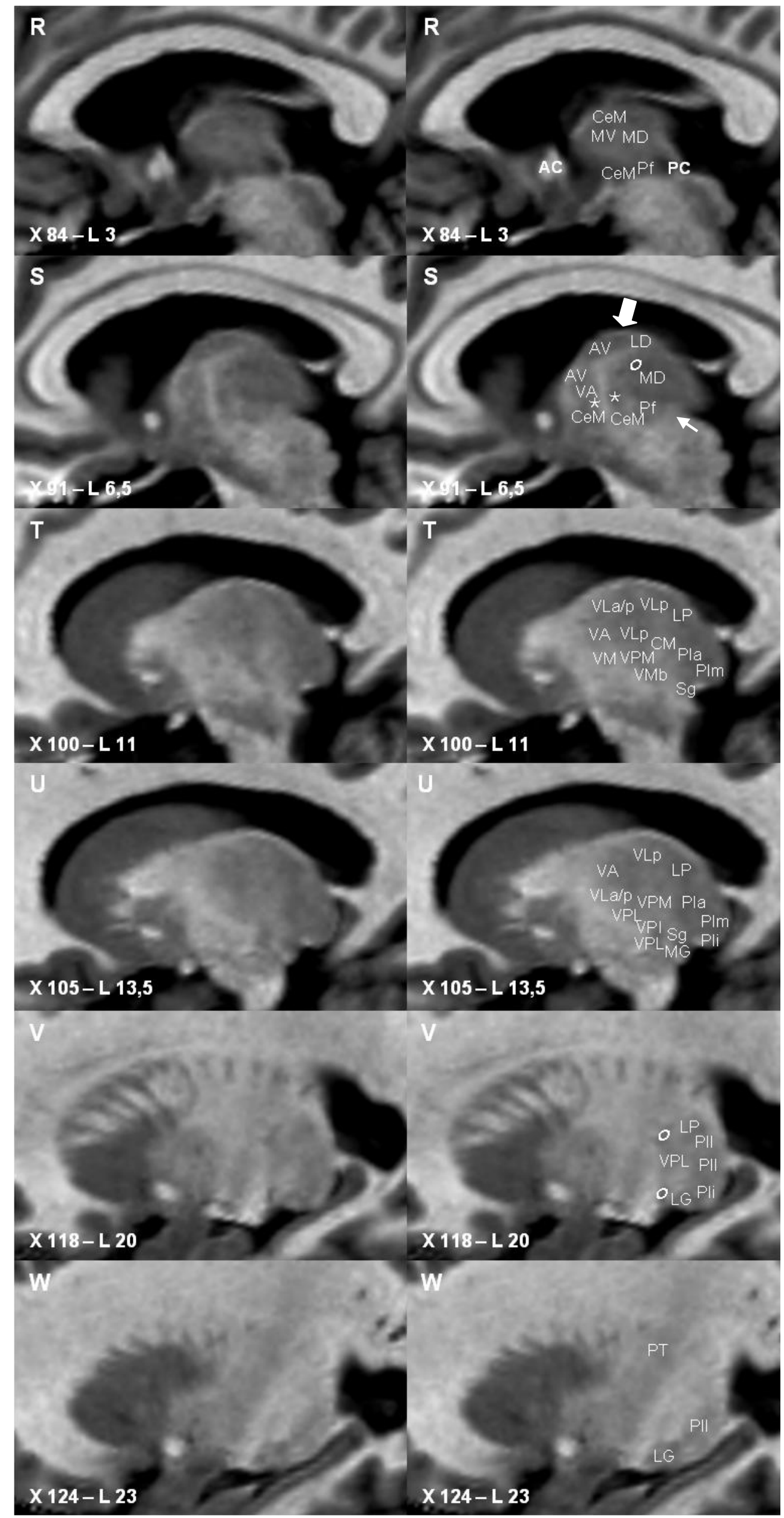

Figure 14: Sagittal panels

Continuous slices in medial to lateral order.

Orientated

perpendicular to $\mathrm{AC}$ PC level.

$\mathrm{X}$-coordinates derived from MRIcro.

Coordinates indicated in millimetres left (L) of origin at X 78 .

$\boldsymbol{O}$ : vessel

*: VAmc

: stria medullaris

$\boldsymbol{R}$ : fasciculus

retroflexus Meynertii 


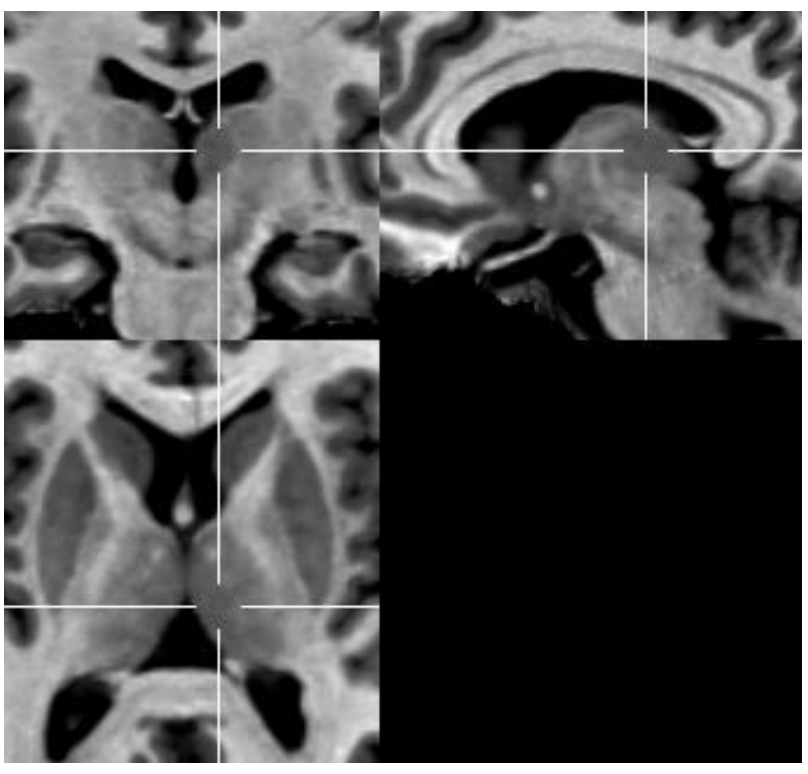

Figure 15: Mediodorsal nucleus - MD 1950-bg_MT-92-67-81

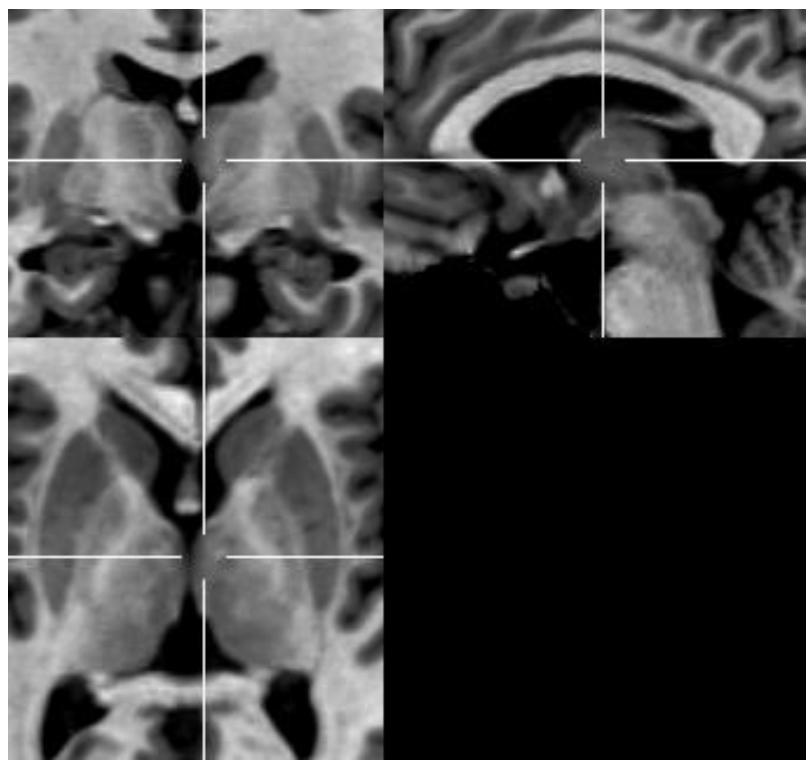

Figure 16: Medioventral nucleus- MV 1950-bg_MT-84-87-76

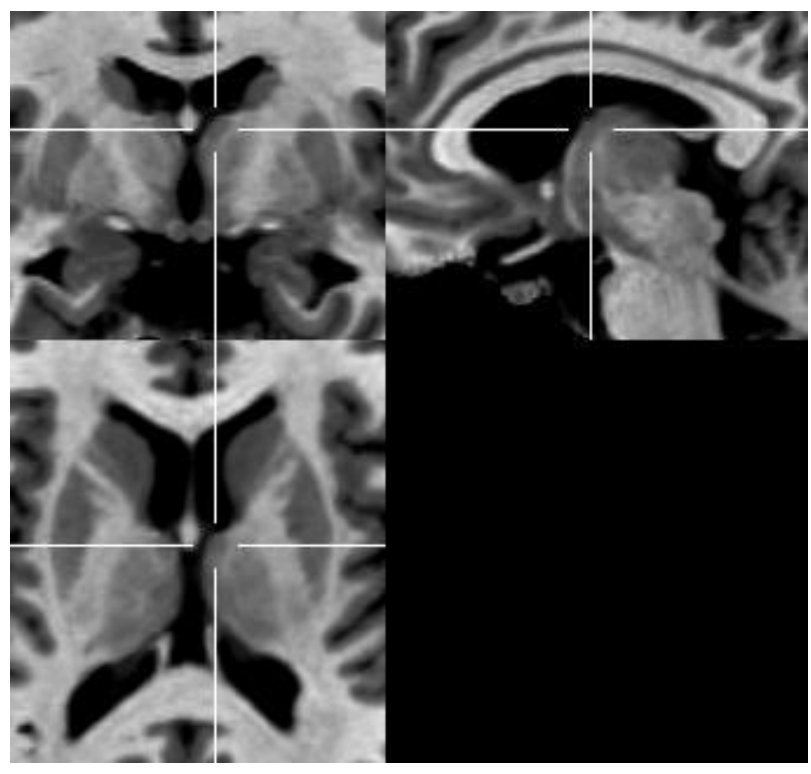

Figure 17: Anteroventral nucleus - AV

1950-bg_MT-88-93-90

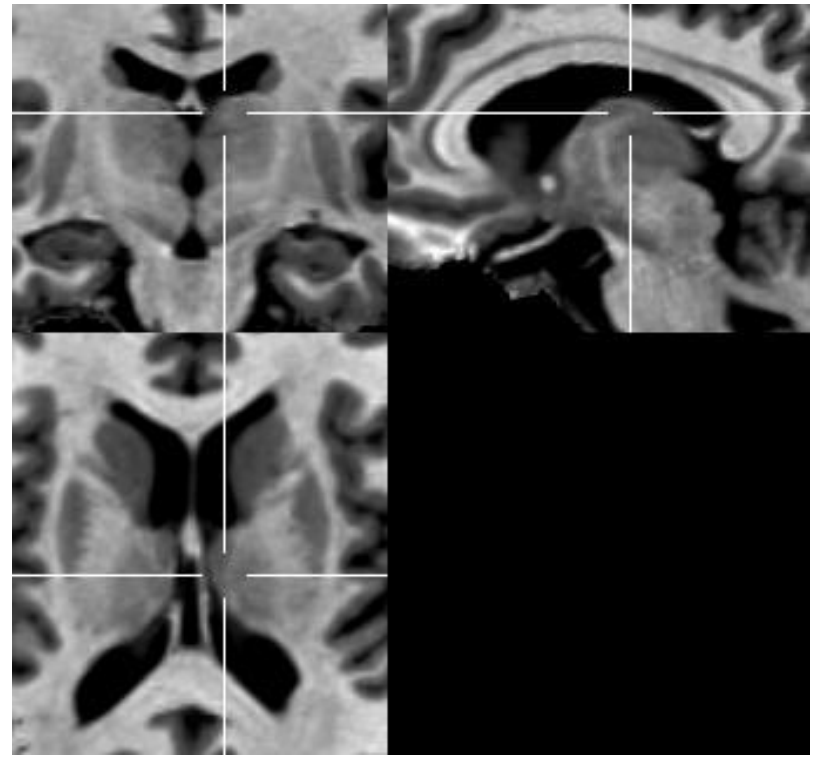

Figure 18: Lateral dorsal nucleus - LD 1950-bg_MT-91-77-94 


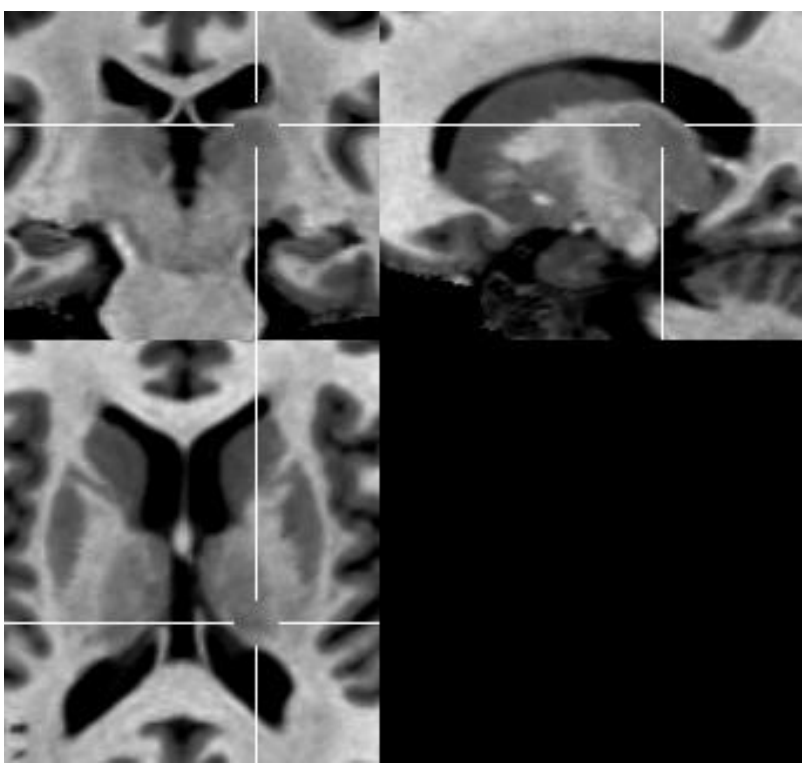

Figure 19: Lateral posterior nucleus - LP

1950-bg_MT-108-60-92

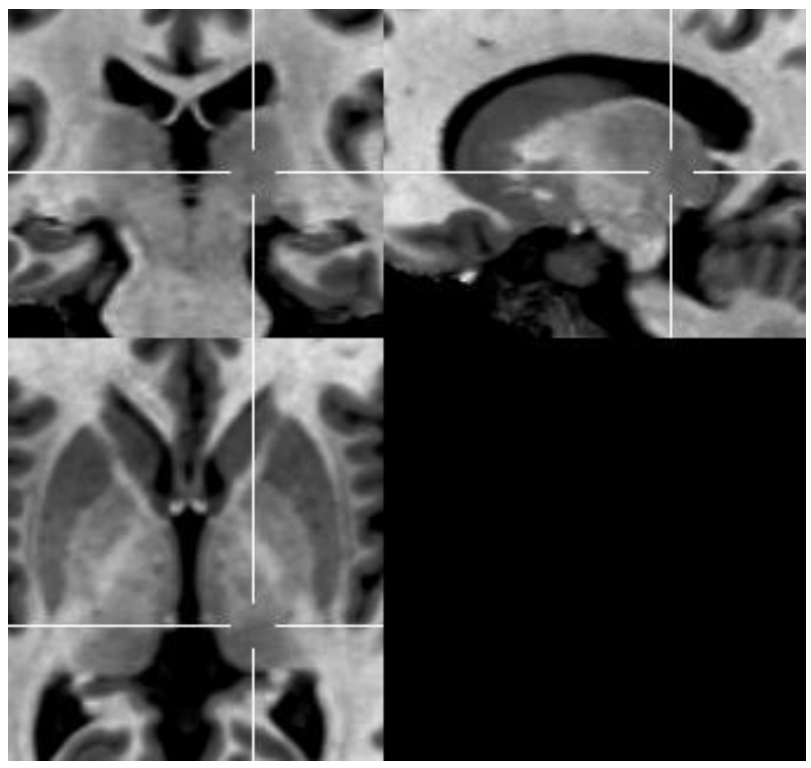

Figure 20: Anterior pulvinar nucleus - Pla 1950-bg_MT-105-58-71

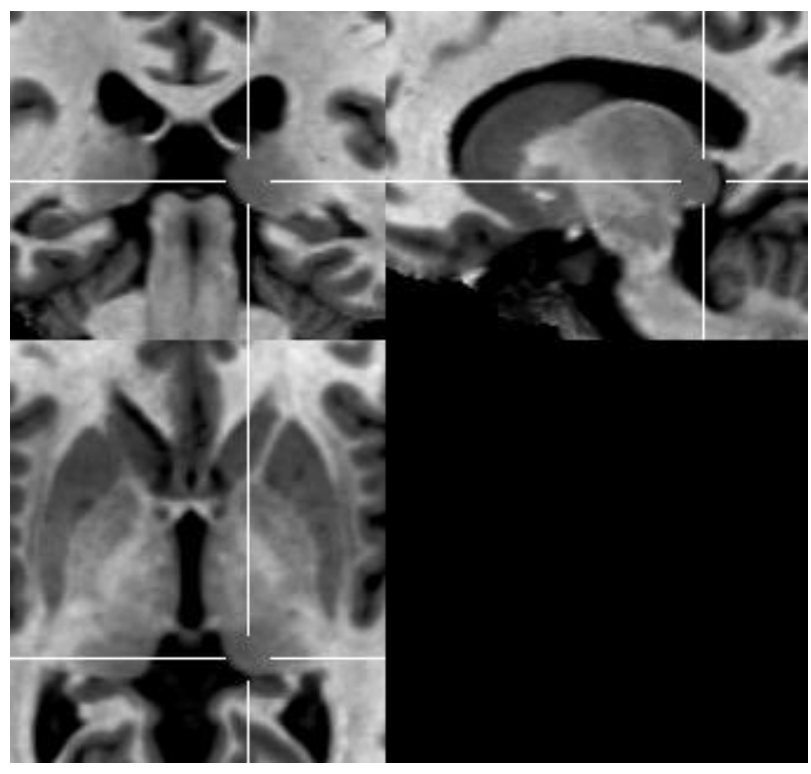

Figure 21: Medial pulvinar nucleus - PIm 1950-bg_MT-102-45-68

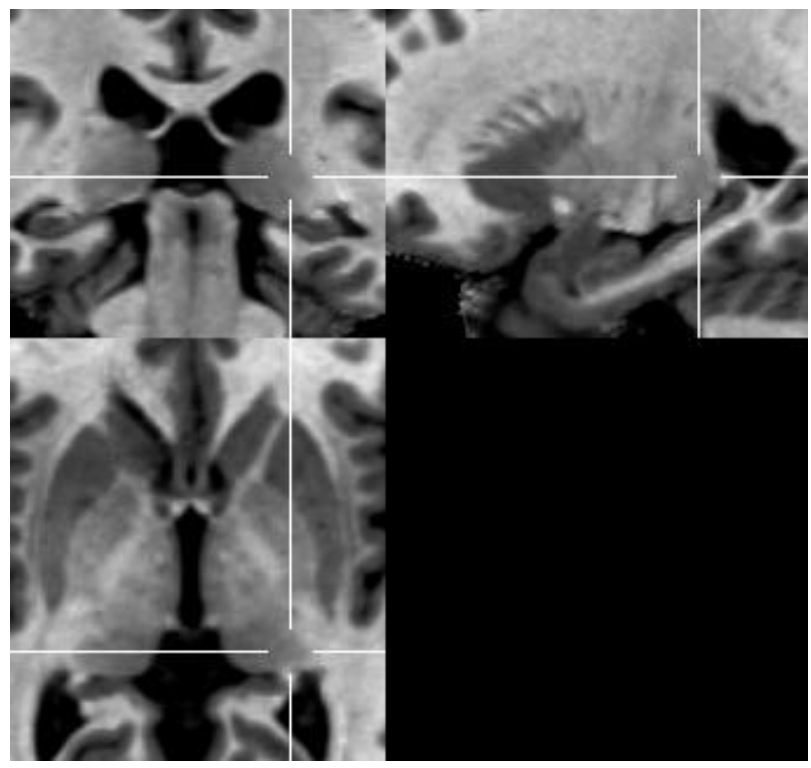

Figure 22: Lateral pulvinar nucleus - PIl 1950-bg_MT-120-47-69 


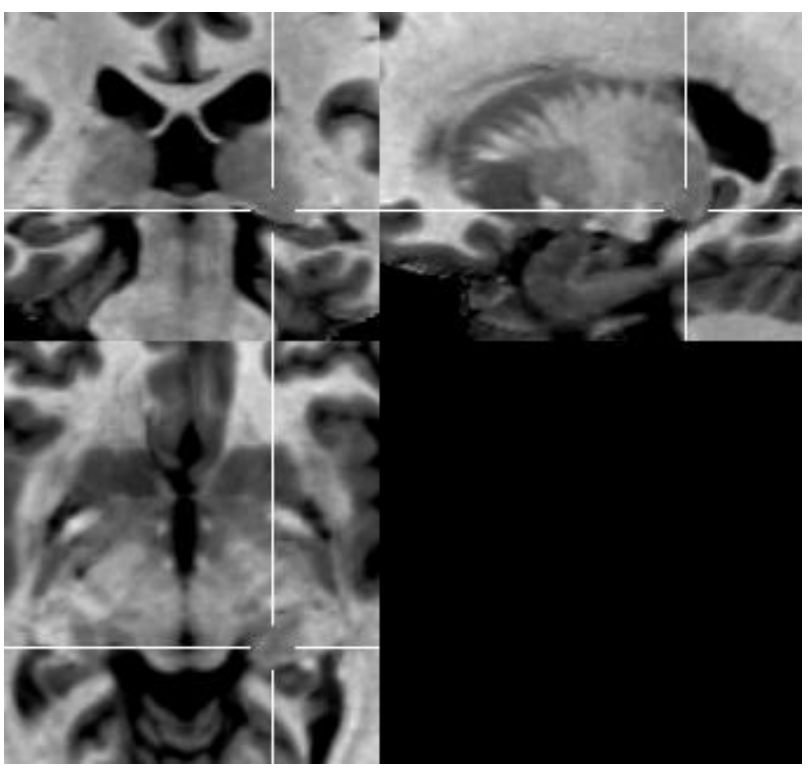

Figure 23: Inferior pulvinar nucleus - Pli

1950-bg_MT-115-50-56

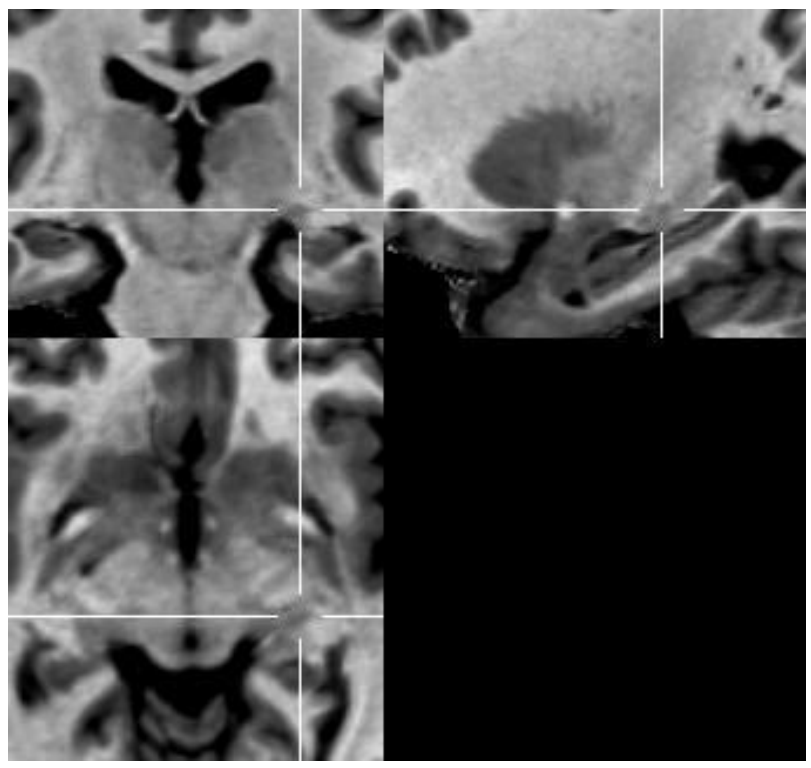

Figure 24: Lateral geniculate body - LG 1950-bg_MT-125-62-55

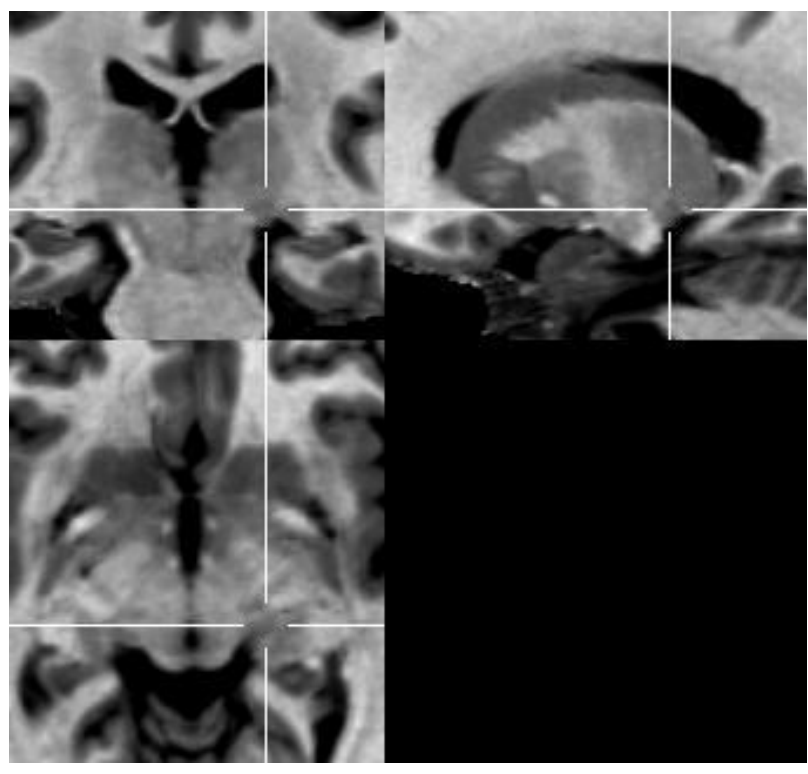

Figure 25: Medial geniculate body - MG 1950-bg_MT-110-59-56

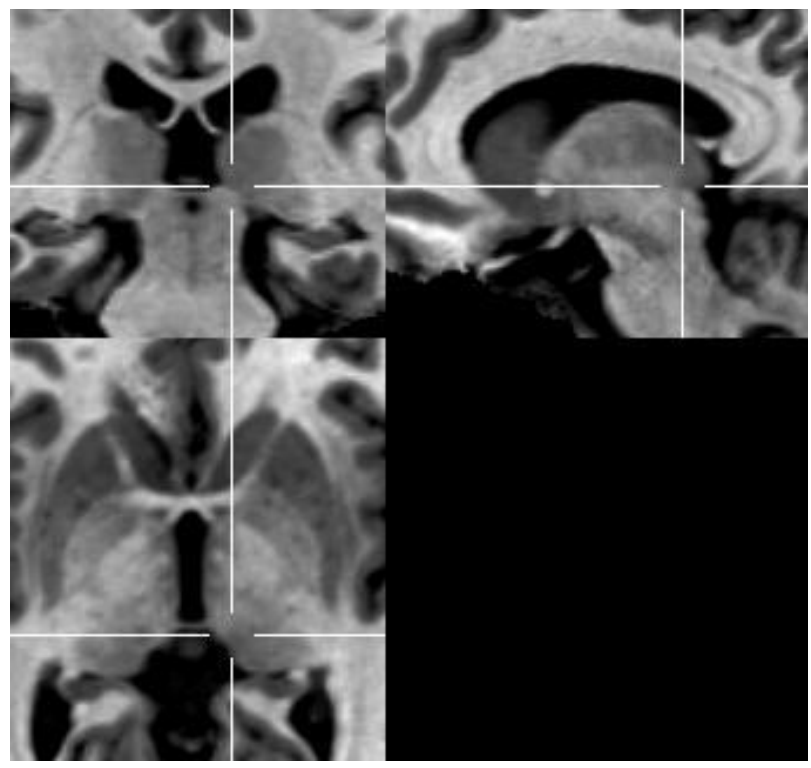

Figure 26: Limitans nucleus - L 1950-bg_MT-95-54-65 


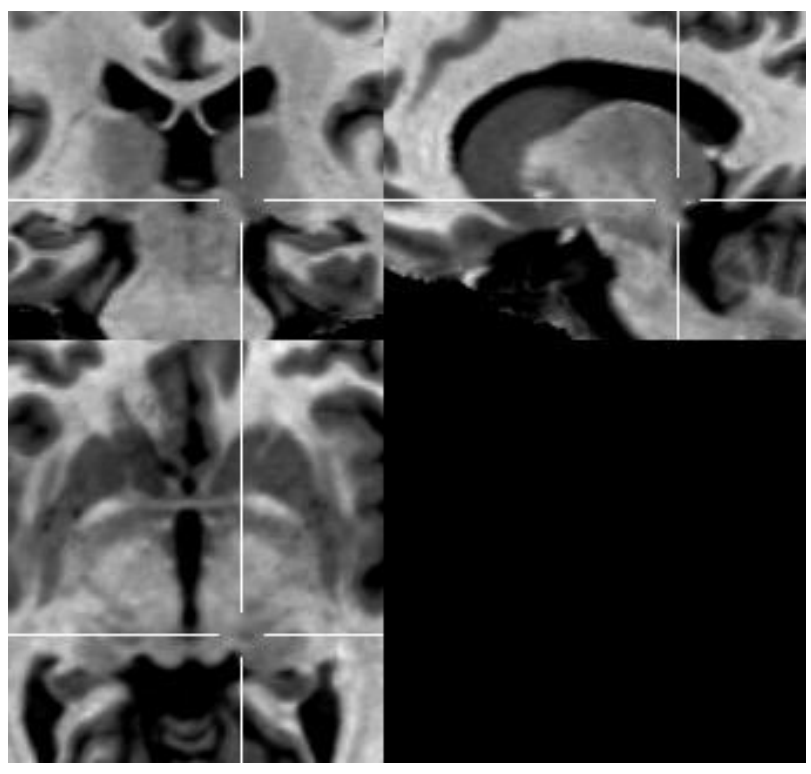

Figure 27: Suprageniculate nucleus - Sg

1950-bg_MT-100-55-60

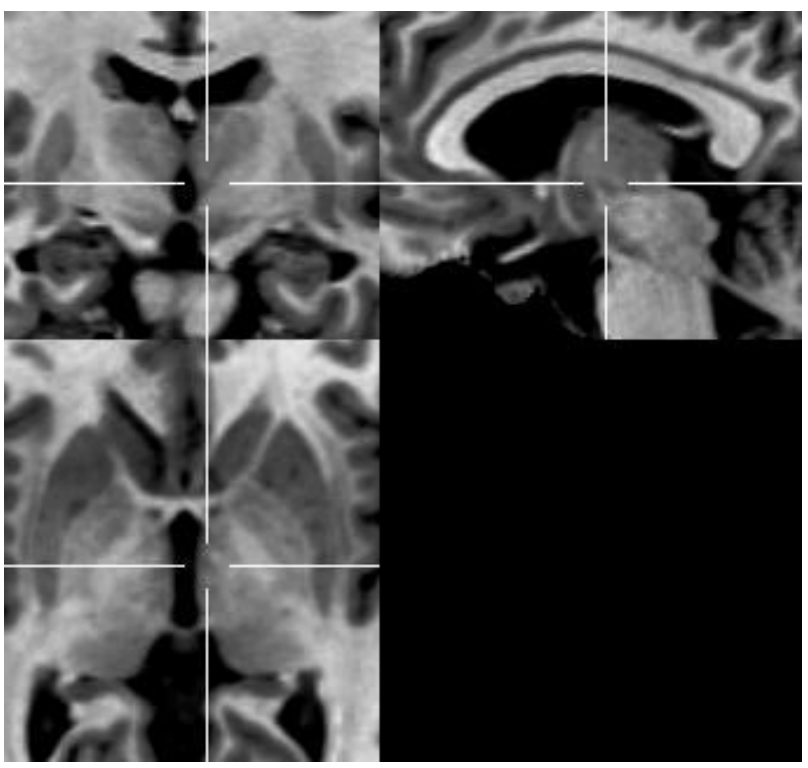

Figure 28: Central medial nucleus ventral part - CeM

1950-bg_MT-87-84-67

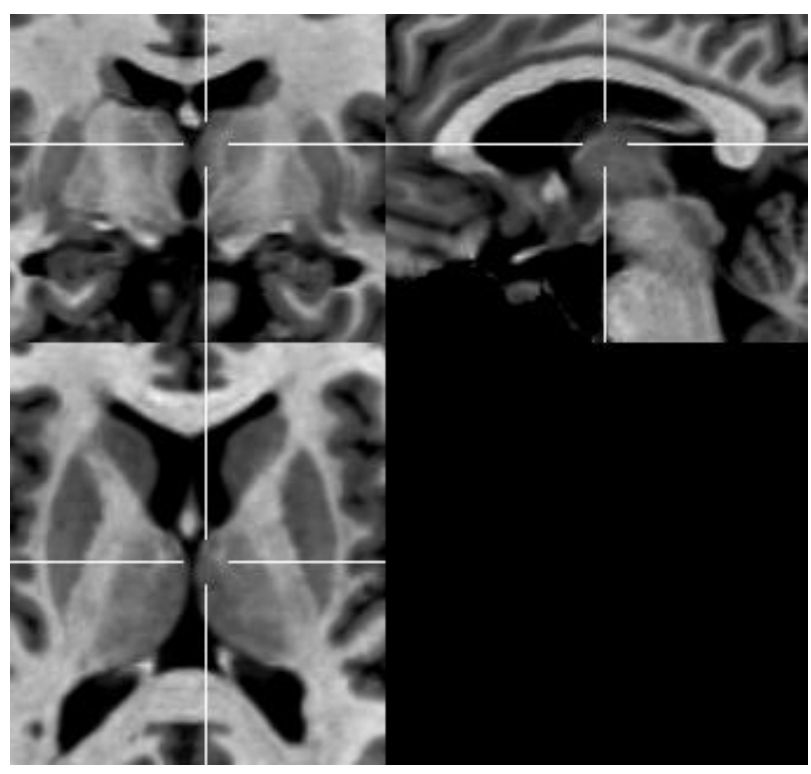

Figure 29: Central medial nucleus anterior part - CeM

1950-bg_MT-84-87-85 


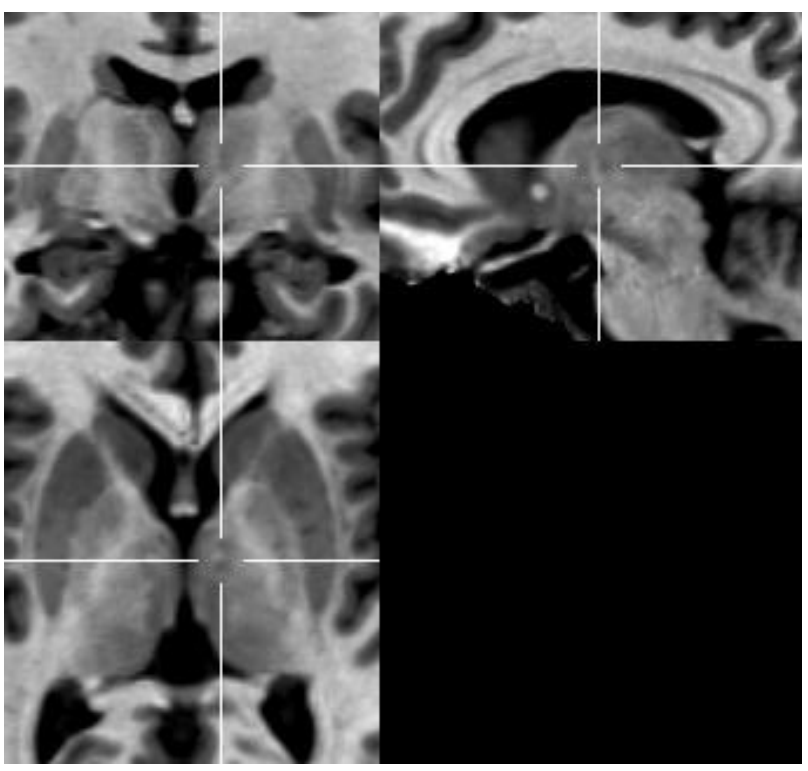

Figure 30: Magnocellular ventral anterior nucleus - VAmc

1950-bg_MT-93-87-75

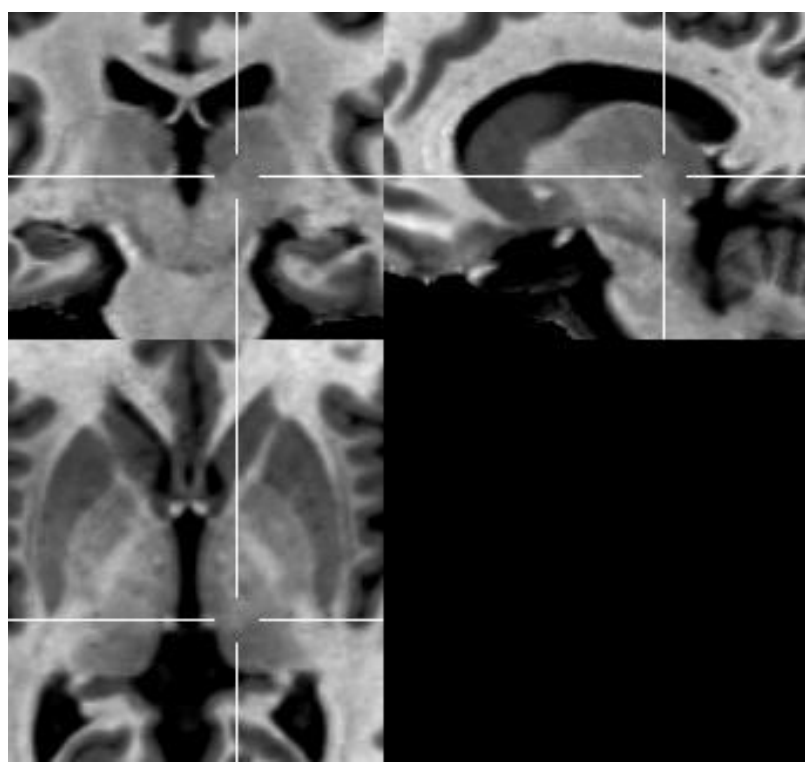

Figure 31: Centre médian nucleus - CM 1950-bg_MT-98-61-70

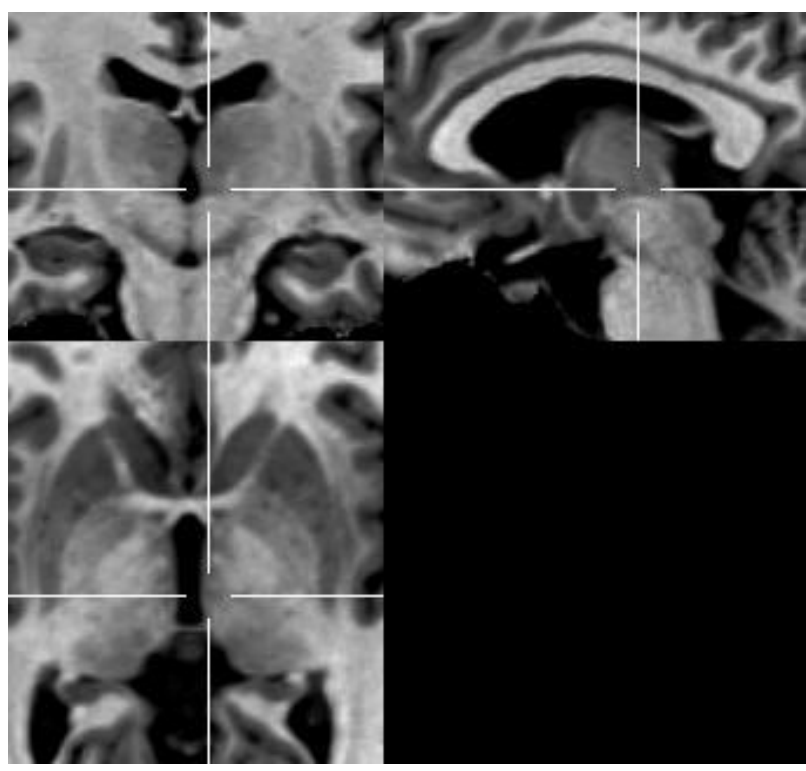

Figure 32: Parafascicular nucleus - Pf 1950-bg_MT-86-72-65

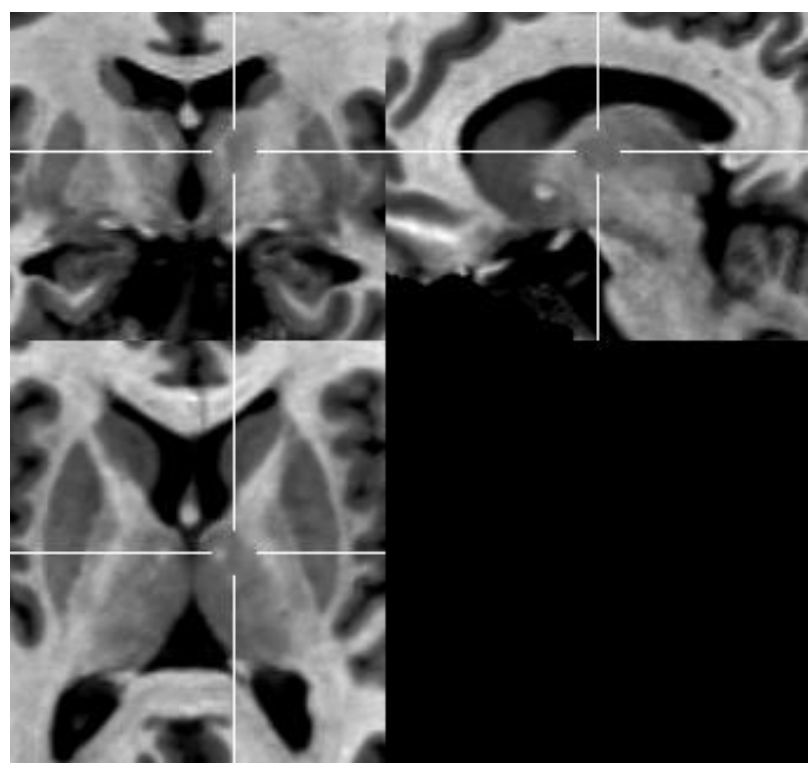

Figure 33: Ventral anterior nucleus - VA 1950-bg_MT-96-90-81 


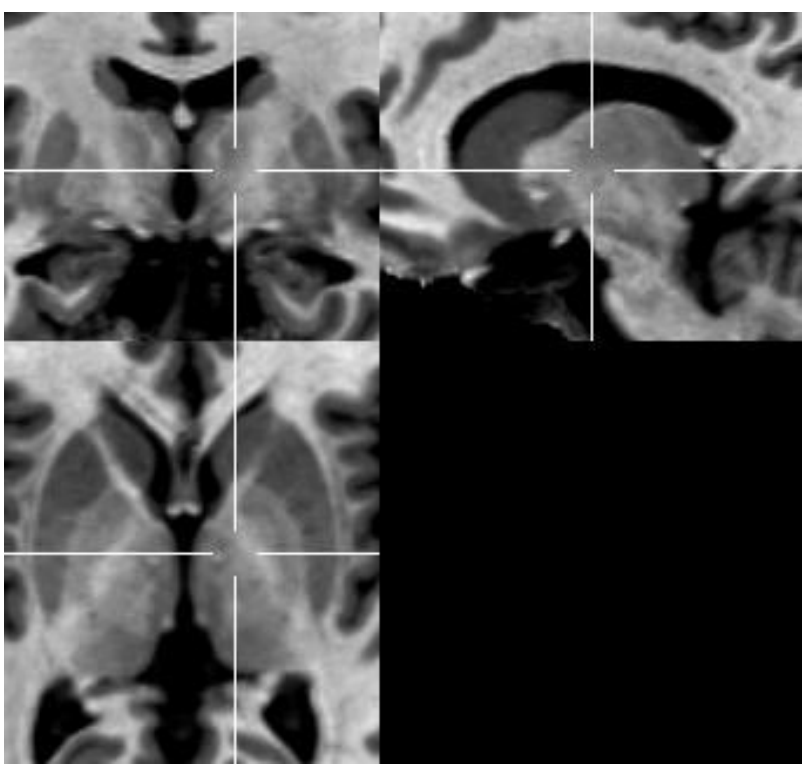

Figure 34: Principal ventral medial nucleus VM

1950-bg_MT-99-90-73

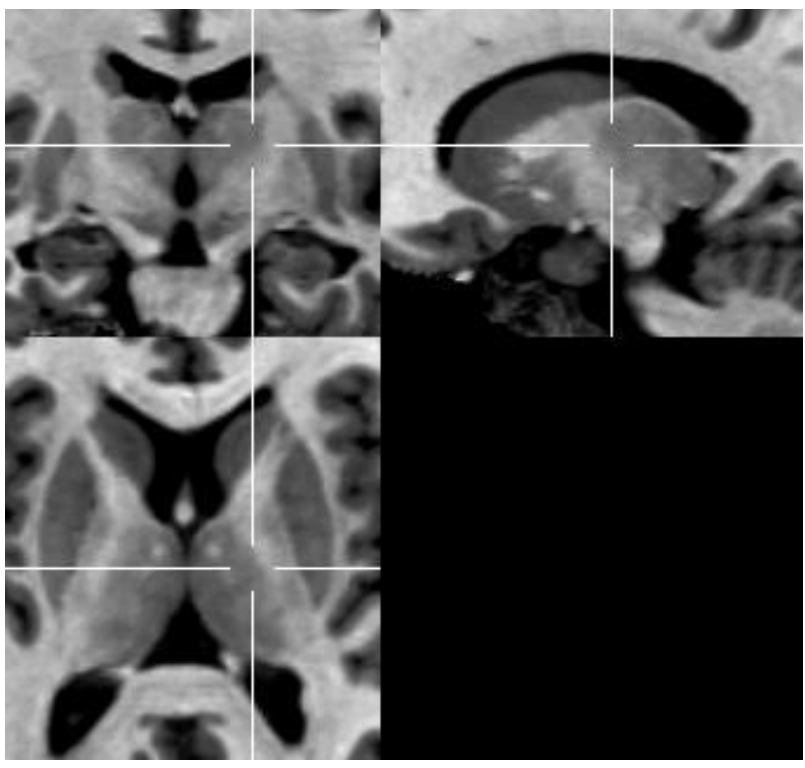

Figure 35: Ventral lateral anterior nucleus VLa

1950-bg_MT-106-82-82

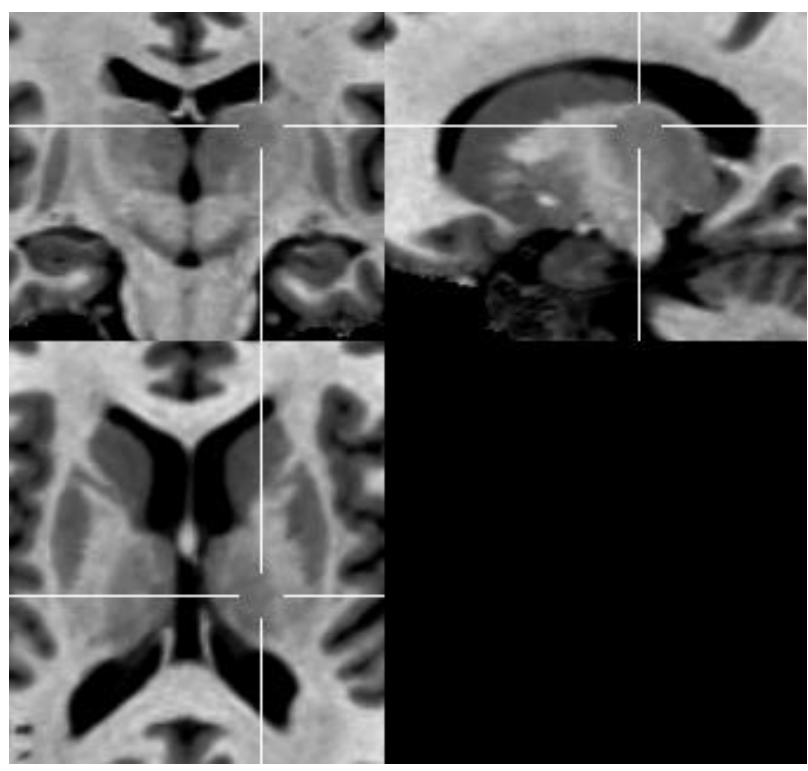

Figure 36: Ventral lateral posterior nucleus VLp

1950-bg_MT-108-72-92

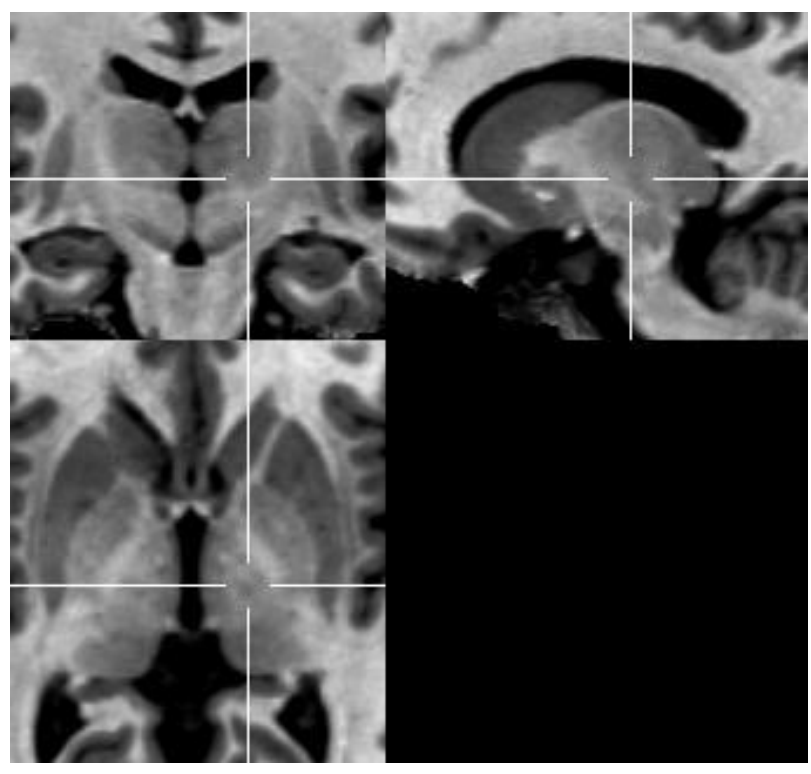

Figure 37: Ventral posterior medial nucleus/Ventral posterior lateral nucleus VPM/VPL

1950-bg_MT-102-76-69 


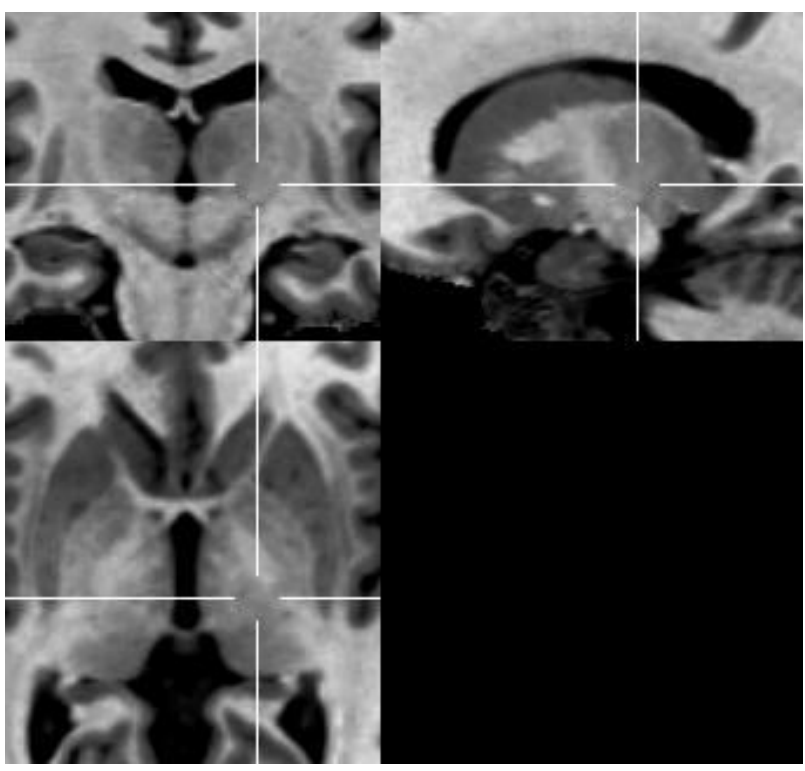

Figure 38: Ventral posterior inferior nucleus - VPI

1950-bg_MT-108-71-67

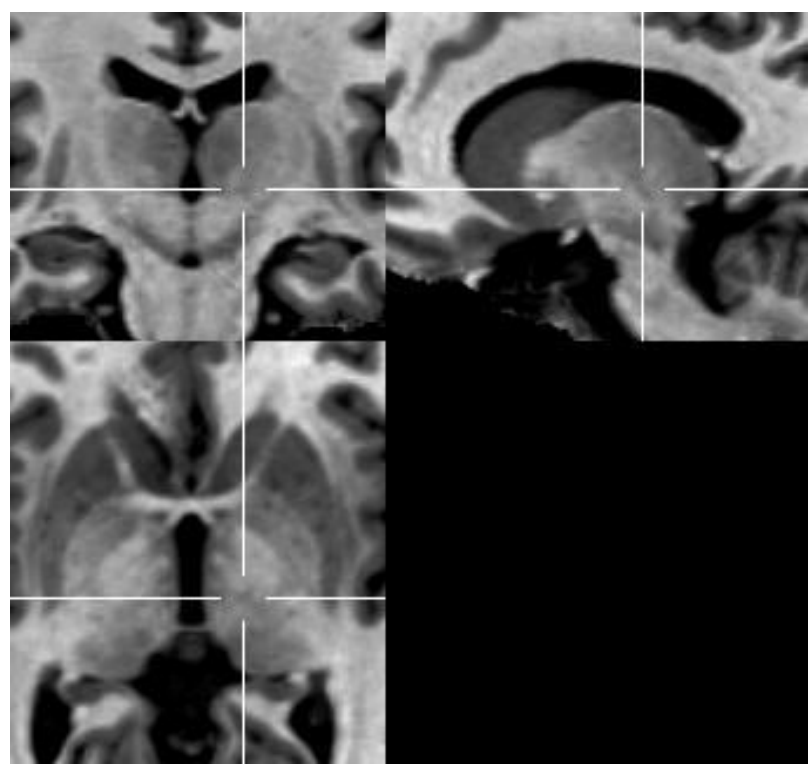

Figure 39: Basal ventral medial nucleus VMb

1950-bg_MT-100-71-65 


\subsection{LIST OF FIGURES}

Figure 1: Chart with the classification by Hirai and Jones (1989) ......................................... 9

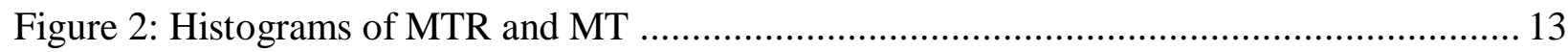

Figure 3: Calculation of MT saturation maps from MT-w, PD-w and T1-w FLASH images ..... 14

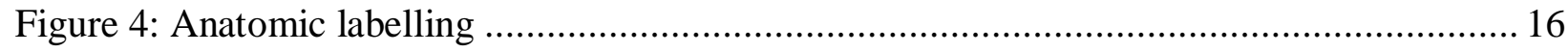

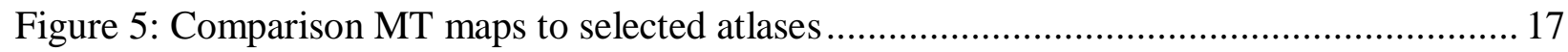

Figure 6: Overlay of camera lucida drawings on MT and R2* maps - coronal ....................... 18

Figure 7: Overlay of camera lucida drawings on MT and R2* maps - sagittal ....................... 19

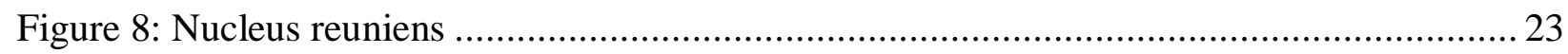

Figure 9: Hypothetical position of the V.im ................................................................. 41

Figure 10: MT map resembling a histological section .............................................. 42

Figure 11: Orthogonal display of hum_1950 at coordinates $65-89-91 \ldots \ldots \ldots \ldots \ldots \ldots \ldots \ldots \ldots \ldots \ldots \ldots . . . . . . \ldots \ldots$

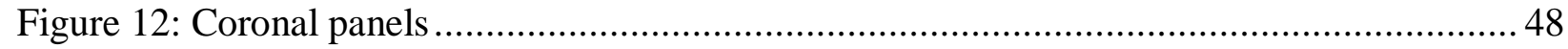

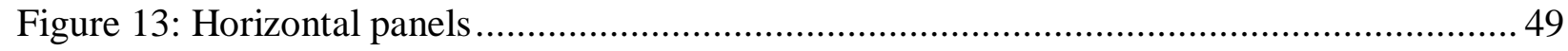

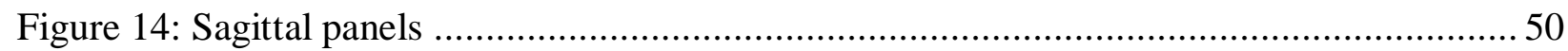

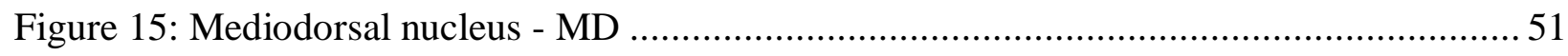

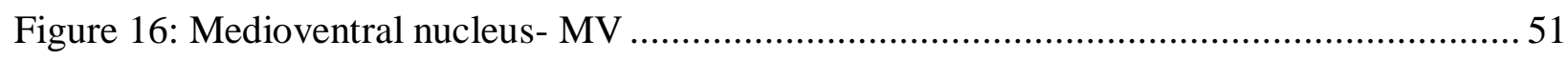

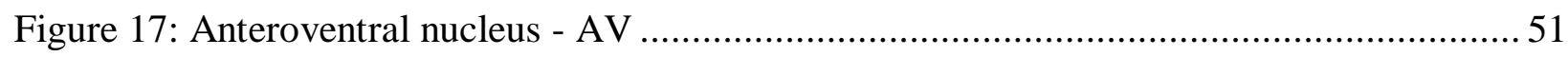

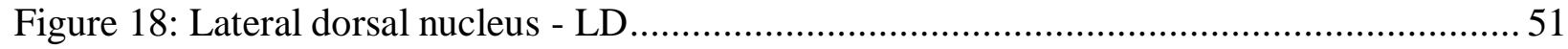

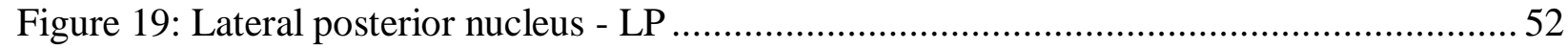

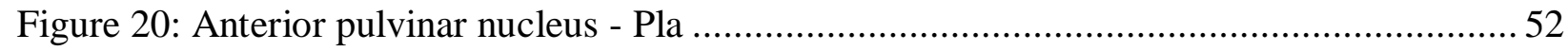

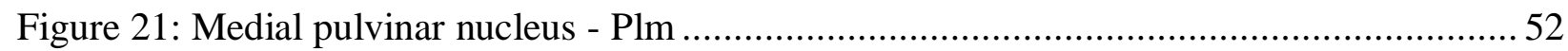

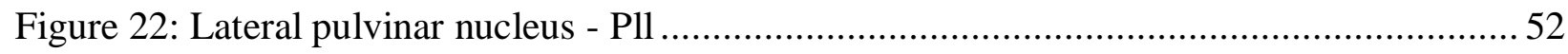

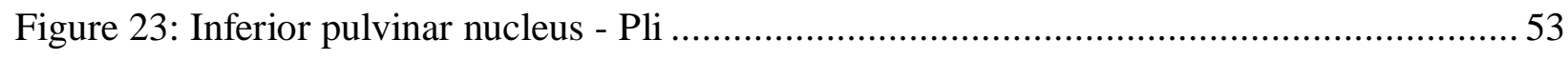

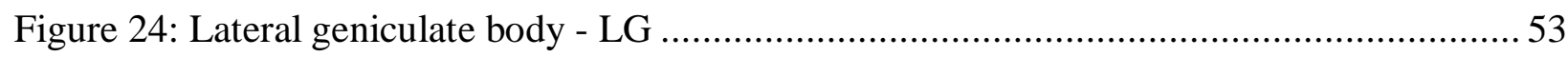

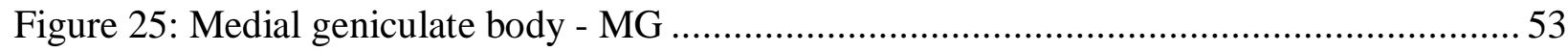

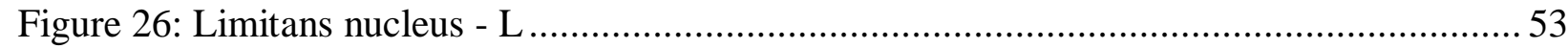

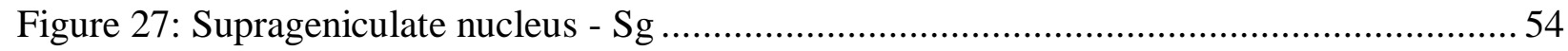

Figure 28: Central medial nucleus ventral part - CeM ................................................... 54

Figure 29: Central medial nucleus anterior part - CeM...................................................... 54

Figure 30: Magnocellular ventral anterior nucleus - VAmc ............................................... 55

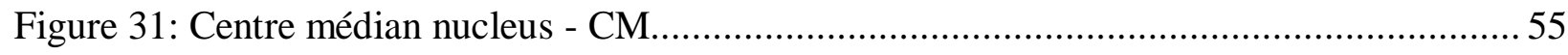

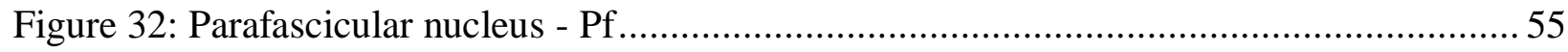

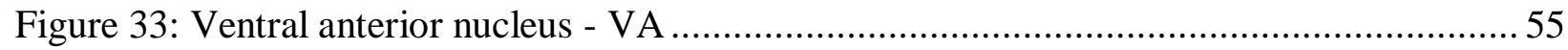


Figure 34: Principal ventral medial nucleus - VM.............................................................. 56

Figure 35: Ventral lateral anterior nucleus - VLa ........................................................... 56

Figure 36: Ventral lateral posterior nucleus - VLp...................................................... 56

Figure 37: Ventral posterior medial nucleus/Ventral posterior lateral nucleus - VPM/VPL....... 56

Figure 38: Ventral posterior inferior nucleus - VPI ....................................................... 57

Figure 39: Basal ventral medial nucleus - VMb ............................................................... 57

\subsection{Publications Related to this Dissertation}

\subsubsection{PAPERS}

Elolf E, Bockermann V, Gringel T, Knauth M, Dechent P, Helms G

Improved visibility of the subthalamic nucleus by added susceptibility (T2*) contrast using multiple gradient-echoes.

AJNR Am J Neuroradiol 2007;28(5)1093-7

Gringel T, Schulz-Schaeffer W, Elolf E, Frölich A, Dechent P, Helms G

Optimized high-resolution mapping of magnetization transfer (MT) at 3 Tesla for direct visualization of substructures of the human thalamus in clinically feasible measurement time.

J Magn Reson Imaging 2009 Jun;29(6):1285-92

\subsubsection{Presentations / Peer Reviewed Abstracts}

Gringel T, Elolf E, Schulz-Schaeffer W, Dechent P, Knauth M, Helms G

Identifizierung thalamischer Substrukturen bei 3 T auf kontrastoptimierten Parameterkarten.

88. Deutscher Röntgenkongress, Berlin, 16.-19. Mai 2007

Abstract in: Fortschr Röntgenstr 2007; 179S. DOI: 10.1055/s-2007-977116

Helms G, Gringel T, Elolf E, Schulz-Schaeffer W, Dechent P

Optimized high-resolution mapping of magnetisation transfer at 3 Tesla reveals substructures in the human thalamus in clinically feasible measurement time.

Joint Annual Meeting of the ISMRM and ESMRMB, Berlin, Germany, 19-25 May 2007

Abstract in: Proc Intl Soc Magn Reson Med (2007) 14:2058 
Elolf E, Gringel T, Dechent P, Knauth M, Helms G

Systematische Identifizierung der Substrukturen des Thalamus auf kontrast-optimierten Parameterkarten bei 3 Tesla.

42. Jahrestagung der Deutschen Gesellschaft für Neuroradiologie, Frankfurt am Main, 29. August - 01. September 2007

Abstract in: Clin Neuroradiol 2007;17 (Spec Issue 1):14

Elolf E, Gringel T, Dechent P, Helms G, Knauth M

Visualisierung des STN (Nucleus subthalamicus) auf R2*-Karten an einem klinischen 3-TeslaMRT-System.

42. Jahrestagung der Deutschen Gesellschaft für Neuroradiologie, Frankfurt am Main, 29. August - 01. September 2007

Abstract in: Clin Neuroradiol 2007;17 (Spec Issue 1):14

Helms G, Gringel T, Knauth M, Dechent P, Elolf E.

Delineation of the subthalamic nucleus (STN) on high-resolution maps of R2*.

16th Annual Meeting of the ISMRM, Toronto, Canada, 5-11 May 2008

Abstract in: Proc Intl Soc Mag Reson Med (2008) 16:880

Dechent P, Gringel T, Elolf E, Schulz-Schaeffer W, Helms G

Optimized high-resolution mapping of magnetisation transfer at 3 Tesla reveals substructures in the human thalamus in clinically feasible measurement time.

$14^{\text {th }}$ Annual Meeting of the Organization for Human Brain Mapping, Melbourne, Australia, 1519 June 2008

Abstract in: Neuroimage 41(S1) 2008

Dechent P, Elolf E, Gringel T, Knauth M, Helms G.

Delineation of the subthalamic nucleus (STN) on high-resolution maps of $R 2$ *.

$14^{\text {th }}$ Annual Meeting of the Organization for Human Brain Mapping, Melbourne, Australia, 1519 June 2008

Abstract in: Neuroimage 41(S1) 2008 


\section{REFERENCES}

Abosch A, Yacoub E, Ugurbil K, Harel N (2010): An assessment of current brain targets for deep brain stimulation surgery with susceptibility-weighted imaging at 7 Tesla. Neurosurgery 67(6), 1745-1756

Bähr M, Duus P, Frotscher M, Küker W: Duus' Neurologisch-topische Diagnostik Anatomie, Funktion, Klinik. 8. komplett überarb. Aufl.; Thieme, Stuttgart 2003

Behrens TE, Johansen-Berg H, Woolrich MW, Smith SM, Wheeler-Kingshott CA, Boulby PA, Barker GJ, Sillery EL, Sheehan K, Ciccarelli O, et al. (2003): Non-invasive mapping of connections between human thalamus and cortex using diffusion imaging. Nat Neurosci $\underline{6}(7), 750-757$

Ben-Haim S, Asaad WF, Gale JT, Eskandar EN (2009): Risk Factors for Hemorrhage During Microelectrode-Guided Deep Brain Stimulation and the Introduction of An Improved Microelectrode Design. Neurosurgery 64(4), 754-763

Benabid AL, Pollak P, Gao D, Hoffmann D, Limousin P, Gay E, Payen I, Benazzouz A (1996): Chronic electrical stimulation of the ventralis intermedius nucleus of the thalamus as a treatment of movement disorders. J Neurosurg 84(2), 203-214

Benabid AL, Koudsie A, Benazzouz A, Le Bas JF, Pollak P (2002): Imaging of subthalamic nucleus and ventralis intermedius of the thalamus. Mov Disord 17 Suppl 3, 123-129

Bender B, Mänz C, Korn A, Nägele T, Klose U (2011): Optimized 3D Magnetization-Prepared Rapid Acquisition of Gradient Echo: Identification of Thalamus Substructures at 3T. AJNR Am J Neuroradiol 32(11), 2110-2115

Bogen JE (2006): A half-century of perpetuating Netter's anatomic error. J Hist Neurosci 15(1), 53-55

Briggs F, Usrey WM (2008): Emerging views of corticothalamic function. Curr Opin Neurobiol $\underline{18}(4), 403-407$

Burdach KF: Vom Baue und Leben des Gehirns. Erster Band. Dyksche Buchhandlung, Leipzig 1819

Burdach KF: Vom Baue und Leben des Gehirns. Zweyter Band. Dyksche Buchhandlung, Leipzig 1822

Coenen V, Prescher A, Schmidt T, Picozzi P, Gielen F (2008): What is dorso-lateral in the subthalamic Nucleus (STN)? - a topographic and anatomical consideration on the ambiguous description of today's primary target for deep brain stimulation (DBS) surgery. Acta Neurochir (Wien) 150(11), 1163-1165

Deoni SC, Josseau MJ, Rutt BK, Peters TM (2005): Visualization of thalamic nuclei on high resolution, multi-averaged T1 and T2 maps acquired at 1.5 T. Hum Brain Mapp 25(3), 353-359

Deoni SCL, Rutt BK, Parrent AG, Peters TM (2007): Segmentation of thalamic nuclei using a modified k-means clustering algorithm and high-resolution quantitative magnetic resonance imaging at $1.5 \mathrm{~T}$. Neuroimage $\underline{34}(1), 117-126$

Dewulf A: Anatomy of the normal human thalamus: topometry and standardized nomenclature. Elsevier, Amsterdam 1971

Dousset V, Grossman RI, Ramer KN, Schnall MD, Young LH, Gonzalez-Scarano F, Lavi E, Cohen JA (1992): Experimental allergic encephalomyelitis and multiple sclerosis: lesion characterization with magnetization transfer imaging. Radiology 182(2), 483-491 
Edzes HT, Samulski ET (1977): Cross relaxation and spin diffusion in the proton NMR of hydrated collagen. Nature 265(5594), 521-523

Elolf E, Bockermann V, Gringel T, Knauth M, Dechent P, Helms G (2007): Improved visibility of the subthalamic nucleus on high-resolution stereotactic MR imaging by added susceptibility $(\mathrm{T} 2 *)$ contrast using multiple gradient echoes. AJNR Am J Neuroradiol 28(6), 1093-1094

Fisher R, Salanova V, Witt T, Worth R, Henry T, Gross R, Oommen K, Osorio I, Nazzaro J, Labar D, et al. (2010): Electrical stimulation of the anterior nucleus of thalamus for treatment of refractory epilepsy. Epilepsia 51(5), 899-908

Gallay MN, Jeanmonod D, Liu J, Morel A (2008): Human pallidothalamic and cerebellothalamic tracts: anatomical basis for functional stereotactic neurosurgery. Brain Struct Funct $\underline{212}(6), 443-463$

Gelman N, Ewing J, Gorell J, Spickler E, Solomon E (2001): Interregional variation of longitudinal relaxation in human brain at 3.0 T: relation to iron and water content. Magn Reson Med 45(1), 71-79

Giudicelli G, Salamon G (1970): The veins of the thalamus. Neuroradiology 1, 92-98

Gringel T, Schulz-Schaeffer WJ, Elolf E, Dechent P, Helms G (2009): Optimized highresolution mapping of magnetization transfer (MT) at 3 Tesla for direct visualization of substructures of the human thalamus in clinically feasible measurement time. J Magn Reson Imaging 29(6), 1285-1292

Guillery RW (2005): Anatomical pathways that link perception and action. Prog Brain Res $\underline{149}$, 235-256

Hassler R, Riechert T (1954): Indikationen und Lokalisationsmethode der gezielten Hirnoperationen. Nervenarzt 20;25(11), 441-447

Hassler R: Anatomy of the Thalamus. In: G. Schaltenbrand and P. Bailey (Eds.) Introduction to Stereotaxis with an Atlas of the Human Brain. Thieme, Stuttgart 1959, pp. 230-290

Helms G, Piringer A (2005): Simultaneous measurement of saturation and relaxation in human brain by repetitive magnetization transfer pulses. NMR Biomed 18 11 , 44-50

Helms G (2006): Interaction of exchange and differential relaxation in the saturation recovery behavior of the binary spin-bath model for magnetization transfer. Concepts Magn Reson $\underline{28 \mathrm{~A}}(4), 291-298$

Helms G, Gringel T, Elolf E, Schulz-Schaeffer WJ, Dechent P: Optimized high-resolution mapping of magnetisation transfer at 3 Tesla reveals substructures in the human thalamus in clinically feasible measurement time. Joint Annual Meeting of the ISMRM and ESMRMB, Berlin, 19 - 25 May 2007, Abstract in: Proc Intl Soc Magn Reson Med, $14: 2058$

Helms G, Gringel T, Knauth M, Dechent P, Elolf E: Delineation of the subthalamic nucleus (STN) on high-resolution maps of R2*. 16th Annual Scientific Meeting of ISMRM, Toronto, ON, Canada, 5 - 11 May 2008a, Abstract in: Proc Intl Soc Magn Reson Med, $16: 880$

Helms G, Dathe H, Dechent P (2008b): Quantitative FLASH MRI at 3T using a rational approximation of the Ernst equation. Magn Reson Med 59(3), 667-672

Helms G, Dathe H, Kallenberg K, Dechent P (2008c): High-resolution maps of magnetization transfer with inherent correction for RF inhomogeneity and $\mathrm{T} 1$ relaxation obtained from 3D FLASH MRI. Magn Reson Med 60(6), 1396-1407

Helms G, Dechent P (2009): Increased SNR and reduced distortions by averaging multiple gradient echo signals in 3D FLASH imaging of the human brain at 3T. J Magn Reson Imaging 29(1), 198-204

Helms G, Draganski B, Frackowiak R, Ashburner J, Weiskopf N (2009a): Reliable segmentation of deep brain grey matter structures using magnetization transfer (MT) parameter maps. Neuroimage 47 (1), 194-198 
Helms G, Draganski B, Frackowiak R, Ashburner J, Weiskopf N (2009b): Improved segmentation of deep brain grey matter structures using magnetization transfer (MT) parameter maps. Neuroimage 47(1), 194-198

Helms G, Dathe H, Dechent P (2010): Modeling the influence of TR and excitation flip angle on the magnetization transfer ratio (MTR) in human brain obtained from 3D spoiled gradient echo MRI. Magn Reson Med 64(1), 177-185

Henkelman RM, Stanisz GJ, Graham SJ (2001): Magnetization transfer in MRI: a review. NMR Biomed 14(2), 57-64

Hirai T, Jones EG (1989): A new parcellation of the human thalamus on the basis of histochemical staining. Brain Res Brain Res Rev 14(1), 1-34

Jbabdi S, Woolrich MW, Behrens TE (2009): Multiple-subjects connectivity-based parcellation using hierarchical Dirichlet process mixture models. Neuroimage 44(2), 373-384

Jones EG: The Thalamus. Plenum Press, New York 1985

Jones EG: The Thalamus. 2nd ed.; Cambridge University Press, Cambridge 2007

Kamman RL, Go KG, Brouwer W, Berendsen HJC (1988): Nuclear magnetic resonance relaxation in experimental brain edema: Effects of water concentration, protein concentration, and temperature. Magn Reson Med 6(3), 265-274

Kandel ER, Schwartz JH, Jessell TM: Principles of neural science. 4th ed.; McGraw-Hill, Health Professions Division, New York; London 2000

Klein J, Rushworth M, Behrens T, Mackay C, de Crespigny A, D'Arceuil H, Johansen-Berg H (2010): Topography of connections between human prefrontal cortex and mediodorsal thalamus studied with diffusion tractography. Neuroimage 51(2), 555-564

Krause M, Fogel W, Heck A, Hacke W, Bonsanto M, Trenkwalder C, Tronnier V (2001): Deep brain stimulation for the treatment of Parkinson's disease: subthalamic nucleus versus globus pallidus internus. J Neurol Neurosurg Psychiatry 70(4), 464-470

Krauth A, Blanc R, Poveda A, Jeanmonod D, Morel A, Székely G (2010): A mean threedimensional atlas of the human thalamus: Generation from multiple histological data. Neuroimage 49(3), 2053-2062

Le Gros Clark WE (1932): The structure and connections of the thalamus. Brain 55(3), 406-470

Luys J: Recherches sur le système cérébro-spinal, sa structure, ses fonctions et ses maladies. Ballière, Paris 1865

Magnotta VA, Gold S, Andreasen NC, Ehrhardt JC, Yuh WT (2000): Visualization of subthalamic nuclei with cortex attenuated inversion recovery MR imaging. Neuroimage 11(4), 341-346

Mai JK, Paxinos G, Voss T: Altas of the Human Brain. 3; Academic Press, New York 2008

Mang SC, Busza A, Reiterer S, Grodd W, Klose U (2011): Thalamus segmentation based on the local diffusion direction: A group study. Magn Reson Med 67(1), 118-126

Martin JH: Neuroanatomy : text and atlas. 2nd ed.; Prentice Hall, London 1996

Morel A, Magnin M, Jeanmonod D (1997): Multiarchitectonic and stereotactic atlas of the human thalamus. J Comp Neurol 387(4), 588-630

Niemann K, Naujokat C, Pohl G, Wollner C, von Keyserlingk D (1994): Verification of the Schaltenbrand and Wahren stereotactic atlas. Acta Neurochir (Wien) 129(1-2), 72-81

Niemann K, van Nieuwenhofen I (1999): One atlas - three anatomies: relationships of the Schaltenbrand and Wahren microscopic data. Acta Neurochir (Wien) 141(10), 1025-1038

Niemann K, Mennicken VR, Jeanmonod D, Morel A (2000): The Morel stereotactic atlas of the human thalamus: atlas-to-MR registration of internally consistent canonical model. Neuroimage 12(6), 601-616

Nissl F (1913): Die Grosshirnanteile des Kaninchens. Eur Arch Psychiatry Clin Neurosci 52(3), $867-953$

Olszewski J: The Thalamus of the Macaca mulatta. An Atlas for use with the Stereotaxic Instrument. Karger, Basel 1952 
Otsuki T, Jokura H, Takahashi K, Ishikawa S, Yoshimoto T, Kimura M, Yoshida R, Miyazawa T (1994): Stereotactic Thalamotomy with a Computerized Brain Atlas: Technical Case Report. Neurosurgery 35(4), 764-768

Percheron G, Yelnik J, François C (1986): Systems of coordinates for stereotactic surgery and cerebral cartography: advantages of ventricular systems in monkeys. J Neurosci Methods $17(2-3), 69-88$

Pinsker M, Volkmann J, Falk D, Herzog J, Steigerwald F, Deuschl G, Mehdorn H (2009): Deep brain stimulation of the internal globus pallidus in dystonia: target localisation under general anaesthesia. Acta Neurochir (Wien) 151(7), 751-758

Pschyrembel W: Klinisches Wörterbuch. 263. Auflage. Walter de Gruyter, Berlin 2012

Raz N, Lindenberger U, Rodrigue KM, Kennedy KM, Head D, Williamson A, Dahle C, Gerstorf D, Acker JD (2005): Regional Brain Changes in Aging Healthy Adults: General Trends, Individual Differences and Modifiers. Cerebral Cortex 15(11), 1676-1689

Ropele S, Filippi M, Valsasina P, Korteweg T, Barkhof F, Tofts PS, Samson R, Miller DH, Fazekas F (2005): Assessment and correction of B1-induced errors in magnetization transfer ratio measurements. Magn Reson Med 53(1), 134-140

Schaltenbrand G, Wahren W: Atlas for stereotaxy of the human brain with an accompanying guide. 2nd Edition; Thieme, Stuttgart 1977

Schulz-Schaeffer WJ, Gringel T, Helms G: Private communication in an informal meeting to discuss the delineation of the thalamus. MR-Forschung in der Neurologie und Psychiatrie, Göttingen, 22 October 2008

Sherman SM, Guillery RW: Exploring the thalamus. Academic Press, San Diego 2001

Sherman SM (2005): Thalamic relays and cortical functioning. Prog Brain Res 149, 107-126

Sherman SM (2007): The thalamus is more than just a relay. Curr Opin Neurobiol 17(4), 417422

Spatz H (1922): Über den Eisennachweis im Gehirn, besonders in Zentren des extrapyramidalmotorischen Systems. Z Gesamte Neurol Psychiatry 77(1), 261-390

Symms M, Jäger HR, Schmierer K, Yousry TA (2004): A review of structural magnetic resonance neuroimaging. J Neurol Neurosurg Psychiatry 75(9), 1235-1244

Talairach J: Atlas d'anatomie stéréotaxique repérage radiologique indirect des noyaux gris centraux des régions mésencéphalo-sous-optique et hypothalamique de l'homme. Masson, Paris 1957

Toga AW, Thompson PM, Mori S, Amunts K, Zilles K (2006): Towards multimodal atlases of the human brain. Nat Rev Neurosci 7(12), 952-966

Toncray JE, Krieg WJS (1946): The nuclei of the human thalamus: A comparative approach. J Comp Neurol $\underline{85}(3), 421-459$

Unrath A, Klose U, Grodd W, Ludolph AC, Kassubek J (2008): Directional encoding of the human thalamus by diffusion tensor imaging. Neurosci Lett 434(3), 322-327

van Buren JM, Borke RC: Variations and connections of the human thalamus. 2 Volumes. Springer Verlag, Berlin 1972

Vandewalle V, van der Linden C, Groenewegen HJ, Caemaert J (1999): Stereotactic treatment of Gilles de la Tourette syndrome by high frequency stimulation of thalamus. Lancet $\underline{353}(9154), 724$

Vogt O, Vogt C (1941): Thalamusstudien I-III. J Psychol Neurol 50(1-2), 33-154

Walker AE: The Primate Thalamus. The University of Chicago Press, Chicago, Illinois 1938

Welter M-L, Mallet L, Houeto J-L, Karachi C, Czernecki V, Cornu P, Navarro S, Pidoux B, Dormont D, Bardinet E, et al. (2008): Internal Pallidal and Thalamic Stimulation in Patients with Tourette Syndrome. Arch Neurol 65(7), 952-957

Wiegell MR, Tuch DS, Larsson HB, Wedeen VJ (2003): Automatic segmentation of thalamic nuclei from diffusion tensor magnetic resonance imaging. Neuroimage $\underline{19}(2 \mathrm{Pt} 1), 391$ 401 
Wilke M, Turchi J, Smith K, Mishkin M, Leopold DA (2010): Pulvinar Inactivation Disrupts Selection of Movement Plans. J Neurosci 30(25), 8650-8659

Wolff SD, Balaban RS (1989): Magnetization transfer contrast (MTC) and tissue water proton relaxation in vivo. Magn Reson Med 10(1), 135-144

Yagishita A, Nakano I, Oda M, Hirano A (1994): Location of the corticospinal tract in the internal capsule at MR imaging. Radiology 191(2), 455-460

Yamamoto T, Katayama Y, Obuchi T, Kano T, Kobayashi K, Oshima H, Fukaya C (2006): Thalamic sensory relay nucleus stimulation for the treatment of peripheral deafferentation pain. Stereotact Funct Neurosurg 84(4), 180-183

Yelnik J, Bardinet E, Dormont D, Malandain G, Ourselin S, Tande D, Karachi C, Ayache N, Cornu P, Agid Y (2007): A three-dimensional, histological and deformable atlas of the human basal ganglia. I. Atlas construction based on immunohistochemical and MRI data. Neuroimage 34(2), 618-638

Ziyan U, Tuch DS, Westin C (2006): Segmentation of thalamic nuclei from DTI using spectral clustering. Med Image Comput Comput Assist Interv 9(Pt 2), 807-814

Ziyan U, Westin C (2008): Joint segmentation of thalamic nuclei from a population of diffusion tensor MR images. Med Image Comput Comput Assist Interv 11(Pt 1), 279-286

Zrinzo L, Zrinzo LV, Tisch S, Limousin PD, Yousry TA, Afshar F, Hariz MI (2008): Stereotactic localization of the human pedunculopontine nucleus: atlas-based coordinates and validation of a magnetic resonance imaging protocol for direct localization. Brain $\underline{131}(6), 1588-1598$ 


\section{DANKSAgung}

An dieser Stelle möchte ich danke sagen an all diejenigen, die mich während meiner Doktorarbeit begleitet und unterstützt haben.

$\mathrm{Zu}$ allererst gilt mein besonderer Dank meinem Doktorvater und Betreuer PD Dr. Gunther Helms für seine ausdauernde Unterstützung, seine unermüdliche Bereitschaft, komplexe physikalische Zusammenhänge zu erläutern, sowie die hervorragende Betreuung und Anleitung.

Ein großes Dankeschön geht an die Abteilung Kognitive Neurologie und die MRForschergruppe in der Neurologie und Psychiatrie, insbesondere an Prof. Dr. Melanie Wilke und PD Dr. Peter Dechent, für die Arbeitsmöglichkeit am MRI-Scanner und die Möglichkeit, erste Arbeitsabläufe in der wissenschaftlichen Forschung kennen zu lernen.

Weiterer besonderer Dank gilt ferner PD Dr. Walter Schulz-Schaeffer für den stets wertvollen, konstruktiven und auch zeitintensiven fachlichen Austausch über die Anatomie der Thalamuskerne.

Danke sagen möchte ich Prof. Dr. Michael Knauth für die vertrauensvolle Bereitstellung seines Schaltenbrand-und-Wahren-Atlas und die Möglichkeit des Vortragens auf dem 88. Deutschen Röntgenkongress 2007 in Berlin.

Bedanken möchte ich mich auch bei Dr. Andreas Frölich für die ROI-Analyse, welche in die Publikation eingegangen ist.

Abschließend möchte ich mich bei allen Probanden bedanken, dass sie sich zur Verfügung gestellt und so ruhig während der Messungen ausgehalten haben.

Vielen Dank! 